MATHEMATICS OF COMPUTATION

Volume 75, Number 253, Pages 7-41

S 0025-5718(05)01802-8

Article electronically published on October 12, 2005

\title{
FINITE ELEMENT APPROXIMATION OF A PHASE FIELD MODEL FOR SURFACE DIFFUSION OF VOIDS IN A STRESSED SOLID
}

\author{
JOHN W. BARRETT, HARALD GARCKE, AND ROBERT NÜRNBERG
}

\begin{abstract}
We consider a fully practical finite element approximation of the degenerate Cahn-Hilliard equation with elasticity: Find the conserved order parameter, $\theta(x, t) \in[-1,1]$, and the displacement field, $\underline{u}(x, t) \in \mathbb{R}^{2}$, such that$$
\gamma \frac{\partial \theta}{\partial t}=\nabla \cdot\left(b(\theta) \nabla\left[-\gamma \Delta \theta+\gamma^{-1} \Psi^{\prime}(\theta)+\frac{1}{2} c^{\prime}(\theta) \mathcal{C} \underline{\underline{\mathcal{E}}}(\underline{u}): \underline{\underline{\mathcal{E}}}(\underline{u})\right]\right),
$$$$
\nabla \cdot(c(\theta) \mathcal{C} \underline{\underline{\mathcal{E}}}(\underline{u}))=\underline{0},
$$

subject to an initial condition $\theta^{0}(\cdot) \in[-1,1]$ on $\theta$ and boundary conditions on both equations. Here $\gamma \in \mathbb{R}_{>0}$ is the interfacial parameter, $\Psi$ is a nonsmooth double well potential, $\underline{\underline{\mathcal{E}}}$ is the symmetric strain tensor, $\mathcal{C}$ is the possibly anisotropic elasticity tensor, $c(s):=c_{0}+\frac{1}{2}\left(1-c_{0}\right)(1+s)$ with $c_{0}(\gamma) \in \mathbb{R}_{>0}$ and $b(s):=1-s^{2}$ is the degenerate diffusional mobility. In addition to showing stability bounds for our approximation, we prove convergence, and hence existence of a solution to this nonlinear degenerate parabolic system in two space dimensions. Finally, some numerical experiments are presented.

\section{INTRODUCTION}

Integrated circuits contain thin metallic lines (interconnects) that make electrical contact between different components of the device. These lines are passivated with a layer of oxide at large temperatures, and during the cooling process large stresses are induced. Also voids nucleate in the interconnect, and they migrate and change their shape due to the diffusion of atoms. One of the major failure mechanisms in modern micro-electronic circuits is that voids cut the whole interconnect and cause an open circuit. The understanding of how voids migrate is therefore of great practical interest.

In general, diffusion in the bulk of the interconnect is much slower than that on the surface of the void. Therefore we will restrict ourselves to the case where diffusion is restricted to the surface of the void or more precisely to a diffuse layer at the void surface. In this case there are three main driving forces for diffusion: one results from capillary effects and the other two are due to electromigration and stressmigration. To formulate the latter two we need to introduce the electric potential $\phi$, the displacement field $\underline{u}$, the symmetric strain tensor $\underline{\mathcal{E}}(\underline{u}):=\frac{1}{2}\left(\nabla \underline{u}+(\nabla \underline{u})^{T}\right)$ and the elastic energy density $E(\underline{u}):=\frac{1}{2} \mathcal{C} \underline{\underline{\mathcal{E}}}(\underline{u}): \underline{\underline{\mathcal{E}}}(\underline{u})$. Here $\mathcal{C}$ is the possibly anisotropic

Received by the editor April 21, 2004 and, in revised form, January 26, 2005.

2000 Mathematics Subject Classification. Primary 65M60, 65M12, 65M50, 35K55, 35K65, $35 \mathrm{~K} 35,82 \mathrm{C} 26,74 \mathrm{~F} 15$. 
elasticity tensor, which we assume to be symmetric and positive definite. The product $\underline{\underline{\mathcal{A}}}: \underline{\underline{\mathcal{B}}}$ of two matrices $\underline{\underline{\mathcal{A}}}, \underline{\underline{\mathcal{B}}} \in \mathbb{R}^{d \times d}$ is defined as $\sum_{i, j=1}^{d} \mathcal{A}_{i j} \mathcal{B}_{i j}$. We denote by $V$ the normal velocity of the void surface, $\Gamma(t)$, with the normal pointing into the void and by $\kappa$ its mean curvature with the sign convention that $\kappa$ is positive if the interface is curved in the direction of the normal. Then mass conservation gives

$$
V=-\nabla_{s} \cdot \underline{J}_{s} \quad \text { on } \Gamma(t), \quad \text { where } \quad \underline{J}_{s}=-D_{s} \nabla_{s}(-\varsigma \kappa+E(\underline{u})+\alpha \phi)
$$

is the mass flux, $\nabla_{s}$. is the surface divergence, $\nabla_{s}$ is the surface gradient, $D_{s}$ is a constant related to the surface diffusivity and $\varsigma$ is the surface energy density. Here the first term describes capillary forces, the second describes forces resulting from changes in the elastic energy and the forcing term $\alpha \nabla_{s} \phi$ is caused by an electric current in the bulk of the material and this force is related to the "electron wind" force. The above equations for the surface motion then have to be coupled to the Laplace equation for the electric potential $\phi$, the quasi-static mechanical equilibrium equations for $\underline{u}$ and appropriate boundary conditions. For more details we refer to [46], [16] and [36].

Let us briefly discuss the influence of the three terms of the mass flux in (1.1). The first term leads to diffusion of atoms from regions of small mean curvature to regions of high mean curvature. If only capillary effects were present the length/area of the void surface would decrease and the voids would become circular/spherical; see 23 and 24. The second term leads to diffusion from regions of high elastic energy to regions of smaller elastic energy, and the third gives rise to diffusion in the direction opposite to the electric field (this is true if $\alpha<0$ and this is the case for aluminum, which is mainly used for interconnects). The effect of all three terms can be seen in numerical simulations; see, e.g., [14] and [12, §5]. From these numerical simulations one notices that the topology of the voids can change. Therefore numerical methods that depend on the direct parametrization of the void surface will have difficulties. For an overview on numerical methods for interface motion and their advantages and disadvantages we refer to [21].

In this paper we study a finite element approximation of a phase field model for surface diffusion of voids due to capillary effects and stressmigration. We will not include electromigration since a phase field method for surface diffusion in the presence of electromigration (and in the absence of stressmigration) was already analysed in 12. A phase field model for electromigration of intergranular voids, i.e., of voids in solids with different grain orientations, will be discussed in [8]. Furthermore, we will present numerical simulations of the combined effect of surface diffusion, electromigration and stressmigration in a forthcoming paper where we will also discuss applications to epitaxial growth; see 9 .

In a phase field model a diffuse layer is used to describe interfaces or free surfaces. To model surface diffusion by a phase field model we introduce an order parameter $\theta$ which (away from a small interfacial layer) attains the value -1 in the void and the value 1 in regions occupied by the metal. In the diffuse interfacial layer $\theta$ varies continuously from -1 to 1 . The free energy for the evolution law (1.1) is given by

$$
\int_{\Gamma(t)} \varsigma \mathrm{d} s+\int_{\Omega_{+}(t)} E(\underline{u}) \mathrm{d} x
$$

where the first term is the integral of the surface energy density $\varsigma$ over the void surface and $\Omega_{+}$is the region occupied by the metal. In phase field models the 
surface energy density $\varsigma$ is now replaced by a Ginzburg-Landau free energy density $\varsigma \frac{2}{\pi}\left[\frac{\gamma}{2}|\nabla \theta|^{2}+\gamma^{-1} \Psi(\theta)\right]$, where $\gamma$ is a small positive parameter related to the interfacial thickness and $\Psi$ is a free energy density with two global minima at \pm 1 . In the above, and throughout, we will use for convenience an obstacle free energy of the form

$$
\Psi(s):=\left\{\begin{array}{lll}
\frac{1}{2}\left(1-s^{2}\right) & \text { if } & s \in[-1,1] \\
\infty & \text { if } s \notin[-1,1]
\end{array}\right.
$$

which restricts the order parameter $\theta$ to lie in the interval $[-1,1]$ and also guarantees that outside a small interfacial layer, $\theta$ attains the values \pm 1 ; see, e.g., 15 .

The elastic energy density also has to take the interfacial layer into account and is hence given by

$$
E(\theta, \underline{u}):=\frac{1}{2} c(\theta) \mathcal{C} \underline{\underline{\mathcal{E}}}(\underline{u}): \underline{\underline{\mathcal{E}}}(\underline{u}),
$$

where $c$ is an interpolation function given by

$$
c(s):=c_{0}+\frac{1}{2}\left(1-c_{0}\right)(1+s) .
$$

Here $c_{0}=c_{0}(\gamma) \in(0,1)$ is small, and we will assume that $c_{0}(\gamma) \rightarrow 0$ as $\gamma \rightarrow 0$. Hence, $c$ is affine linear with $c(-1)=c_{0} \leq c(s) \leq 1=c(1)$ for all $s \in[-1,1]$. Now the total free energy for the phase field model is given by

$$
J(\theta, \underline{u}):=\int_{\Omega}\left[\varsigma \frac{2}{\pi}\left\{\frac{\gamma}{2}|\nabla \theta|^{2}+\gamma^{-1} \Psi(\theta)\right\}+E(\theta, \underline{u})\right] \mathrm{d} x
$$

with the possible addition of an integral over the boundary of $\Omega$, depending on the imposed boundary conditions on $\underline{u}$.

Now we define the chemical potential, $w$, via the first variation of $J$ with respect to $\theta$ :

$$
\left.w=\frac{\delta J}{\delta \theta}=\left[\varsigma \frac{2}{\pi}\left(-\gamma \Delta \theta+\gamma^{-1} \Psi^{\prime}(\theta)\right)+\frac{1}{2} c^{\prime}(\theta) \mathcal{C} \underline{\underline{\mathcal{E}}} \underline{u}\right): \underline{\underline{\mathcal{E}}}(\underline{u})\right],
$$

which is the diffusion potential for $\theta$. The diffusion equation for $\theta$ is then given by

$$
\gamma \frac{\partial \theta}{\partial t}=\nabla \cdot\left(\frac{8}{\pi} D_{s} b(\theta) \nabla w\right),
$$

where

$$
b(s):=1-s^{2} \quad \forall s \in[-1,1]
$$

is a degenerate mobility that is zero outside of the interfacial layer. Hence diffusion is restricted to the interfacial layer, which is conceptually close to the idea of surface diffusion where diffusion only takes place on the surface. In fact it was shown in [18, using formally matched asymptotic expansions, that (in the absence of elastic effects) the phase field equations as stated above converge, as the interfacial parameter $\gamma \rightarrow 0$, to motion by surface diffusion.

If we include elasticity and require quasi-static equilibrium, i.e.,

$$
\nabla \cdot(c(\theta) \mathcal{C} \underline{\underline{\mathcal{E}}}(\underline{u}))=\underline{0},
$$

we obtain in the limit $\gamma \rightarrow 0$ and $c_{0}(\gamma) \rightarrow 0$ that the zero level sets of $\theta$ converge to a hypersurface $\Gamma(t)$ that evolves according to the law

$$
V=D_{s} \Delta_{s}\left[-\varsigma \kappa+\frac{1}{2} \mathcal{C} \underline{\underline{\mathcal{E}}}(\underline{u}): \underline{\underline{\mathcal{E}}}(\underline{u})\right] \quad \text { on } \Gamma(t) .
$$

This can be shown using formally matched asymptotic expansions when one combines the approaches of [18, 39] and [25]. 
The system (1.5)-(1.8) is a degenerate Cahn-Hilliard equation coupled to an elasticity system. If $\mathcal{C} \equiv 0$, then (1.5)-(1.8) collapses to the degenerate CahnHilliard equation without elasticity. Existence of a solution to this fourth-order degenerate parabolic equation for $\theta$, as $b(\theta)$ can take on zero values, can be found in 22. Degenerate parabolic equations of higher order exhibit some new characteristic features which are fundamentally different to those for second-order degenerate parabolic equations. The key point is that there is no maximum or comparison principle for parabolic equations of higher order. This drastically complicates the analysis since a lot of results which are known for second-order equations are proven with the help of comparison techniques. Related to this is the fact that there is no uniqueness result known for (1.5)-(1.7) with $\mathcal{C} \equiv 0$. Although there is no comparison principle, one of the main features of this system is the fact that one can show existence of a solution with $|\theta| \leq 1$ if given initial data $\left|\theta^{0}\right| \leq 1$. This is in contrast to linear parabolic equations of fourth order.

In the case of a constant mobility, i.e., $b(\theta) \equiv 1$, the system (1.5), (1.6) and (1.8) was studied analytically in [26], 27] and [19]. For a finite element approximation in this nondegenerate case; see, e.g., 29] and [30.

There is very little work on the numerical analysis of degenerate parabolic equations of fourth order: for work on the thin film equation, see [4, 47] and 35]; for thin film flows in the presence of surfactants, see 7; and for work on degenerate Cahn-Hilliard systems, see [5, [6] and [3]. In all of these papers, although stability bounds were proved in one and two space dimensions, the main convergence result was restricted to one space dimension. However, convergence in two space dimensions of a finite element approximation to the thin film equation has been recently proved in [34. This approach was extended in (i) [1] and (ii) [12] to prove convergence in two space dimensions of a finite element approximation to (i) the thin film equation in the presence of surfactants and repulsive van der Waals forces, and (ii) the phase field approximation of (1.1) in the absence of stressmigration. It is the aim of this paper to propose and prove convergence of a finite element approximation of the degenerate system (1.5)-(1.8) and hence prove existence of a solution to (1.5)-(1.8). Since in the stressmigration case a term that is quadratic in the gradient of $\underline{u}$ - as opposed to linear in $\phi$ in the electromigration case - appears in the chemical potential $w$ (see (1.5)), this makes the convergence analysis in the presence of stressmigration far more complicated than that of electromigration.

Due to a lack of embedding properties, our convergence analysis is restricted to two spatial dimensions (i.e., $d=2$ ). For ease of exposition, we will restrict our presentation throughout to this case. However, the phase field approach and the corresponding finite element approximation with the basic energy bound (see (2.67a) below) are easily extended to three spatial dimensions. We adopt the following notation throughout. The trace of a tensor $\underline{\underline{\mathcal{A}}}$ is denoted by $\operatorname{Tr}(\underline{\underline{\mathcal{A}}}):=\mathcal{A}_{11}+\mathcal{A}_{22}$, and the divergence is defined as $\nabla \cdot \underline{\underline{\mathcal{A}}}=\left(\frac{\partial \mathcal{A}_{11}}{\partial x_{1}}+\frac{\partial \mathcal{A}_{12}}{\partial x_{2}}, \frac{\partial \mathcal{A}_{21}}{\partial x_{1}}+\frac{\partial \mathcal{A}_{22}}{\partial x_{2}}\right)^{T}$; see, e.g., [17, Chapter 11]. We will assume throughout for all $i, j, k, l \in\{1,2\}$ that

$$
\text { (i) } \quad \mathcal{C}_{i j k l}=\mathcal{C}_{j i k l}=\mathcal{C}_{i j l k} \quad \text { and } \quad \text { (ii) } \quad \mathcal{C}_{i j k l}=\mathcal{C}_{k l i j}
$$

Here (i) follows, without loss of generality, from the fact that $\mathcal{C}$ maps symmetric tensors to symmetric tensors; and (ii) follows from the symmetry assumption $\mathcal{C} \underline{\underline{\mathcal{A}}}$ : $\underline{\underline{\mathcal{B}}}=\underline{\underline{\mathcal{A}}}: \mathcal{C} \underline{\underline{\mathcal{B}}}$. We assume also throughout that $\mathcal{C}$ is positive definite; that is, there 
exist constants $m_{\mathcal{C}}, M_{\mathcal{C}}>0$ such that

$$
0<m_{\mathcal{C}}(\underline{\underline{\mathcal{A}}}: \underline{\underline{\mathcal{A}}}) \leq \mathcal{C} \underline{\underline{\mathcal{A}}}: \underline{\underline{\mathcal{A}}} \leq M_{\mathcal{C}}(\underline{\underline{\mathcal{A}}}: \underline{\underline{\mathcal{A}}}) \quad \forall \underline{\underline{\mathcal{A}}} \in \mathbb{R}^{2 \times 2} \backslash\{\underline{\underline{0}}\} .
$$

If one further assumes cubic symmetry, then it also follows that $\mathcal{C}_{1111}=\mathcal{C}_{2222}$ and $\mathcal{C}_{2212}=\mathcal{C}_{1112}=0$; see, e.g., 37. For an isotropic material we obtain that

$$
\mathcal{C} \underline{\underline{\mathcal{E}}}(\underline{u})=2 \mu \underline{\underline{\mathcal{E}}}(\underline{u})+\lambda \operatorname{Tr}(\underline{\underline{\mathcal{E}}}(\underline{u})) \underline{\underline{\mathcal{I}}},
$$

where $\underline{\underline{\mathcal{I}}}$ is the identity tensor, and $\mu \in \mathbb{R}_{>0}$ and $\lambda \in \mathbb{R}_{\geq 0}$ are the Lamé moduli. In what follows, to simplify the presentation, we will set, without loss of generality, the surface diffusivity $D_{s}=\frac{\pi}{8}$ and the surface energy density $\varsigma=\frac{\pi}{2}$.

In the following we will analyse a finite element approximation of the nonlinear degenerate parabolic system for a given $\gamma \in \mathbb{R}_{>0}$ :

(P) Find functions $\theta: \Omega \times[0, T] \rightarrow[-1,1], w: \Omega \times[0, T] \rightarrow \mathbb{R}$ and $\underline{u}: \Omega \times[0, T] \rightarrow$ $\mathbb{R}^{2}$ such that

$$
\begin{aligned}
& \gamma \frac{\partial \theta}{\partial t}=\nabla \cdot(b(\theta) \nabla w) \quad \text { in } \Omega_{T}, \\
& w=-\gamma \Delta \theta+\gamma^{-1} \Psi^{\prime}(\theta)+\frac{1}{2} c^{\prime}(\theta) \mathcal{C} \underline{\underline{E}}(\underline{u}): \underline{\underline{\mathcal{E}}}(\underline{u}) \text { on }\{|\theta|<1\}, \\
& \nabla \theta \cdot \underline{\nu}=b(\theta) \nabla w \cdot \underline{\nu}=0 \quad \text { on } \partial \Omega \times(0, T], \\
& \theta(x, 0)=\theta^{0}(x) \in[-1,1] \quad \forall x \in \Omega, \\
& \nabla \cdot(c(\theta) \mathcal{C} \underline{\underline{\mathcal{E}}}(\underline{u}))=\underline{0} \quad \text { in } \Omega_{T}, \quad c(\theta) \mathcal{C} \underline{\underline{\mathcal{E}}}(\underline{u}) \underline{\nu}=\underline{g} \quad \text { on } \partial \Omega \times(0, T],
\end{aligned}
$$

where $\Omega$ is a Lipschitz domain in $\mathbb{R}^{2}$ with $\underline{\nu}$ the outward unit normal to its boundary $\partial \Omega, T>0$ is a fixed positive time, and $\Omega_{T}:=\Omega \times(0, T]$. The function $\underline{g} \in \underline{L}^{2}(\partial \Omega)$ is the given boundary force satisfying the necessary compatibility conditions, $\int_{\partial \Omega} \underline{g} \mathrm{~d} s=\underline{0}$ and $\int_{\partial \Omega} \underline{g} \cdot\left(x_{2},-x_{1}\right)^{T} \mathrm{~d} s=0$, for the existence of a solution $\underline{u}$ to $(1.12 \mathrm{e})$. For simplicity, we will consider

$$
\underline{g}=\underline{\underline{S}} \underline{\nu}=\mathcal{C} \underline{\underline{S}}^{*} \underline{\nu}
$$

where $\underline{\underline{S}} \in \mathbb{R}^{2 \times 2}$ is a symmetric tensor and $\underline{\underline{S}}^{*}:=\mathcal{C}^{-1} \underline{\underline{S}}$. Alternatively, one could prescribe displacement boundary conditions, $\underline{u}=\underline{f}$, on $\bar{\partial} \Omega$ or on parts thereof.

We should note that the solution $\underline{u}$ to (1.12e) is not unique. This is simply because

$$
\underline{\underline{\mathcal{E}}}(\underline{v})=\underline{0} \quad \forall \underline{v} \in \underline{\mathrm{RM}},
$$

where $\underline{\mathrm{RM}}$ is the space of rigid motions and characterized by $\underline{\mathrm{RM}}:=\left\{\underline{v} \in \underline{H}^{1}(\Omega)\right.$ : $\left.\underline{v}=\underline{p}+q\left(x_{2},-x_{1}\right)^{T}, \underline{p} \in \mathbb{R}^{2}, q \in \mathbb{R}\right\}$. Hence one can impose uniqueness for (1.12e) by seeking $\underline{u}$ such that $\int_{\Omega} \underline{u} \cdot \underline{v} \mathrm{~d} x=0$ for all $\underline{v} \in \underline{\mathrm{RM}}$; see our definition of $\underline{\hat{V}}_{p}$ in (1.18) below.

The basic ingredients of our approach are some key energy estimates. First, we relate $G$ to $b$ by the identity $b(s) G^{\prime \prime}(s) \equiv 1$. Knowing $b$, recall (1.7), this identity determines $G$ up to a linear term. Furthermore we have that $G$ is convex. One can then derive formally the following energy estimates for (P). Testing (1.12a with $w$ and (1.12b) with $\frac{\partial \theta}{\partial t}$, combining and noting (1.12c, e) and (1.3) yields that $(1.15)$

$\frac{\mathrm{d}}{\mathrm{d} t}\left[\int_{\Omega}\left[\frac{1}{2} \gamma|\nabla \theta|^{2}+\gamma^{-1} \Psi(\theta)+E(\theta, \underline{u})\right] \mathrm{d} x-\int_{\partial \Omega} \underline{g} \cdot \underline{u} \mathrm{~d} s\right]+\gamma^{-1} \int_{\Omega} b(\theta)|\nabla w|^{2} \mathrm{~d} x \leq 0$. 
Testing (1.12a with $G^{\prime}(\theta)$ and (1.12b) with $-\Delta \theta$, combining and noting (1.2), (1.10) and (1.4) yields that

$$
\gamma^{2} \frac{\mathrm{d}}{\mathrm{d} t} \int_{\Omega} G(\theta) \mathrm{d} x+\frac{1}{2} \gamma^{2} \int_{\Omega}|\Delta \theta|^{2} \mathrm{~d} x \leq \int_{\Omega}|\nabla \theta|^{2} \mathrm{~d} x+\frac{1}{32} M_{\mathcal{C}}^{2} \int_{\Omega}|\underline{\underline{\mathcal{E}}}(\underline{u})|^{4} \mathrm{~d} x .
$$

In order to bound $\Delta \theta$ in $L^{2}\left(\Omega_{T}\right)$, one needs to bound $\nabla \underline{u}$ in $L^{4}\left(\Omega_{T}\right)$. This is the key difficulty when including the elastic effects. This is achieved by using an $L^{\infty}\left(0, T ; \underline{W}^{1, p}(\Omega)\right), p>2$, bound for $\underline{u}$ solving (1.12e) which does not depend on the choice of $\theta \in L^{\infty}\left(\Omega_{T}\right)$; see [26], 28] and Lemma 1.1] together with Remark 2.2 below.

It is the goal of this paper to derive a finite element approximation of $(\mathrm{P})$ that is consistent with the energy estimates (1.15) and (1.16). In order to derive a discrete analogue of the energy estimate (1.16), we adapt a technique introduced in [47] and 35. for deriving a discrete entropy bound for the thin film equation; see also [12. However, the key difficulty here in proving convergence of our finite element approximation, and hence existence of a solution to $(\mathrm{P})$, is the finite element analogue of the crucial $\underline{W}^{1, p}(\Omega), p>2$, bound for $\underline{u}$; see Lemma 2.3 below.

This paper is organised as follows. In $\$ 2$ we formulate a fully practical finite element approximation of the degenerate system $(\mathrm{P})$ and derive discrete analogues of the energy estimates (1.15) and (1.16). In $\$ 3$ we prove convergence, and hence existence of a solution to the system (P) in two space dimensions. Finally, in 84 we present some numerical experiments.

Notation and auxiliary results. Throughout this paper we will make use of the standard notation for Sobolev spaces, their norms and semi-norms; see, e.g., 12, $\S 1]$. In addition $(\cdot, \cdot)$ denotes the standard $L^{2}$ inner product over $\Omega$. Furthermore we define $f \eta:=\frac{1}{\underline{m}(\Omega)}(\eta, 1)$ for all $\eta \in L^{1}(\Omega)$.

For later purposes, we recall the following well-known Sobolev interpolation result, e.g., see [1]: Let $q \in(1, \infty), r \in[q, \infty)$ if $q \geq 2$ and $r \in\left[q, \frac{2 q}{2-q}\right]$ if $q \in(1,2)$; and $\mu:=\frac{2}{q}-\frac{2}{r}$. Then the following inequality holds:

$$
|z|_{0, r} \leq C|z|_{0, q}^{1-\mu}\|z\|_{1, q}^{\mu} \quad \forall z \in W^{1, q}(\Omega) .
$$

For $p \in[1, \infty]$, we introduce also

$$
\underline{\widehat{V}}_{p}:=\left\{\underline{\eta} \in \underline{W}^{1, p}(\Omega):(\underline{\eta}, \underline{v})=0 \quad \forall \underline{v} \in \underline{\mathrm{RM}}\right\}
$$

and define $\underline{\widehat{H}}^{1}(\Omega):=\widehat{\widehat{V}}_{2}$. We recall the following version of Korn's inequality:

$$
\|\underline{\eta}\|_{1, p} \leq C|\underline{\underline{\mathcal{E}}}(\underline{\eta})|_{0, p} \quad \forall \underline{\eta} \in \underline{\widehat{V}}_{p}, \quad p \in(1, \infty) ;
$$

see, e.g., [41, p. 79] for the case $p=2$, or [40] for general $p \in(1, \infty)$. Furthermore, the following lemma holds.

Lemma 1.1. There exists a $\delta \in \mathbb{R}_{>0}$ such that for all $p \in\left[\frac{2+\delta}{1+\delta}, 2+\delta\right]$ there is a $\beta(p) \geq 1$ satisfying

$$
|\underline{\underline{\mathcal{E}}}(\underline{z})|_{0, p} \leq \beta(p) \sup _{\underline{0} \neq \underline{\underline{\eta}} \in \underline{\underline{\underline{V}}}_{q}} \frac{(\underline{\underline{\mathcal{E}}}(\underline{z}), \underline{\underline{\mathcal{E}}}(\underline{\eta}))}{|\underline{\underline{\mathcal{E}}}(\underline{\eta})|_{0, q}} \quad \forall \underline{z} \in \underline{\widehat{V}}_{p}
$$

where $\frac{1}{p}+\frac{1}{q}=1$. Moreover $\beta$ is continuous on the interval $\left[\frac{2+\delta}{1+\delta}, 2+\delta\right]$ and $\beta(p) \rightarrow$ $\beta(2)=1$ as $p \rightarrow 2$. 
Proof. Let $\left[L^{p}(\Omega)\right]_{\mathrm{sym}}^{2 \times 2}:=\left\{\underline{\underline{\mathcal{F}}} \in\left[L^{p}(\Omega)\right]^{2 \times 2}: \underline{\underline{\mathcal{F}}}\right.$ is symmetric $\}$. For $\underline{z} \in \underline{\widehat{V}}_{p}$ we define $\mathcal{S}(\underline{\underline{\mathcal{F}}}):=(\underline{\underline{\mathcal{E}}}(\underline{z}), \underline{\underline{\mathcal{F}}})$ for all $\underline{\underline{\mathcal{F}}}=\underline{\underline{\mathcal{E}}}(\underline{\eta})$ with $\underline{\underline{\eta}} \in \underline{\underline{V}}_{q}$. $\mathcal{S}$ is a continuous linear functional on a closed subspace of $\left[L^{q}(\Omega)\right]_{\mathrm{sym}}^{2 \times 2}$ with

$$
\|\mathcal{S}\|=\sup _{\underline{0} \neq \underline{\underline{\eta}} \underline{\widehat{V}_{q}}} \frac{(\underline{\underline{\mathcal{E}}}(\underline{z}), \underline{\underline{\mathcal{E}}}(\underline{\eta}))}{|\underline{\underline{\mathcal{E}}}(\underline{\eta})|_{0, q}}
$$

The Hahn-Banach theorem and the fact that $\left(\left[L^{q}(\Omega)\right]_{\mathrm{sym}}^{2 \times 2}\right)^{\prime} \cong\left[L^{p}(\Omega)\right]_{\mathrm{sym}}^{2 \times 2}$ imply the existence of a $\underline{\underline{\mathcal{G}_{z}}} \in\left[L^{p}(\Omega)\right]_{\mathrm{sym}}^{2 \times 2}$ such that

$$
(\underline{\underline{\mathcal{E}}}(\underline{z}), \underline{\underline{\mathcal{F}}})=\left(\underline{\underline{\mathcal{G}_{z}}}, \underline{\underline{\mathcal{F}}}\right) \quad \forall \underline{\underline{\mathcal{F}}} \in\left[L^{q}(\Omega)\right]_{\mathrm{sym}}^{2 \times 2} \quad \text { and } \quad\left|\underline{\underline{\mathcal{G}_{z}}}\right|_{0, p}=\sup _{\underline{0} \neq \underline{\underline{\eta}} \in \underline{\underline{\underline{V}}}_{q}} \frac{(\underline{\underline{\mathcal{E}}}(\underline{z}), \underline{\underline{\mathcal{E}}}(\underline{\eta}))}{|\underline{\underline{\mathcal{E}}}(\underline{\eta})|_{0, q}} .
$$

Let $\mathcal{Q}:\left[L^{p}(\Omega)\right]_{\mathrm{sym}}^{2 \times 2} \rightarrow\left[L^{p}(\Omega)\right]_{\mathrm{sym}}^{2 \times 2}$ be the linear operator such that $\mathcal{Q} \underline{\underline{\mathcal{F}}}=\underline{\underline{\mathcal{E}}}\left(\underline{f}_{\mathcal{F}}\right)$, where $\underline{f}_{\mathcal{F}} \in \widehat{\underline{V}}_{p}$ is such that

$$
\left(\underline{\underline{\mathcal{E}}}\left(\underline{f}_{\mathcal{F}}\right), \underline{\underline{\mathcal{E}}}(\underline{\eta})\right)=(\underline{\underline{\mathcal{F}}}, \underline{\underline{\mathcal{E}}}(\underline{\eta})) \quad \forall \underline{\eta} \in \underline{\widehat{V}}_{q} .
$$

We need to show that $\mathcal{Q}$ is well-defined and compute the operator norm $\|\mathcal{Q}\|_{p}$ of $\mathcal{Q}$. The well-posedness of $\mathcal{Q}$ for $p=2$ follows from (1.19) and the Lax-Milgram theorem; and in addition, $\|\mathcal{Q}\|_{2}=1$. Moreover, regularity theory implies that there exists a $\delta>0$ such that for all $p \in[2,2+\delta]$ if $\underline{\underline{\mathcal{F}}} \in\left[L^{p}(\Omega)\right]_{\mathrm{sym}}^{2 \times 2}$, then it follows that

$$
|\mathcal{Q} \underline{\underline{\mathcal{F}}}|_{0, p} \leq C(p)\left[\left|\nabla \underline{f}_{\mathcal{F}}\right|_{0,2}+|\underline{\underline{\mathcal{F}}}|_{0, p}\right] \leq C(p)\left[|\underline{\underline{\mathcal{F}}}|_{0,2}+|\underline{\underline{\mathcal{F}}}|_{0, p}\right] \leq C(p)|\underline{\underline{\mathcal{F}}}|_{0, p} .
$$

The first inequality in the above can be shown for example with the help of a method introduced in 31, which proved local $L^{p}$-estimates for gradients of solutions to elliptic systems. In [26 and 28 this method has been applied to obtain global $L^{p}$-estimates for gradients of solutions to elasticity systems on Lipschitz domains. The above shows that $\mathcal{Q}$ is a bounded linear operator for $p \in[2,2+\delta]$ and that $\|\mathcal{Q}\|_{p} \leq C(p)$.

We now want to show that $\mathcal{Q}$ is also a linear continuous operator on $\left[L^{q}(\Omega)\right]_{\mathrm{sym}}^{2 \times 2}$, where $q$ is such that $\frac{1}{p}+\frac{1}{q}=1$ for a $p \in[2,2+\delta]$. To do so, we approximate $\underline{\underline{\mathcal{F}}} \in\left[L^{q}(\Omega)\right]_{\text {sym }}^{2 \times 2}$ by $\underline{\underline{\mathcal{F}_{k}}} \in\left[L^{2}(\Omega)\right]_{\text {sym }}^{2 \times 2}$ such that $\left|\underline{\underline{\mathcal{F}}}-\underline{\underline{\mathcal{F}_{k}}}\right|_{0, q} \rightarrow 0$ as $k \rightarrow \infty$. As $\widehat{\widehat{V}}_{p} \subset \widehat{\underline{V}}_{2}$ it then follows that

$$
\left(\mathcal{Q} \underline{\underline{\mathcal{F}_{k}}}, \underline{\underline{\mathcal{H}}}\right)=\left(\mathcal{Q} \underline{\underline{\mathcal{F}_{k}}}, \mathcal{Q} \underline{\underline{\mathcal{H}}}\right)=\left(\underline{\underline{\mathcal{F}_{k}}}, \underline{Q} \underline{\underline{\mathcal{H}}}\right) \quad \forall \underline{\underline{\mathcal{H}}} \in\left[L^{p}(\Omega)\right]_{\mathrm{sym}}^{2 \times 2} .
$$

Hence we obtain that

$$
\left|\left(\mathcal{Q} \underline{\underline{\mathcal{F}_{k}}}, \underline{\underline{\mathcal{H}}}\right)\right| \leq\|\mathcal{Q}\|_{p}|\underline{\underline{\underline{\mathcal{H}}}}|_{0, p}\left|\underline{\underline{\mathcal{F}_{k}}}\right|_{0, q} \quad \Longrightarrow \quad\left|\mathcal{Q} \underline{\underline{\mathcal{F}_{k}}}\right|_{0, q} \leq\|\mathcal{Q}\|_{p}\left\|\underline{\underline{\mathcal{F}_{k}}}\right\|_{0, q} .
$$

Taking the weak limit of $\underline{f}_{\mathcal{F}_{k}}$ in $\widehat{\underline{V}}_{q}$, where $\underline{\underline{\mathcal{E}}}\left(\underline{f}_{\mathcal{F}_{k}}\right)=\mathcal{Q} \underline{\underline{\mathcal{F}_{k}}}$, we obtain that $(\mathcal{Q} \underline{\underline{\mathcal{F}}}, \underline{\underline{\mathcal{H}}})$ $=(\underline{\underline{\mathcal{F}}}, \mathcal{Q} \underline{\underline{\mathcal{H}}})$ for all $\underline{\underline{\mathcal{F}}} \in\left[L^{q}(\Omega)\right]_{\text {sym }}^{2 \times 2}$ and $\underline{\underline{\mathcal{H}}} \in\left[L^{p}(\Omega)\right]_{\text {sym }}^{2 \times 2}$. Hence $\mathcal{Q}$ defined on $\left[L^{q}(\bar{\Omega})\right]_{\mathrm{sym}}^{2 \times 2}$ is the dual operator to $\mathcal{Q}$ defined on $\left[L^{p}(\Omega)\right]_{\mathrm{sym}}^{2 \times 2}$ and therefore $\|\mathcal{Q}\|_{p}=$ $\|\mathcal{Q}\|_{q}$.

The Riesz-Thorin theorem, see [13], then implies that $\|\mathcal{Q}\|_{p} \leq\|\mathcal{Q}\|_{s}^{1-\alpha}\|\mathcal{Q}\|_{r}^{\alpha}$ for all $\frac{2+\delta}{1+\delta} \leq s \leq p \leq r \leq 2+\delta$ such that $\frac{1}{p}=(1-\alpha) \frac{1}{s}+\alpha \frac{1}{r}$ and $\alpha \in[0,1]$. It follows that $\log \|\mathcal{Q}\|_{p}$ is a convex function of $\frac{1}{p}$ and therefore $\|\mathcal{Q}\|_{p}$ is a continuous function of $p$ with $\|\mathcal{Q}\|_{2}=1$. Finally, it follows from (1.21) and (1.22) that $\underline{\underline{\mathcal{E}}}(\underline{z})=\mathcal{Q} \underline{\underline{\mathcal{G}_{z}}}$ 
and hence $|\underline{\underline{\mathcal{E}}}(\underline{z})|_{0, p} \leq\|\mathcal{Q}\|_{p}\left|\underline{\underline{\mathcal{G}_{z}}}\right|_{0, p}$. Therefore the desired result (1.20) follows from (1.21) with $\beta(p)=\|\mathcal{Q}\|_{p}$.

We note also for future reference the generalised Young's inequality

$(1.23) r s \leq \frac{1}{p}(\alpha r)^{p}+\frac{1}{q}\left(\alpha^{-1} s\right)^{q} \quad \forall r, s \in \mathbb{R}, \alpha \in \mathbb{R}_{>0}, p \in(1, \infty)$ with $\frac{1}{p}+\frac{1}{q}=1$.

Throughout $C$ denotes a generic constant independent of $h, \tau$ and $\varepsilon$, which denote the mesh and temporal discretization parameters and the regularization parameter, respectively. In addition $C\left(a_{1}, \ldots, a_{I}\right)$ denotes a constant depending on the arguments $\left\{a_{i}\right\}_{i=1}^{I}$. In the technical parts of this paper, we will frequently refer to a number of previously established results and definitions in order to derive an inequality. In each case, the results referred to are quoted in the exact order in which they need to be applied in the ensuing analysis. Finally, ${ }^{(\star)}$ denotes an expression with or without the superscript $\star$.

\section{Finite element approximation}

We consider the finite element approximation of $(\mathrm{P})$ under the following assumptions on the mesh:

(A) Let $\Omega$ be a convex polygonal domain. Let $\left\{\mathcal{T}^{h}\right\}_{h>0}$ be a quasi-uniform family of partitionings of $\Omega$ into disjoint open simplices $\sigma$ with $h_{\sigma}:=\operatorname{diam}(\sigma)$ and $h:=\max _{\sigma \in \mathcal{T}^{h}} h_{\sigma}$, so that $\bar{\Omega}=\bigcup_{\sigma \in \mathcal{T}^{h}} \bar{\sigma}$. In addition, it is assumed that all simplices $\sigma \in \mathcal{T}^{h}$ are right-angled.

Associated with $\mathcal{T}^{h}$ is the finite element space $S^{h}:=\left\{\chi \in C(\bar{\Omega}):\left.\chi\right|_{\sigma}\right.$ is linear $\left.\forall \sigma \in \mathcal{T}^{h}\right\} \subset H^{1}(\Omega)$. We introduce also $\underline{S}^{h}:=\left[S^{h}\right]^{2}, \underline{\widehat{S}}^{h}:=\underline{S}^{h} \cap \underline{\widehat{H}}^{1}(\Omega)$ and $K^{h}:=\left\{\chi \in S^{h}:|\chi| \leq 1\right.$ in $\left.\Omega\right\} \subset K:=\left\{\eta \in H^{1}(\Omega):|\eta| \leq 1\right.$ a.e. in $\left.\Omega\right\}$. Let $J$ be the set of nodes of $\mathcal{T}^{h}$ and $\left\{p_{j}\right\}_{j \in J}$ the coordinates of these nodes. Let $\left\{\chi_{j}\right\}_{j \in J}$ be the standard basis functions for $S^{h}$; that is $\chi_{j} \in K^{h}$ and $\chi_{j}\left(p_{i}\right)=\delta_{i j}$ for all $i, j \in J$. The right-angle constraint on the partitioning is required for our approximation of $b(\cdot)$, (see (2.2) below), but one consequence is that

$$
\int_{\sigma} \nabla \chi_{i} \cdot \nabla \chi_{j} \mathrm{~d} x \leq 0 \quad i \neq j, \quad \forall \sigma \in \mathcal{T}^{h} .
$$

We introduce $\pi^{h}: C(\bar{\Omega}) \rightarrow S^{h}$, the interpolation operator, such that $\left(\pi^{h} \eta\right)\left(p_{j}\right)=$ $\eta\left(p_{j}\right)$ for all $j \in J$. A discrete semi-inner product on $C(\bar{\Omega})$ is then defined by $\left(\eta_{1}, \eta_{2}\right)^{h}:=\int_{\Omega} \pi^{h}\left(\eta_{1}(x) \eta_{2}(x)\right) \mathrm{d} x=\sum_{j \in J} m_{j} \eta_{1}\left(p_{j}\right) \eta_{2}\left(p_{j}\right)$, where $m_{j}:=\left(1, \chi_{j}\right)>$ 0 . The induced discrete semi-norm is then $|\eta|_{h}:=\left[(\eta, \eta)^{h}\right]^{\frac{1}{2}}$, where $\eta \in C(\bar{\Omega})$. Both $(\cdot, \cdot)^{h}$ and $|\cdot|_{h}$ are naturally extended to vector-valued and matrix-valued functions. We introduce also the $L^{2}$ projection $Q^{h}: L^{2}(\Omega) \rightarrow S^{h}$ defined by $\left(Q^{h} \eta, \chi\right)^{h}=(\eta, \chi)$ for all $\chi \in S^{h}$.

We recall from [12, §2] the definition of the regularization $G_{\varepsilon} \in C^{2,1}(\mathbb{R})$ of $G$. Similarly to the approach in [47] and [35, we introduce $\Xi_{\varepsilon}: S^{h} \rightarrow\left[L^{\infty}(\Omega)\right]^{2 \times 2}$ approximating $b(\cdot) \mathcal{I}$, where $\mathcal{I} \in \mathbb{R}^{2 \times 2}$ is the identity matrix, such that for all $z^{h} \in S^{h}$ and a.e. in $\Omega$,

$$
\Xi_{\varepsilon}\left(z^{h}\right) \text { is symmetric and positive semi-definite, } \quad \Xi_{\varepsilon}\left(z^{h}\right) \nabla \pi^{h}\left[G_{\varepsilon}^{\prime}\left(z^{h}\right)\right]=\nabla z^{h} .
$$

The construction of $\Xi_{\varepsilon}$ can be found in [12, §2]. We note that it is this construction that requires the right-angle constraint on the partitioning $\mathcal{T}^{h}$. 
In addition to $\mathcal{T}^{h}$, let $0=t_{0}<t_{1}<\cdots<t_{N-1}<t_{N}=T$ be a partitioning of $[0, T]$ into possibly variable time steps $\tau_{n}:=t_{n}-t_{n-1}, n=1 \rightarrow N$. We set $\tau:=\max _{n=1 \rightarrow N} \tau_{n}$. For any given $\varepsilon \in(0,1)$, we then consider the following fully practical finite element approximation of $(\mathrm{P})$ :

$\left(\mathbf{P}_{\varepsilon}^{h, \tau}\right)$ For $n \geq 1$ find $\left\{\underline{U}_{\varepsilon}^{n}, \Theta_{\varepsilon}^{n}, W_{\varepsilon}^{n}\right\} \in \underline{\widehat{S}}^{h} \times K^{h} \times S^{h}$ such that

$$
\begin{array}{cc}
\left(c\left(\Theta_{\varepsilon}^{n-1}\right) \mathcal{C} \underline{\underline{\mathcal{E}}}\left(\underline{U_{\varepsilon}^{n}}\right), \underline{\underline{\mathcal{E}}}(\underline{\chi})\right)=\int_{\partial \Omega} \underline{g} \cdot \underline{\chi} \mathrm{d} s & \forall \underline{\chi} \in \underline{S}^{h}, \\
\gamma\left(\frac{\Theta_{\varepsilon}^{n}-\Theta_{\varepsilon}^{n-1}}{\tau_{n}}, \chi\right)^{h}+\left(\Xi_{\varepsilon}\left(\Theta_{\varepsilon}^{n-1}\right) \nabla W_{\varepsilon}^{n}, \nabla \chi\right)=0 & \forall \chi \in S^{h}, \\
\gamma\left(\nabla \Theta_{\varepsilon}^{n}, \nabla\left[\chi-\Theta_{\varepsilon}^{n}\right]\right) \geq\left(W_{\varepsilon}^{n}+\gamma^{-1} \Theta_{\varepsilon}^{n-1}, \chi-\Theta_{\varepsilon}^{n}\right)^{h} & \\
-\frac{1}{2}\left(c^{\prime}\left(\Theta_{\varepsilon}^{n-1}\right) \mathcal{C} \underline{\underline{\mathcal{E}}}\left(\underline{U}_{\varepsilon}^{n}\right): \underline{\underline{\mathcal{E}}}\left(\underline{U}_{\varepsilon}^{n}\right), \chi-\Theta_{\varepsilon}^{n}\right) & \forall \chi \in K^{h},
\end{array}
$$

where $\Theta_{\varepsilon}^{0} \in K^{h}$ is an approximation of $\theta^{0} \in K$, e.g., $\Theta_{\varepsilon}^{0} \equiv Q^{h} \theta^{0}$, or $\Theta_{\varepsilon}^{0} \equiv \pi^{h} \theta^{0}$ if $\theta^{0} \in C(\bar{\Omega})$.

Remark 2.1. We note that in the case $\mathcal{C} \equiv 0,(2.3 \mathrm{~b}, \mathrm{c})$ collapses to an approximation of the degenerate Cahn-Hilliard equation, $(1.12 \mathrm{a}-\mathrm{c})$ with $\mathcal{C} \equiv 0$. This is the same as the approximation in [12] in the absence of an electric field. Note that as $c^{\prime}$ is constant, the dependence on $\Theta_{\varepsilon}^{n-1}$ in $(2.3 \mathrm{C})$ is superfluous.

Below we recall some well-known results concerning $S^{h}$ for any $\sigma \in \mathcal{T}^{h}, \chi, z^{h} \in$ $S^{h}, m \in\{0,1\}, p \in[1, \infty], q \in[2, \infty)$ and $r \in(2, \infty]:$

$$
\begin{array}{rlrl}
|\chi|_{1, \sigma} & \leq C h_{\sigma}^{-1}|\chi|_{0, \sigma} ; & & \\
|\chi|_{m, s, \sigma} & \leq C h_{\sigma}^{-2\left(\frac{1}{p}-\frac{1}{s}\right)}|\chi|_{m, p, \sigma} & & \text { for any } s \in[p, \infty] ; \\
\left|\left(I-\pi^{h}\right) \eta\right|_{m, q, \sigma} & \leq C h^{1+\frac{2}{q}-m}|\eta|_{2, \sigma} & & \forall \eta \in H^{2}(\sigma) ; \\
\left|\left(I-\pi^{h}\right) \eta\right|_{m, r, \sigma} & \leq C h^{1-m}|\eta|_{1, r, \sigma} & \forall \eta \in W^{1, r}(\sigma) ; \\
\int_{\sigma} \chi^{2} \mathrm{~d} x & \leq \int_{\sigma} \pi^{h}\left[\chi^{2}\right] \mathrm{d} x \leq 4 \int_{\sigma} \chi^{2} \mathrm{~d} x ; & \\
\left|\left(\chi, z^{h}\right)-\left(\chi, z^{h}\right)^{h}\right| & \leq\left|\left(I-\pi^{h}\right)\left(\chi z^{h}\right)\right|_{0,1} \leq C h^{1+m}|\chi|_{m}\left|z^{h}\right|_{1} .
\end{array}
$$

It is convenient to introduce the "inverse Laplacian" operator $\mathcal{G}: Y \rightarrow Z$ such that

$$
(\nabla[\mathcal{G} z], \nabla \eta)=\langle z, \eta\rangle \quad \forall \eta \in H^{1}(\Omega),
$$

where $Y:=\left\{z \in\left(H^{1}(\Omega)\right)^{\prime}:\langle z, 1\rangle=0\right\}$ and $Z:=\left\{z \in H^{1}(\Omega):(z, 1)=0\right\}$. Here and throughout $\langle\cdot, \cdot\rangle$ denotes the duality pairing between $\left(H^{1}(\Omega)\right)^{\prime}$ and $H^{1}(\Omega)$, and its extension to the corresponding spaces of vector-valued functions. The wellposedness of $\mathcal{G}$ follows from the generalised Lax-Milgram theorem and a Poincaré inequality. As $\Omega$ is convex polygonal, we recall the well-known regularity result

$$
\|\mathcal{G} z\|_{2} \leq C|z|_{0} \quad \forall z \in L^{2}(\Omega) \cap Y .
$$

We define $Z^{h}:=\left\{z^{h} \in S^{h}:\left(z^{h}, 1\right)=0\right\} \subset Y^{h}:=\left\{z \in C(\bar{\Omega}):(z, 1)^{h}=0\right\} \subset Y$. Then, similarly to (2.10), we introduce $\mathcal{G}^{h}: Y^{h} \rightarrow Z^{h}$ such that

$$
\left(\nabla\left[\mathcal{G}^{h} z^{h}\right], \nabla \chi\right)=\left(z^{h}, \chi\right)^{h} \quad \forall \chi \in S^{h} .
$$


It is easily established from (2.10), (2.12), $\left\{\mathcal{T}^{h}\right\}_{h>0}$ being a regular partitioning, (2.11) and (2.9) that

$$
\left\|\left(\mathcal{G}-\mathcal{G}^{h}\right) z^{h}\right\|_{1} \leq C h\left|z^{h}\right|_{0} \quad \forall z^{h} \in S^{h} .
$$

We introduce the "discrete Laplacian" operator $\Delta^{h}: S^{h} \rightarrow Z^{h}$ such that

$$
\left(\Delta^{h} z^{h}, \chi\right)^{h}=-\left(\nabla z^{h}, \nabla \chi\right) \quad \forall \chi \in S^{h} .
$$

It follows from (2.8), (2.14), (2.5) and the quasi-uniformity assumption on $\mathcal{T}^{h}$ that

$$
\begin{aligned}
\left|\Delta^{h} z^{h}\right|_{0}^{2} & \leq\left|\Delta^{h} z^{h}\right|_{h}^{2}=-\left(\nabla z^{h}, \nabla\left(\Delta^{h} z^{h}\right)\right) \leq\left|z^{h}\right|_{1}\left|\Delta^{h} z^{h}\right|_{1} \\
& \leq C h^{-1}\left|z^{h}\right|_{1}\left|\Delta^{h} z^{h}\right|_{0} \leq C h^{-2}\left|z^{h}\right|_{1}^{2} \leq C h^{-4}\left|z^{h}\right|_{0}^{2} \quad \forall z^{h} \in S^{h} .
\end{aligned}
$$

Lemma 2.1. Let the assumptions $(A)$ hold. Then for all $z^{h} \in S^{h}$ we have that

$$
\begin{aligned}
& \left|z^{h}\right|_{1, s} \leq C\left|\Delta^{h} z^{h}\right|_{0}, \quad \text { for any } s \in(1, \infty), \\
& \left|z^{h}\right|_{1,4} \leq C\left|\Delta^{h} z^{h}\right|_{0}^{\frac{1}{2}}\left|z^{h}\right|_{1}^{\frac{1}{2}} .
\end{aligned}
$$

Furthermore

$$
\left|\Delta^{h}\left(\pi^{h} \eta\right)\right|_{0} \leq C|\eta|_{2} \quad \forall \eta \in H^{2}(\Omega) \text { with } \frac{\partial \eta}{\partial \nu}=0 \text { on } \partial \Omega .
$$

Proof. The proof of (2.16a) can be found in [10, Lemma 3.1], and the proof of (2.16b) is very similar. It follows from (2.14) and (2.12) that

$$
(I-f) z^{h}=-\mathcal{G}^{h}\left[\Delta^{h} z^{h}\right] \quad \forall z^{h} \in S^{h} .
$$

We have from (2.18), (1.17), (2.6), (2.5), (2.11) and (2.13) that

$$
\begin{aligned}
\left|z^{h}\right|_{1,4} & \leq\left|\mathcal{G}\left[\Delta^{h} z^{h}\right]\right|_{1,4}+\left|\left(I-\pi^{h}\right) \mathcal{G}\left[\Delta^{h} z^{h}\right]\right|_{1,4}+\left|\left(\pi^{h} \mathcal{G}-\mathcal{G}^{h}\right) \Delta^{h} z^{h}\right|_{1,4} \\
& \leq\left|\mathcal{G}\left[\Delta^{h} z^{h}\right]\right|_{1}^{\frac{1}{2}}\left\|\mathcal{G}\left[\Delta^{h} z^{h}\right]\right\|_{2}^{\frac{1}{2}}+C h^{\frac{1}{2}}\left|\mathcal{G}\left[\Delta^{h} z^{h}\right]\right|_{2}+C h^{-\frac{1}{2}}\left|\left(\pi^{h} \mathcal{G}-\mathcal{G}^{h}\right) \Delta^{h} z^{h}\right|_{1} \\
(2.19) & \leq\left|\mathcal{G}\left[\Delta^{h} z^{h}\right]\right|_{1}^{\frac{1}{2}}\left|\Delta^{h} z^{h}\right|_{0}^{\frac{1}{2}}+C h^{\frac{1}{2}}\left|\Delta^{h} z^{h}\right|_{0} \quad \forall z^{h} \in S^{h} .
\end{aligned}
$$

It follows from (2.18), (2.13) and (2.15) that for all $z^{h} \in S^{h}$

$$
\left|\mathcal{G}\left[\Delta^{h} z^{h}\right]\right|_{1} \leq\left|\mathcal{G}^{h}\left[\Delta^{h} z^{h}\right]\right|_{1}+\left|\left(\mathcal{G}-\mathcal{G}^{h}\right)\left[\Delta^{h} z^{h}\right]\right|_{1} \leq\left|z^{h}\right|_{1}+C h\left|\Delta^{h} z^{h}\right|_{0} \leq C\left|z^{h}\right|_{1} .
$$

Combining (2.19) and (2.15) yields that

$$
\left|z^{h}\right|_{1,4} \leq C\left|z^{h}\right|_{1}^{\frac{1}{2}}\left|\Delta^{h} z^{h}\right|_{0}^{\frac{1}{2}}+C h^{\frac{1}{2}}\left|\Delta^{h} z^{h}\right|_{0} \leq C\left|z^{h}\right|_{1}^{\frac{1}{2}}\left|\Delta^{h} z^{h}\right|_{0}^{\frac{1}{2}} \quad \forall z^{h} \in S^{h},
$$

and hence the desired result (2.16b).

Finally, it follows from (2.8), (2.14), (2.6), $\eta \in H^{2}(\Omega)$ with $\frac{\partial \eta}{\partial \nu}=0$ on $\partial \Omega$, and (2.4) that

$$
\begin{aligned}
\left|\Delta^{h}\left(\pi^{h} \eta\right)\right|_{0}^{2} & \leq\left|\Delta^{h}\left(\pi^{h} \eta\right)\right|_{h}^{2}=-\left(\nabla\left(\pi^{h} \eta\right), \nabla\left(\Delta^{h}\left(\pi^{h} \eta\right)\right)\right) \\
& =-\left(\nabla \eta, \nabla\left(\Delta^{h}\left(\pi^{h} \eta\right)\right)\right)+\left(\nabla\left(I-\pi^{h}\right) \eta, \nabla\left(\Delta^{h}\left(\pi^{h} \eta\right)\right)\right) \\
& \leq|\Delta \eta|_{0}\left|\Delta^{h}\left(\pi^{h} \eta\right)\right|_{0}+C h|\eta|_{2}\left|\nabla\left(\Delta^{h}\left(\pi^{h} \eta\right)\right)\right|_{0} \leq C|\eta|_{2}^{2},
\end{aligned}
$$

and hence the desired result (2.17).

Similarly to (2.14), we introduce $L^{h}: \underline{S}^{h} \rightarrow \underline{\widehat{S}}^{h}$ such that

$$
\left(L^{h} \underline{z}^{h}, \underline{\chi}\right)=-\left(\mathcal{C} \underline{\underline{\mathcal{E}}}\left(\underline{z}^{h}\right), \underline{\underline{\mathcal{E}}}(\underline{\chi})\right) \quad \forall \underline{\chi} \in \underline{S}^{h} .
$$


We introduce also $N_{\mathcal{C}}: \underline{X} \rightarrow \underline{\widehat{H}}^{1}(\Omega)$ and $N_{\mathcal{C}}^{h}: \underline{X} \rightarrow \underline{\widehat{S}}^{h}$, where $\underline{X}:=\left\{\underline{\eta} \in\left(\underline{H}^{1}(\Omega)\right)^{\prime}:\right.$ $\langle\underline{\eta}, \underline{v}\rangle=0 \quad \forall \underline{v} \in \underline{\mathrm{RM}}\}$, such that

$$
\begin{array}{ll}
\left(\mathcal{C} \underline{\underline{\mathcal{E}}}\left(N_{\mathcal{C}} \underline{\xi}\right), \underline{\underline{\mathcal{E}}}(\underline{\eta})\right)=\langle\underline{\xi}, \underline{\eta}\rangle & \forall \underline{\eta} \in \underline{H}^{1}(\Omega), \\
\left(\mathcal{C} \underline{\underline{\mathcal{E}}}\left(N_{\mathcal{C}}^{h} \underline{\xi}\right), \underline{\underline{\mathcal{E}}}(\underline{\chi})\right)=\langle\underline{\xi}, \underline{\chi}\rangle & \forall \underline{\chi} \in \underline{S}^{h} .
\end{array}
$$

As $\mathcal{C}$ satisfies (1.9) and (1.10), the well-posedness of these operators is easily demonstrated. As $\Omega$ is convex polygonal, we will assume the analogue of (2.11),

$$
\left\|N_{\mathcal{C}} \underline{\xi}\right\|_{2} \leq C|\underline{\xi}|_{0} \quad \forall \underline{\xi} \in \underline{L}^{2}(\Omega) \cap \underline{X} .
$$

If $\mathcal{C}$ is isotropic, (1.11), then the singularity exponents in $N_{\mathcal{C}} \xi$ do not depend on the Lamé moduli, and (2.23) follows immediately, for example, on combining [33. Theorem I] and [44, Lemma 3.2]. Unfortunately, if $\mathcal{C}$ is anisotropic, then the singularity exponents depend on the specific form of $\mathcal{C}$ and there is no general result of the type (2.23) in the literature. However, there is also no counterexample. For any particular material law, $\mathcal{C}$, and domain $\Omega$ the singularity exponents in $N_{\mathcal{C}} \xi$ can be computed, see, e.g., 20, and hence the assumption (2.23) can be tested.

We now have the analogues of (2.15), (2.16a) and (2.17).

Lemma 2.2. Let the assumptions $(A)$ hold and, if $\mathcal{C}$ is anisotropic, assume that (2.23) holds. Then for all $s \in(1, \infty)$ and for all $\underline{z}^{h} \in \underline{S}^{h}$ we have that

$$
\left|\underline{\underline{\mathcal{E}}}\left(\underline{z}^{h}\right)\right|_{0, s} \leq C\left|L^{h} \underline{z}^{h}\right|_{0} \leq C h^{-1}\left|\underline{z}^{h}\right|_{1} .
$$

Furthermore

$$
\left|L^{h}\left(\pi^{h} \underline{\eta}\right)\right|_{0} \leq C|\underline{\eta}|_{2} \quad \forall \underline{\eta} \in \underline{H}^{2}(\Omega) \quad \text { with } \quad \mathcal{C} \underline{\underline{\mathcal{E}}}(\underline{\eta}) \underline{\nu}=\underline{0} \text { on } \partial \Omega .
$$

Proof. It follows from (1.19), (1.10), (2.21), (2.22), (2.6) and (2.23) that

$$
\begin{gathered}
C\left\|\left(N_{\mathcal{C}}-N_{\mathcal{C}}^{h}\right) \underline{\xi}\right\|_{1}^{2} \leq C\left|\underline{\underline{\mathcal{E}}}\left(\left(N_{\mathcal{C}}-N_{\mathcal{C}}^{h}\right) \underline{\xi}\right)\right|_{0}^{2} \leq\left(\mathcal{C} \underline{\underline{\mathcal{E}}}\left(\left(N_{\mathcal{C}}-N_{C}^{h}\right) \underline{\xi}\right), \underline{\underline{\mathcal{E}}}\left(\left(N_{\mathcal{C}}-N_{\mathcal{C}}^{h}\right) \underline{\xi}\right)\right) \\
=\left(\mathcal{C} \underline{\underline{\mathcal{E}}}\left(\left(N_{\mathcal{C}}-N_{\mathcal{C}}^{h}\right) \underline{\xi}\right), \underline{\underline{\mathcal{E}}}\left(\left(I-\pi^{h}\right)\left(N_{\mathcal{C}} \underline{\xi}\right)\right)\right) \leq C\left|\underline{\underline{\mathcal{E}}}\left(\left(I-\pi^{h}\right)\left(N_{\mathcal{C}} \underline{\xi}\right)\right)\right|_{0}^{2} \\
\leq C\left|\left(I-\pi^{h}\right)\left(N_{\mathcal{C}} \underline{\xi}\right)\right|_{1}^{2} \leq C h^{2}\left\|N_{\mathcal{C}} \underline{\xi}\right\|_{2}^{2} \leq C h^{2}|\underline{\xi}|_{0}^{2} \quad \forall \underline{\xi} \in \underline{L}^{2}(\Omega) \cap \underline{X} .
\end{gathered}
$$

Let $\underline{z}^{h}=\left(\underline{z}^{h}-\underline{z}_{\mathrm{RM}}^{h}\right)+\underline{z}_{\mathrm{RM}}^{h}$ such that $\underline{z}_{\mathrm{RM}}^{h} \in \underline{\mathrm{RM}}$ and $\underline{z}^{h}-\underline{z}_{\mathrm{RM}}^{h} \in \underline{\widehat{S}}^{h}$. Then it follows from (2.22) and (1.19) that

$$
\underline{z}^{h}-\underline{z}_{\mathrm{RM}}^{h}=-N_{\mathcal{C}}^{h}\left(L^{h} \underline{z}^{h}\right) .
$$

Combining (2.26), (1.17), (2.7), (2.5) and the above-established bound yields for $s \in(2, \infty)$,

$$
\begin{array}{r}
\left|\underline{\mathcal{E}}\left(\underline{z}^{h}\right)\right|_{0, s}=\left|\underline{\underline{\mathcal{E}}}\left(N_{\mathcal{C}}^{h}\left(L^{h} \underline{z}^{h}\right)\right)\right|_{0, s} \leq\left|\underline{\underline{\mathcal{E}}}\left(N_{\mathcal{C}}\left(L^{h} \underline{z}^{h}\right)\right)\right|_{0, s}+\left|\underline{\underline{\mathcal{E}}}\left(\left(I-\pi^{h}\right) N_{\mathcal{C}}\left(L^{h} \underline{z}^{h}\right)\right)\right|_{0, s} \\
+\left|\underline{\underline{\mathcal{E}}}\left(\pi^{h}\left[N_{\mathcal{C}}\left(L^{h} \underline{z}^{h}\right)\right]-N_{\mathcal{C}}^{h}\left(L^{h} \underline{z}^{h}\right)\right)\right|_{0, s} \\
\leq C\left\|N_{\mathcal{C}}\left(L^{h} \underline{z}^{h}\right)\right\|_{2}+C h^{-\left(1-\frac{2}{s}\right)}\left|\pi^{h}\left[N_{\mathcal{C}}\left(L^{h} \underline{z}^{h}\right)\right]-N_{\mathcal{C}}^{h}\left(L^{h} \underline{z}^{h}\right)\right|_{1} \leq C\left|L^{h} \underline{z}^{h}\right|_{0}
\end{array}
$$

and hence the first inequality in (2.24).

It follows from (2.20) and (2.4) that

$$
\begin{aligned}
\left|L^{h} \underline{z}^{h}\right|_{0}^{2} & =-\left(\mathcal{C} \underline{\underline{\mathcal{E}}}\left(\underline{z}^{h}\right), \underline{\underline{\mathcal{E}}}\left(L^{h} \underline{z}^{h}\right)\right) \leq C\left|\underline{\underline{\mathcal{E}}}\left(\underline{z}^{h}\right)\right|_{0}\left|\underline{\underline{\mathcal{E}}}\left(L^{h} \underline{z}^{h}\right)\right|_{0} \\
& \leq C h^{-1}\left|\underline{\underline{\mathcal{E}}}\left(\underline{z}^{h}\right)\right|_{0}\left|L^{h} \underline{z}^{h}\right|_{0} \leq C h^{-2}\left|\underline{\underline{\mathcal{E}}}\left(\underline{\underline{z}}^{h}\right)\right|_{0}^{2} \leq C h^{-2}\left|\underline{z}^{h}\right|_{1}^{2}
\end{aligned}
$$

and hence the second inequality in (2.24). 
Finally, it follows from (2.8), (2.20), (2.6), $\underline{\eta} \in \underline{H}^{2}(\Omega)$ with $\mathcal{C} \underline{\underline{\mathcal{E}}}(\underline{\eta}) \underline{\nu}=\underline{0}$ on $\partial \Omega$, and (2.4) that

$$
\begin{aligned}
\left|L^{h}\left(\pi^{h} \underline{\eta}\right)\right|_{0}^{2} & \leq\left|L^{h}\left(\pi^{h} \underline{\eta}\right)\right|_{h}^{2}=-\left(\mathcal{C} \underline{\underline{\mathcal{E}}}\left(\pi^{h} \underline{\eta}\right), \underline{\underline{\mathcal{E}}}\left(L^{h}\left(\pi^{h} \underline{\eta}\right)\right)\right) \\
& =-\left(\mathcal{C} \underline{\underline{\mathcal{E}}}(\underline{\eta}), \underline{\underline{\mathcal{E}}}\left(L^{h}\left(\pi^{h} \underline{\eta}\right)\right)\right)+\left(\mathcal{C} \underline{\underline{\mathcal{E}}}\left(\left(I-\pi^{h}\right) \underline{\eta}\right), \underline{\underline{\mathcal{E}}}\left(L^{h}\left(\pi^{h} \underline{\eta}\right)\right)\right) \\
& \leq C|\underline{\eta}|_{2}\left|L^{h}\left(\pi^{h} \underline{\eta}\right)\right|_{0}+C h|\underline{\eta}|_{2}\left|\nabla\left(L^{h}\left(\pi^{h} \underline{\eta}\right)\right)\right|_{0} \leq C|\underline{\eta}|_{2}^{2} .
\end{aligned}
$$

Hence we have the desired result (2.25).

We introduce the projection operator $P^{h}: \underline{W}^{1,1}(\Omega) \rightarrow \underline{\widehat{S}}^{h}$ such that

$$
\left(\underline{\underline{\mathcal{E}}}\left(\underline{z}-P^{h} \underline{z}\right), \underline{\underline{\mathcal{E}}} \underline{\chi} \underline{)}\right)=0 \quad \forall \underline{\chi} \in \underline{S}^{h} .
$$

It is crucial for our analysis to prove the following result.

Lemma 2.3. Let the assumptions ( $A$ ) hold and let $\delta \in \mathbb{R}_{>0}$ be as defined in Lemma 1.1. Then there exists $h_{0} \in \mathbb{R}_{>0}$ and $a \widehat{\beta} \in C\left(\left[\frac{2+\delta}{1+\delta}, \infty\right)\right)$ such that for all $p \in$ $\left[\frac{2+\delta}{1+\delta}, \infty\right)$ and for all $h \in\left(0, h_{0}\right)$,

$$
\left|\underline{\underline{\mathcal{E}}}\left(P^{h} \underline{z}\right)\right|_{0, p} \leq \widehat{\beta}(p)|\underline{\underline{\mathcal{E}}}(\underline{z})|_{0, p} \quad \forall \underline{z} \in \underline{\widehat{V}}_{p}
$$

with $\widehat{\beta}(p) \geq 1$ and $\widehat{\beta}(p) \rightarrow \widehat{\beta}(2)=1$ as $p \rightarrow 2$.

Proof. We adapt the proof for the Laplacian with homogeneous Dirichlet boundary conditions given in [17, Chapter 8], which is based on the approach in [42]. As the proof is long, we break it up into three parts, similarly to [17, Chapter 8].

1. Reduction of (2.28) to the weighted error estimate (2.37). Given $\mathcal{T}^{h}$ and any $y \in \Omega$, let $\sigma_{y} \in \mathcal{T}^{h}$ be such that $y \in \sigma_{y}$. We then introduce $\delta_{y}^{h} \in C_{0}^{\infty}(\bar{\Omega})$ with $\operatorname{supp}\left(\delta_{y}^{h}\right) \subset \sigma_{y}$ such that

$$
\int_{\sigma_{y}} \delta_{y}^{h} \mathrm{~d} x=1 \quad \text { and } \quad\left\|\delta_{y}^{h}\right\|_{m, \infty, \sigma_{y}} \leq C h^{-(2+m)} \quad \forall m \in \mathbb{N} .
$$

For $i, j \in\{1,2\}$, let $\underline{f}_{y, i j} \in \underline{\underline{H}}^{1}(\Omega)$ be such that

$$
\left(\underline{\underline{\mathcal{E}}}\left(\underline{f}_{y, i j}\right), \underline{\underline{\mathcal{E}}}(\underline{\eta})\right)=\left(\delta_{y}^{h},[\underline{\underline{\mathcal{E}}}(\underline{\eta})]_{i j}\right) \quad \forall \underline{\eta} \in \underline{H}^{1}(\Omega) .
$$

It follows from (1.14) and (1.19) that (2.30) is well-posed. We have from (2.29), (2.27) and (2.30) for all $y \in \Omega$ and for $i, j \in\{1,2\}$ that

$$
\begin{aligned}
{\left[\underline{\underline{\mathcal{E}}}\left(P^{h} \underline{z}\right)\right]_{i j}(y) } & =\left(\delta_{y}^{h},\left[\underline{\underline{\mathcal{E}}}\left(P^{h} \underline{z}\right)\right]_{i j}\right)=\left(\underline{\underline{\mathcal{E}}}\left(\underline{f}_{y, i j}\right), \underline{\underline{\mathcal{E}}}\left(P^{h} \underline{z}\right)\right)=\left(\underline{\underline{\mathcal{E}}}\left(P^{h} \underline{f}_{y, i j}\right), \underline{\underline{\mathcal{E}}}(\underline{z})\right) \\
(2.31) \quad & \left(\delta_{y}^{h},[\underline{\underline{\mathcal{E}}}(\underline{z})]_{i j}\right)+\left(\underline{\underline{\mathcal{E}}}\left(\left[P^{h}-I\right] \underline{f}_{y, i j}\right), \underline{\underline{\mathcal{E}}}(\underline{z})\right) \quad \forall \underline{z} \in \underline{H}^{1}(\Omega) .
\end{aligned}
$$

For any $y \in \Omega$ and any constant $\rho \geq 1$, we introduce the weight function

$$
\omega_{y, \rho}(x):=\left(|x-y|^{2}+\rho^{2} h^{2}\right)^{\frac{1}{2}} .
$$

It is easily verified for any $\alpha \in \mathbb{R}$ that

$$
\max _{\sigma \in \mathcal{T}^{h}}\left(\sup _{x \in \sigma}\left[\omega_{y, \rho}(x)\right]^{\alpha} / \inf _{x \in \sigma}\left[\omega_{y, \rho}(x)\right]^{\alpha}\right) \leq C, \quad\left|\omega_{y, \rho}^{\alpha}\right|_{0, \infty} \leq C \max \left\{1,(\rho h)^{\alpha}\right\}
$$

and

$$
\left|\frac{\partial^{m}}{\partial x_{i}^{m}}\left[\omega_{y, \rho}(x)\right]^{\alpha}\right| \leq C(\alpha)\left[\omega_{y, \rho}(x)\right]^{\alpha-m} \quad \forall x \in \Omega, \forall m \in \mathbb{N}, i \in\{1,2\},
$$


where the positive constant $C(\alpha)$ depends continuously on $\alpha$ and is independent of the choice of $y \in \Omega$ and $\rho \geq 1$. It follows immediately from (2.6) and (2.33a) that for all $\sigma \in \mathcal{T}^{h}, \alpha \in \mathbb{R}, m \in\{0,1\}, i \in\{1,2\}$ and $\eta \in H^{2}(\sigma)$,

$$
\int_{\sigma} \omega_{y, \rho}^{\alpha}\left[\frac{\partial^{m}}{\partial x_{i}^{m}}\left[\left(I-\pi^{h}\right) \eta\right]\right]^{2} \mathrm{~d} x \leq C h^{2(2-m)} \int_{\sigma} \omega_{y, \rho}^{\alpha}\left[\left(\frac{\partial^{2} \eta}{\partial x_{1}^{2}}\right)^{2}+\left(\frac{\partial^{2} \eta}{\partial x_{1} \partial x_{2}}\right)^{2}+\left(\frac{\partial^{2} \eta}{\partial x_{2}^{2}}\right)^{2}\right] \mathrm{d} x .
$$

It follows from (2.31), a Hölder inequality and (2.33a) that for any $p \in(2, \infty)$, $\alpha>0$ and $\rho \geq 1$,

$$
\begin{aligned}
\left|\underline{\underline{\mathcal{E}}}\left(P^{h} \underline{z}\right)\right|_{0, p} & \leq C\left[1+\left(\sup _{y \in \Omega} \int_{\Omega} \omega_{y, \rho}^{-(\alpha+2)} \mathrm{d} x\right)^{\frac{1}{2}} M_{\rho, \alpha}^{h}\right]|\underline{\underline{\mathcal{E}}}(\underline{z})|_{0, p} \\
& \leq C\left[1+\alpha^{-\frac{1}{2}}(\rho h)^{-\frac{\alpha}{2}} M_{\rho, \alpha}^{h}\right]|\underline{\underline{\mathcal{E}}}(\underline{z})|_{0, p} \quad \forall \underline{z} \in \underline{\widehat{V}}_{p},
\end{aligned}
$$

where

$$
M_{\rho, \alpha}^{h}:=\max _{i, j=1,2} \sup _{y \in \Omega}\left\{\int_{\Omega} \omega_{y, \rho}^{\alpha+2}\left|\underline{\underline{\mathcal{E}}}\left(\left[I-P^{h}\right] \underline{f} y, i j\right)\right|^{2} \mathrm{~d} x\right\}^{\frac{1}{2}} .
$$

The goal is to prove the analogue of [17, Lemma 8.2.6]; that is, for appropriate $\alpha>0$ and $\rho$ sufficiently large that there exists an $h_{0}$ such that

$$
M_{\rho, \alpha}^{h} \leq C h^{\frac{\alpha}{2}} \quad \forall h \in\left(0, h_{0}\right) .
$$

It would then follow from (2.35) and (2.37) that (2.28) holds with $\widehat{\beta}(p)=C_{1}$ for all $p \in(2, \infty)$, for some constant $C_{1}$. In addition, it would follow from (1.20), (2.27) and the above bound for $p \in(2, \infty)$ that for $p \in\left[\frac{2+\delta}{1+\delta}, 2\right)$ and for all $\underline{z} \in \underline{\underline{V}}_{p}$,

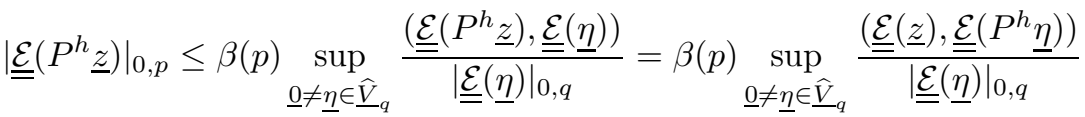

$$
\begin{aligned}
& \leq \beta(p) C_{1}|\underline{\underline{\mathcal{E}}}(\underline{z})|_{0, p},
\end{aligned}
$$

where $\frac{1}{p}+\frac{1}{q}=1$. As (2.28) trivially holds with $\widehat{\beta}(2)=1$ from inspecting (2.27), it follows that (2.28) holds with $\widehat{\beta}(p)=C_{2}$ for all $p \in\left[\frac{2+\delta}{1+\delta}, \infty\right)$, for some constant $C_{2}$. Moreover, the desired result (2.28) holds for all $p \in\left[\frac{2+\delta}{1+\delta}, \infty\right)$ by applying the Riesz-Thorin theorem as in Lemma 1.1 to the $P^{h}$ induced mapping that takes $\underline{\underline{\mathcal{E}}}(\underline{z}) \in\left[L^{p}(\Omega)\right]_{\mathrm{sym}}^{2 \times 2}$ to $\underline{\mathcal{E}}\left(P^{h} \underline{z}\right) \in\left[L^{p}(\Omega)\right]_{\mathrm{sym}}^{2 \times 2}$.

2. Reduction of (2.37) to the weighted regularity bound (2.50). Let $\underline{\mathcal{Y}}:=\left\{\{\underline{\xi}, \underline{\zeta}\} \in\left(\underline{H}^{1}(\Omega)\right)^{\prime} \times \underline{L}^{2}(\partial \Omega):\langle\underline{\xi}, \underline{v}\rangle+\int_{\partial \Omega} \underline{\zeta} \cdot \underline{v} \mathrm{~d} s=0 \quad \forall \underline{v} \in \underline{\mathrm{RM}}\right\}$. Then $N: \underline{\mathcal{Y}} \rightarrow \underline{\hat{H}}^{1}(\Omega)$ is such that

$$
(\underline{\underline{\mathcal{E}}}(N(\underline{\xi}, \underline{\zeta})), \underline{\underline{\mathcal{E}}}(\underline{\eta}))=\langle\underline{\xi}, \underline{\eta}\rangle+\int_{\partial \Omega} \underline{\zeta} \cdot \underline{\eta} \mathrm{d} s \quad \forall \underline{\eta} \in \underline{H}^{1}(\Omega) .
$$

Let $\partial \Omega \equiv \bigcup_{j=1}^{J_{B}} \overline{\partial_{j} \Omega}$ and $\partial_{j} \Omega \cap \partial_{k} \Omega=\emptyset$ for $j \neq k$; with $\underline{\nu}^{(j)}$ the outward unit normal to $\partial_{j} \Omega$. In addition, let the largest inner angle $\omega$ of the convex polygonal domain $\Omega$ be such that $\omega \leq \frac{r}{2 r-1} \pi$ for some $r>1$. Then, similarly to (2.23), on combining [33, Theorem I], $\varphi(z):=\sin ^{2}(\omega z)-z^{2} \sin \omega \Rightarrow \varphi(i z)=\widetilde{\varphi}(z):=z^{2} \sin ^{2} \omega-\sinh ^{2}(\omega z)$, and the fact that $\widetilde{\varphi}(z)$ has no roots such that $|\operatorname{Im}(z)| \leq \frac{\pi}{\omega}$, apart from the double 
root at $z=0$ and the simple roots at $z= \pm i$ (see [44, Lemma 3.2]) we have for $p \in(1,2 r]$ that for all $\{\underline{\xi}, \underline{\zeta}\} \in\left(\underline{L}^{p}(\Omega) \times \prod_{j=1}^{J_{B}} \underline{W}^{1-\frac{1}{p}, p}\left(\partial_{j} \Omega\right)\right) \cap \underline{\mathcal{Y}}$,

$$
\|N(\underline{\xi}, \underline{\zeta})\|_{2, p} \leq C\left[|\underline{\xi}|_{0, p}+\sum_{j=1}^{J_{B}}\|\underline{\zeta}\|_{1-\frac{1}{p}, p, \partial_{j} \Omega}\right],
$$

provided that the compatibility condition, [33, (1.5)],

$$
\left.\underline{\zeta}\right|_{\partial_{j} \Omega} \cdot \underline{\nu}^{(j+1)}=\left.\underline{\zeta}\right|_{\partial_{j+1} \Omega} \cdot \underline{\nu}^{(j)} \text { at every vertex } S_{j} \text { of } \Omega
$$

holds (in the integral sense if $p=2$ ).

For fixed $y \in \Omega$ and $i, j \in\{1,2\}$, let $\underline{e}:=\left(I-P^{h}\right) \underline{f}_{y, i j} \in \underline{\widehat{H}}^{1}(\Omega), \underline{e}^{A}:=$ $\left(I-\pi^{h}\right) \underline{f}_{y, i j} \in \underline{H}^{1}(\Omega)$ and $\underline{e}^{h}:=\left(\pi^{h}-P^{h}\right) \underline{f}_{y, i j} \in \underline{S}^{h}$. We note that

$$
\underline{\underline{\mathcal{E}}}(\eta \underline{z})=\eta \underline{\underline{\mathcal{E}}}(\underline{z})+\frac{1}{2}[\underline{z} \otimes(\nabla \eta)+(\nabla \eta) \otimes \underline{z}],
$$

where $\underline{a} \otimes \underline{b}:=\underline{a} \underline{b}^{T}$ for all $\underline{a}, \underline{b} \in \mathbb{R}^{2}$. It then follows from (2.42), (2.27), (1.23), (2.33a,b), (2.34) and (2.4) for any $y \in \Omega, i, j \in\{1,2\}, \alpha>0$ and $\rho \geq 1$ that

$$
\begin{aligned}
&\left(\omega_{y, \rho}^{\alpha+2} \underline{\underline{\mathcal{E}}}(\underline{e}), \underline{\underline{\mathcal{E}}}(\underline{e})\right)=\left(\underline{\underline{\mathcal{E}}}(\underline{e}), \underline{\underline{\mathcal{E}}}\left(\omega_{y, \rho}^{\alpha+2} \underline{e}^{A}\right)\right.\left.+\underline{\underline{\mathcal{E}}}\left(\left(I-\pi^{h}\right)\left[\omega_{y, \rho}^{\alpha+2} \underline{e}^{h}\right]\right)\right) \\
&-\frac{1}{2}\left(\underline{\underline{\mathcal{E}}}(\underline{e}),\left[\underline{e} \otimes\left(\nabla \omega_{y, \rho}^{\alpha+2}\right)+\left(\nabla \omega_{y, \rho}^{\alpha+2}\right) \otimes \underline{e}\right]\right) \\
& \leq C(\alpha)\left[\int_{\Omega}\left[\omega_{y, \rho}^{\alpha+2}\left|\underline{\underline{\mathcal{E}}}\left(\underline{e}^{A}\right)\right|^{2}+\omega_{y, \rho}^{\alpha}\left|\underline{e}^{A}\right|^{2}\right] \mathrm{d} x+\int_{\Omega} \omega_{y, \rho}^{\alpha}\left|\underline{e}^{h}\right|^{2} \mathrm{~d} x\right. \\
& \\
&\left.\quad+\int_{\Omega} \omega_{y, \rho}^{-(\alpha+2)}\left|\underline{\underline{\mathcal{E}}}\left(\left(I-\pi^{h}\right)\left[\omega_{y, \rho}^{\alpha+2} \underline{e}^{h}\right]\right)\right|^{2} \mathrm{~d} x\right] \\
&(2.43) \quad C(\alpha)\left[\int_{\Omega}\left[\omega_{y, \rho}^{\alpha+2}\left|\underline{\underline{\mathcal{E}}}\left(\underline{e}^{A}\right)\right|^{2}+\omega_{y, \rho}^{\alpha}\left|\underline{e}^{A}\right|^{2}\right] \mathrm{d} x+\int_{\Omega} \omega_{y, \rho}^{\alpha}\left|\underline{e}^{h}\right|^{2} \mathrm{~d} x\right] .
\end{aligned}
$$

Let $\underline{\psi}=N\left(\left(I-P_{\mathrm{RM}}\right)\left(\omega_{y, \rho}^{\alpha} \underline{e}\right), \underline{0}\right)$, where $P_{\mathrm{RM}}: \underline{L}^{2}(\Omega) \rightarrow \underline{\mathrm{RM}}$ is such that

$$
\left(\left(I-P_{\mathrm{RM}}\right) \underline{z}, \underline{\eta}\right)=0 \quad \forall \underline{\eta} \in \underline{\mathrm{RM}} .
$$

It follows from (1.19), (1.14) and (2.44) that

$$
\begin{aligned}
|\underline{v}|_{1} & \leq\left|\left(I-P_{\mathrm{RM}}\right) \underline{v}\right|_{1}+\left|P_{\mathrm{RM}} \underline{v}\right|_{1} \leq C\left|\underline{\underline{\mathcal{E}}}\left(\left(I-P_{\mathrm{RM}}\right) \underline{v}\right)\right|_{0}+\left|P_{\mathrm{RM}} \underline{v}\right|_{1} \\
& \leq C\left[|\underline{\underline{\mathcal{E}}}(\underline{v})|_{0}+\left|P_{\mathrm{RM}} \underline{v}\right|_{0}\right] \leq C\left[|\underline{\underline{\mathcal{E}}}(\underline{v})|_{0}+|\underline{v}|_{0}\right] \quad \forall \underline{v} \in \underline{H}^{1}(\Omega) .
\end{aligned}
$$

We have, on noting (2.39) and (2.27), that for all $\varsigma>0$,

$$
\begin{aligned}
\left(\omega_{y, \rho}^{\alpha} \underline{e}, \underline{e}\right) & =(\underline{\underline{\mathcal{E}}}(\underline{\psi}), \underline{\underline{\mathcal{E}}}(\underline{e}))=\left(\underline{\underline{\mathcal{E}}}\left(\left(I-\pi^{h}\right) \underline{\psi}\right), \underline{\underline{\mathcal{E}}}(\underline{e})\right) \\
& \leq \varsigma\left(\omega_{y, \rho}^{\alpha+2} \underline{\underline{\mathcal{E}}}(\underline{e}), \underline{\underline{\mathcal{E}}}(\underline{e})\right)+C \varsigma^{-1} \int_{\Omega} \omega_{y, \rho}^{-(\alpha+2)}\left|\underline{\underline{\mathcal{E}}}\left(\left(I-\pi^{h}\right) \underline{\psi}\right)\right|^{2} \mathrm{~d} x .
\end{aligned}
$$

It follows from (2.34) and (2.32) that

$$
\begin{gathered}
\int_{\Omega} \omega_{y, \rho}^{-(\alpha+2)}\left|\underline{\underline{\mathcal{E}}}\left(\left(I-\pi^{h}\right) \underline{\psi}\right)\right|^{2} \mathrm{~d} x \leq C h^{2} \sum_{k, \ell=1}^{2} \int_{\Omega} \omega_{y, \rho}^{-(\alpha+2)}\left|\frac{\partial^{2} \underline{\psi}}{\partial x_{k}} \underline{\partial x_{\ell}}\right|^{2} \mathrm{~d} x \\
\leq C h^{2}\left(\int_{\Omega} \omega_{y, \rho}^{-(\alpha+2) r^{\prime}} \mathrm{d} x\right)^{\frac{1}{r^{\prime}}}\|\underline{\psi}\|_{2,2 r}^{2} \leq C(\alpha) \rho^{-2}(\rho h)^{\frac{2}{r^{\prime}}-\alpha}\|\underline{\psi}\|_{2,2 r}^{2}
\end{gathered}
$$


where $r$ is as defined in (2.40) and $\frac{1}{r}+\frac{1}{r^{\prime}}=1$. Next we note that (2.40), (1.17), (1.19), (2.32), (2.42) and (2.33b) yield, on assuming that $\alpha \in\left(0, \frac{2(r-1)}{r}\right)$,

$$
\begin{aligned}
\|\underline{\psi}\|_{2,2 r}^{2} & \leq C\left|\left(I-P_{\mathrm{RM}}\right)\left(\omega_{y, \rho}^{\alpha} \underline{e}\right)\right|_{0,2 r}^{2} \leq C\left\|\left(I-P_{\mathrm{RM}}\right)\left(\omega_{y, \rho}^{\alpha} \underline{e}\right)\right\|_{1, \frac{2 r}{r+1}}^{2} \\
& \leq C\left|\underline{\underline{\mathcal{E}}}\left(\omega_{y, \rho}^{\alpha} \underline{e}\right)\right|_{0, \frac{2 r}{r+1}}^{2} \leq C\left(\int_{\Omega} \omega_{y, \rho}^{(\alpha-2) r} \mathrm{~d} x\right)^{\frac{1}{r}} \int_{\Omega} \omega_{y, \rho}^{2-\alpha}\left|\underline{\underline{\mathcal{E}}}\left(\omega_{y, \rho}^{\alpha} \underline{e}\right)\right|^{2} \mathrm{~d} x \\
& \leq C(\alpha)(\rho h)^{\alpha-2+\frac{2}{r}}\left[\left(\omega_{y, \rho}^{\alpha+2} \underline{\underline{\mathcal{E}}}(\underline{e}), \underline{\underline{\mathcal{E}}}(\underline{e})\right)+\left(\omega_{y, \rho}^{\alpha} \underline{e}, \underline{e}\right)\right] .
\end{aligned}
$$

Therefore for any fixed $\alpha \in\left(0, \frac{2(r-1)}{r}\right)$, we have for all $y \in \Omega, i, j \in\{1,2\}, \rho>\rho_{0}(\alpha)$ and $h>0$ on combining (2.43), (2.46) with $\varsigma$ sufficiently small, (2.47) and (2.48) that

$$
\left(\omega_{y, \rho}^{\alpha+2} \underline{\underline{\mathcal{E}}}(\underline{e}), \underline{\underline{\mathcal{E}}}(\underline{e})\right) \leq C(\alpha, \rho) \int_{\Omega}\left[\omega_{y, \rho}^{\alpha+2}\left|\underline{\underline{\mathcal{E}}}\left(\underline{e}^{A}\right)\right|^{2}+\omega_{y, \rho}^{\alpha}\left|\underline{e}^{A}\right|^{2}\right] \mathrm{d} x .
$$

Hence the desired result (2.37) follows from (2.36), (2.49) and (2.34) if we can show for any $y \in \Omega, i, j \in\{1,2\}, \alpha \in(0,1), \rho \geq 1$ and $h>0$ that

$$
\max _{k, \ell=1,2} \int_{\Omega} \omega_{y, \rho}^{\alpha+2}\left|\frac{\partial^{2} \underline{y}, i j}{\partial x_{k} \partial x_{\ell}}\right|^{2} \mathrm{~d} x \leq C(\alpha, \rho) h^{\alpha-2} .
$$

3. Proof of (2.50). First, we have from (2.33b) that

$$
\begin{aligned}
& \max _{k, \ell=1,2} \int_{\Omega} \omega_{y, \rho}^{\alpha+2}\left|\frac{\partial^{2} \underline{\underline{f}}}{\partial x_{k} \partial x_{\ell}}\right|^{2} \mathrm{~d} x \\
& \quad \leq C(\alpha)\left[\left|\omega_{y, \rho}^{\frac{\alpha}{2}+1} \underline{f}_{y, i j}\right|_{2}^{2}+\int_{\Omega}\left[\omega_{y, \rho}^{\alpha}\left|\nabla \underline{f}_{y, i j}\right|^{2}+\omega_{y, \rho}^{\alpha-2}\left|\underline{f}_{y, i j}\right|^{2}\right] \mathrm{d} x\right] .
\end{aligned}
$$

Second, it follows from (2.44), (1.14), (2.42), the symmetry of $\underline{\underline{\mathcal{E}}}(\cdot)$ and (2.30) that $\left(I-P_{\mathrm{RM}}\right)\left(\omega_{y, \rho}^{\frac{\alpha}{2}+1} \underline{f}_{y, i j}\right) \in \underline{\widehat{H}}^{1}(\Omega)$ solves for all $\underline{\eta} \in \underline{H}^{1}(\Omega)$,

$$
\begin{aligned}
(\underline{\underline{\mathcal{E}}} & \left.\left.\left(I-P_{\mathrm{RM}}\right)\left(\omega_{y, \rho}^{\frac{\alpha}{2}+1} \underline{f}_{y, i j}\right)\right), \underline{\underline{\mathcal{E}}}(\underline{\eta})\right)=\left(\underline{\underline{\mathcal{E}}}\left(\omega_{y, \rho}^{\frac{\alpha}{2}+1} \underline{f}_{y, i j}\right), \underline{\underline{\mathcal{E}}}(\underline{\eta})\right) \\
= & \left(\underline{\underline{\mathcal{E}}}\left(\underline{f}_{y, i j}\right), \underline{\underline{\mathcal{E}}}\left(\omega_{y, \rho}^{\frac{\alpha}{2}+1} \underline{\eta}\right)\right)+\frac{1}{2}\left(\left[\underline{f}_{y, i j} \otimes \nabla\left(\omega_{y, \rho}^{\frac{\alpha}{2}+1}\right)+\nabla\left(\omega_{y, \rho}^{\frac{\alpha}{2}+1}\right) \otimes \underline{f}_{y, i j}\right], \underline{\underline{\mathcal{E}}}(\underline{\eta})\right) \\
= & -\frac{1}{2}\left(\left[\underline{e}_{i, i j} \otimes \underline{e}_{j}+\underline{e}_{j} \otimes \underline{e}_{i}^{\frac{\alpha}{2}+1}\right] \nabla \delta_{y}^{h}, \omega_{y, \rho}^{\frac{\alpha}{2}+1} \underline{\eta}\right) \\
& -\left(\underline{\underline{\mathcal{E}}}_{y}\left(\underline{f}_{y, i j}\right) \nabla\left(\omega_{y, \rho}^{\frac{\alpha}{2}+1}\right)+\frac{1}{2} \nabla \cdot\left[\underline{f}_{y, i j} \otimes \nabla\left(\omega_{y, \rho}^{\frac{\alpha}{2}+1}\right)+\nabla\left(\omega_{y, \rho}^{\frac{\alpha}{2}+1}\right) \otimes \underline{f}_{y, i j}\right], \underline{\eta}\right) \\
& +\frac{1}{2} \int_{\partial \Omega}\left[\left[\underline{f}_{y, i j} \otimes \nabla\left(\omega_{y, \rho}^{\frac{\alpha}{2}+1}\right)+\nabla\left(\omega_{y, \rho}^{\frac{\alpha}{2}+1}\right) \otimes \underline{f}_{y, i j}\right] \underline{\nu}\right] \cdot \underline{\eta} \mathrm{d} s .
\end{aligned}
$$

Noting (2.39) and (2.41), and applying the bounds (2.40), (2.33b) and the trace inequality $\|\cdot\|_{\frac{1}{2}, \partial_{k} \Omega} \leq C\|\cdot\|_{1, \Omega}$ to (2.52) yields that

$$
\begin{gathered}
\left|\omega_{y, \rho}^{\frac{\alpha}{2}+1} \underline{f}_{y, i j}\right|_{2} \leq C\left[\left|\omega_{y, \rho}^{\frac{\alpha}{2}+1} \nabla \delta_{y}^{h}\right|_{0}+\left|\omega_{y, \rho}^{\frac{\alpha}{2}} \nabla \underline{f}_{y, i j}\right|_{0}+\left|\omega_{y, \rho}^{\frac{\alpha}{2}-1} \underline{f}_{y, i j}\right|_{0}\right. \\
\left.+\sum_{k=1}^{J_{B}}\left\|\left[\underline{f}_{y, i j} \otimes \nabla\left(\omega_{y, \rho}^{\frac{\alpha}{2}+1}\right)+\nabla\left(\omega_{y, \rho}^{\frac{\alpha}{2}+1}\right) \otimes \underline{f}_{y, i j}\right] \underline{\nu}\right\|_{\frac{1}{2}, \partial_{k} \Omega}\right] \\
\quad \leq C\left[\left|\omega_{y, \rho}^{\frac{\alpha}{2}+1} \nabla \delta_{y}^{h}\right|_{0}+\left|\omega_{y, \rho}^{\frac{\alpha}{2}} \nabla \underline{f}_{y, i j}\right|_{0}+\left|\omega_{y, \rho}^{\frac{\alpha}{2}-1} \underline{f}_{y, i j}\right|_{0}\right] .
\end{gathered}
$$


It follows from (2.33b), 2.45), (2.33a) and (2.42) that

$$
\begin{aligned}
\left|\omega_{y, \rho}^{\frac{\alpha}{2}} \nabla \underline{f}_{y, i j}\right|_{0} & \leq C\left[\left|\omega_{y, \rho}^{\frac{\alpha}{2}} \underline{f}_{y, i j}\right|_{1}+\left|\nabla\left(\omega_{y, \rho}^{\frac{\alpha}{2}}\right) \underline{f}_{y, i j}\right|_{0}\right] \\
& \leq C\left[\left|\underline{\underline{\mathcal{E}}}\left(\omega_{y, \rho}^{\frac{\alpha}{2}} \underline{f}_{y, i j}\right)\right|_{0}+\left|\omega_{y, \rho}^{\frac{\alpha}{2}-1} \underline{f}_{y, i j}\right|_{0}\right] \\
& \leq C\left[\left|\omega_{y, \rho}^{\frac{\alpha}{2}} \underline{\underline{\mathcal{E}}}\left(\underline{f}_{y, i j}\right)\right|_{0}+\left|\omega_{y, \rho}^{\frac{\alpha}{2}-1} \underline{f}_{y, i j}\right|_{0}\right] .
\end{aligned}
$$

We have from (2.42) that

$$
\left(\omega_{y, \rho}^{\alpha} \underline{\underline{\mathcal{E}}}\left(\underline{f}_{y, i j}\right), \underline{\underline{\mathcal{E}}}\left(\underline{f}_{y, i j}\right)\right)=\left(\underline{\underline{\mathcal{E}}}\left(\underline{f}_{y, i j}\right), \underline{\underline{\mathcal{E}}}\left(\omega_{y, \rho}^{\alpha} \underline{f}_{y, i j}\right)\right)
$$

$$
-\frac{1}{2}\left(\underline{f}_{y, i j} \otimes \nabla\left(\omega_{y, \rho}^{\alpha}\right)+\nabla\left(\omega_{y, \rho}^{\alpha}\right) \otimes \underline{f}_{y, i j}, \underline{\underline{\mathcal{E}}}\left(\underline{f}_{y, i j}\right)\right) .
$$

Similarly to (2.52), testing (2.30) with $\underline{\eta}=\omega_{y, \rho}^{\alpha} \underline{f}_{y, i j}$ yields that

$$
\left(\underline{\underline{\mathcal{E}}}\left(\underline{f}_{y, i j}\right), \underline{\underline{\mathcal{E}}}\left(\omega_{y, \rho}^{\alpha} \underline{f}_{y, i j}\right)\right)=-\frac{1}{2}\left(\left[\underline{e}_{i} \otimes \underline{e}_{j}+\underline{e}_{j} \otimes \underline{e}_{i}\right] \nabla \delta_{y}^{h}, \omega_{y, \rho}^{\alpha} \underline{f}_{y, i j}\right) .
$$

Combining (2.51), (2.53), (2.54), (2.55) and (2.56) yields that

$$
\max _{k, \ell=1,2} \int_{\Omega} \omega_{y, \rho}^{\alpha+2}\left|\frac{\partial^{2} \underline{f}_{y, i j}}{\partial x_{k} \partial x_{\ell}}\right|^{2} \mathrm{~d} x \leq C(\alpha)\left[\left|\omega_{y, \rho}^{\frac{\alpha}{2}-1} \underline{f}_{y, i j}\right|_{0}^{2}+\left|\omega_{y, \rho}^{\frac{\alpha}{2}+1} \nabla \delta_{y}^{h}\right|_{0}^{2}\right] .
$$

For $p \in\left(1, \frac{2}{\alpha}\right)$, let $\underline{\Upsilon}=N\left(\left(I-P_{\mathrm{RM}}\right) \underline{\xi}, \underline{0}\right)$, where $[\underline{\xi}]_{\ell}=\operatorname{sign}\left(\left[\underline{f}_{y, i j}\right] \ell\right)\left|\left[\underline{f}_{y, i j}\right]_{\ell}\right|^{2 p-1}$, $\ell=1,2$. It follows from (2.44), (2.39), (2.30), (1.17), (2.40) and (2.32) that

$$
\begin{aligned}
\left|\underline{f}_{y, i j}\right|_{0,2 p}^{2 p} & =\left(\underline{\xi}, \underline{f}_{y, i j}\right)=\left(\left(I-P_{\mathrm{RM}}\right) \underline{\xi}, \underline{f}_{y, i j}\right)=\left(\underline{\underline{\mathcal{E}}}(\underline{\Upsilon}), \underline{\underline{\mathcal{E}}}\left(\underline{f}_{y, i j}\right)\right)=\left(\delta_{y}^{h},[\underline{\underline{\mathcal{E}}}(\underline{\Upsilon})]_{i j}\right) \\
& \leq C\left|\delta_{y}^{h}\right|_{0, \frac{2 p}{p+1}}|\underline{\Upsilon}|_{1, \frac{2 p}{p-1}} \leq C\left|\delta_{y}^{h}\right|_{0, \frac{2 p}{p+1}}\|\underline{\Upsilon}\|_{2, \frac{2 p}{2 p-1}} \\
& \leq C\left|\delta_{y}^{h}\right|_{0, \frac{2 p}{p+1}}\left|\left(I-P_{\mathrm{RM}}\right) \underline{\xi}\right|_{0, \frac{2 p}{2 p-1}} \leq C\left|\delta_{y}^{h}\right|_{0, \frac{2 p}{p+1}}|\underline{\xi}|_{0, \frac{2 p}{2 p-1}} \\
& \leq C\left|\delta_{y}^{h}\right|_{0, \frac{2 p}{p+1}}|\underline{\xi}|_{0,2 p} \leq C\left|\delta_{y}^{h}\right|_{0, \frac{2 p}{p+1}}\left|\underline{f}_{y, i j}\right|_{0,2 p}^{2 p-1} \leq C\left|\delta_{y}^{h}\right|_{0, \frac{2 p}{p+1}}^{2 p} \\
& \leq C\left(\int_{\Omega} \omega_{y, \rho}^{-(\alpha+2) p} \mathrm{~d} x\right)\left|\omega_{y, \rho}^{\frac{\alpha}{2}+1} \delta_{y}^{h}\right|_{0}^{2 p} \leq C(\alpha)(\rho h)^{2-(\alpha+2) p}\left|\omega_{y, \rho}^{\frac{\alpha}{2}+1} \delta_{y}^{h}\right|_{0}^{2 p} .
\end{aligned}
$$

Next we have from (2.32) and (2.58) that for $p \in\left(1, \frac{2}{\alpha}\right)$,

$$
\left|\omega_{y, \rho}^{\frac{\alpha}{2}-1} \underline{f}_{y, i j}\right|_{0}^{2} \leq C\left(\int_{\Omega} \omega_{y, \rho}^{(\alpha-2) p^{\prime}} \mathrm{d} x\right)^{\frac{1}{p^{\prime}}}\left|\underline{f}_{y, i j}\right|_{0,2 p}^{2} \leq C(\alpha)(\rho h)^{\alpha-\frac{2}{p}}\left|\underline{f}_{y, i j}\right|_{0,2 p}^{2},
$$

where $\frac{1}{p}+\frac{1}{p^{\prime}}=1$. Finally, combining (2.57), (2.59), (2.58), (2.29) and (2.32) yields that

$$
\begin{aligned}
\max _{k, \ell=1,2} \int_{\Omega} \omega_{y, \rho}^{\alpha+2}\left|\frac{\partial^{2} \underline{f}_{y, i j}}{\partial x_{k} \partial x_{\ell}}\right|^{2} \mathrm{~d} x & \leq C(\alpha, \rho) h^{-2}\left|\omega_{y, \rho}^{\frac{\alpha}{2}+1} \delta_{y}^{h}\right|_{0}^{2} \leq C(\alpha, \rho) h^{-6}\left|\omega_{y, \rho}^{\frac{\alpha}{2}+1}\right|_{0, \sigma_{y}}^{2} \\
& \leq C(\alpha, \rho) h^{\alpha-2}
\end{aligned}
$$

and hence the desired result (2.50).

We now have a discrete analogue of a result similar to (1.20).

Lemma 2.4. Let the assumptions of Lemma 2.3 hold. Then there exists $\delta_{1} \in(0, \delta)$ and $C\left(c_{0}, m_{\mathcal{C}}, M_{\mathcal{C}}\right) \in \mathbb{R}_{>0}$ such that for all $p \in\left[2,2+\delta_{1}\right]$ and for all $h \in\left(0, h_{0}\right)$,

$$
\left|\underline{\underline{\mathcal{E}}}\left(\underline{z}^{h}\right)\right|_{0, p} \leq C \sup _{\underline{0} \neq \underline{\underline{\chi}} \in \underline{\widehat{\widehat{S}}}^{h}} \frac{\left(c\left(\theta^{h}\right) \mathcal{C} \underline{\underline{\mathcal{E}}}\left(\underline{z}^{h}\right), \underline{\underline{\mathcal{E}}}(\underline{\chi})\right)}{|\underline{\underline{\mathcal{E}}}(\underline{\chi})|_{0, q}} \quad \forall \underline{z}^{h} \in \underline{\widehat{S}}^{h}, \quad \forall \theta^{h} \in K^{h},
$$


where $\frac{1}{p}+\frac{1}{q}=1$.

Proof. The proof is an extension of the approach in [17, §8.6] for a scalar secondorder linear elliptic equation. Similarly to (2.38), it follows from (1.20), (2.27) and (2.28) that for all $p \in[2,2+\delta]$, for all $h \in\left(0, h_{0}\right)$ and for all $\underline{z}^{h} \in \underline{\widehat{S}}^{h}$,

$$
\left|\underline{\underline{\mathcal{E}}}\left(\underline{z}^{h}\right)\right|_{0, p} \leq \beta(p) \widehat{\beta}(q) \sup _{\underline{0} \neq \underline{\underline{\eta} \in \underline{\underline{V}}_{q}}} \frac{\left(\underline{\underline{\mathcal{E}}}\left(\underline{z}^{h}\right), \underline{\underline{\mathcal{E}}}\left(P^{h} \underline{\eta}\right)\right)}{\left|\underline{\underline{\mathcal{E}}}\left(P^{h} \underline{\eta}\right)\right|_{0, q}} \leq(1+\sigma(p)) \sup _{\underline{0} \neq \underline{\underline{\chi}} \in \underline{\widehat{\widehat{S}}}^{h}} \frac{\left(\underline{\underline{\mathcal{E}}}\left(\underline{z}^{h}\right), \underline{\underline{\mathcal{E}}}(\underline{\chi})\right)}{|\underline{\underline{\mathcal{E}}}(\underline{\chi})|_{0, q}},
$$

where $\sigma \in C([2,2+\delta]), \sigma(p) \geq 0$ and $\sigma(p) \rightarrow 0$ as $p \rightarrow 2$. On recalling (1.4) and (1.10) we define $B(\underline{z}, \underline{\eta}):=\left(\left(\mathcal{I}-\frac{1}{M_{\mathcal{C}}} c\left(\theta^{h}\right) \mathcal{C}\right) \underline{\underline{\mathcal{E}}}(\underline{z}), \underline{\underline{\mathcal{E}}}(\underline{\eta})\right)$ for all $\underline{z} \in \underline{\widehat{V}}_{p}, \underline{\eta} \in \underline{\widehat{V}}_{q}$ and $\theta^{h} \in K^{h}$. It follows from (1.10) and (1.4) that

$$
|B(\underline{z}, \underline{\eta})| \leq\left(1-\frac{c_{0} m_{\mathcal{C}}}{M_{\mathcal{C}}}\right)|\underline{\underline{\mathcal{E}}}(\underline{z})|_{0, p}|\underline{\underline{\mathcal{E}}}(\underline{\eta})|_{0, q} .
$$

Combining (2.61) and (2.62) yields for all $\underline{z}^{h} \in \underline{\widehat{S}}^{h}$ and $\theta^{h} \in K^{h}$ that

$$
\left[\frac{1}{1+\sigma(p)}-\left(1-\frac{c_{0} m_{\mathcal{C}}}{M_{\mathcal{C}}}\right)\right]\left|\underline{\underline{\mathcal{E}}}\left(\underline{z}^{h}\right)\right|_{0, p} \leq \frac{1}{M_{\mathcal{C}}} \sup _{\underline{0} \neq \underline{\underline{\chi}} \in \underline{\widehat{S}}^{h}} \frac{\left(c\left(\theta^{h}\right) \mathcal{C} \underline{\underline{\mathcal{E}}}\left(\underline{z}^{h}\right), \underline{\underline{\mathcal{E}}}(\underline{\chi})\right)}{|\underline{\underline{\mathcal{E}}}(\underline{\chi})|_{0, q}} .
$$

Since $\sigma(p) \rightarrow 0$ as $p \rightarrow 2$ and $\sigma$ is continuous, one can choose $\delta_{1} \in(0, \delta)$ such that $\sigma(p) \leq \frac{1}{2} \frac{c_{0} m_{\mathcal{C}}}{M_{\mathcal{C}}-c_{0} m_{\mathcal{C}}}$ for all $p \in\left[2,2+\delta_{1}\right]$. Hence (2.63) yields the result (2.60).

Remark 2.2. It is now straightforward to establish a global $L^{\infty}\left(0, T ; \underline{W}^{1, p}(\Omega)\right)$, $p>2$, bound for $\underline{u}$ solving (1.12e). Let $\theta \in L^{\infty}\left(\Omega_{T}\right)$. Then, similarly to the proof of Lemma 2.4, it follows from (1.19), (1.20), (1.10), (1.4), (1.12e) and a trace inequality that for a.a. $t \in(0, T)$,

$$
\left[\frac{1}{\beta(p)}-\left(1-\frac{c_{0} m_{\mathcal{C}}}{M_{\mathcal{C}}}\right)\right]|\underline{\underline{\mathcal{E}}}(\underline{u}(\cdot, t))|_{0, p} \leq \frac{1}{M_{\mathcal{C}}} \sup _{\underline{0} \neq \underline{\eta} \in \underline{\widehat{V}}_{q}} \frac{\left|\int_{\partial \Omega} \underline{g} \cdot \underline{\eta} \mathrm{d} s\right|}{|\underline{\underline{\mathcal{E}}}(\underline{\eta})|_{0, q}} \leq C \sup _{\underline{0} \neq \underline{\eta} \in \underline{\underline{V}}_{q}} \frac{|\underline{\eta}|_{0,1, \partial \Omega}}{\|\underline{\eta}\|_{1, q}} \leq C .
$$

We introduce for all $\varepsilon \in(0,1), b_{\varepsilon}:[-1,1] \rightarrow[\varepsilon(2-\varepsilon), 1]$ defined by

$$
b_{\varepsilon}(s):=\frac{1}{G_{\varepsilon}^{\prime \prime}(s)} \geq \frac{1}{G^{\prime \prime}(s)}=b(s) .
$$

Then the following lemma follows immediately from the construction of $\Xi_{\varepsilon}$; see [12, Lemmas 2.2 and 2.3] for details.

Lemma 2.5. Let the assumptions $(A)$ hold and let $\|\cdot\|$ denote the spectral norm on $\mathbb{R}^{2 \times 2}$. Then for any given $\varepsilon \in(0,1)$ the function $\Xi_{\varepsilon}: S^{h} \rightarrow\left[L^{\infty}(\Omega)\right]^{2 \times 2}$ satisfies for all $z^{h} \in K^{h}, \underline{\xi} \in \mathbb{R}^{2}$ and for all $\sigma \in \mathcal{T}^{h}$,

$$
\begin{gathered}
\varepsilon(2-\varepsilon) \underline{\xi}^{T} \underline{\xi} \leq \min _{x \in \bar{\sigma}} b_{\varepsilon}\left(z^{h}(x)\right) \underline{\xi}^{T} \underline{\xi} \leq\left.\underline{\xi}^{T} \Xi_{\varepsilon}\left(z^{h}\right)\right|_{\sigma} \underline{\xi} \leq \max _{x \in \bar{\sigma}} b_{\varepsilon}\left(z^{h}(x)\right) \underline{\xi}^{T} \underline{\xi} \leq \underline{\xi}^{T} \underline{\xi}, \\
(2.66 \mathrm{~b}) \quad \max _{x \in \sigma}\left\|\left[\Xi_{\varepsilon}\left(z^{h}\right)-b_{\varepsilon}\left(z^{h}\right) \mathcal{I}\right](x)\right\| \leq h_{\sigma}\left|\nabla\left[b_{\varepsilon}\left(z^{h}\right)\right]\right|_{0, \infty, \sigma} \leq 2 h_{\sigma}\left|\nabla z^{h}\right|_{\sigma} \mid .
\end{gathered}
$$

In the remainder of this section, we establish stability bounds for the solution of $(2.3 \mathrm{a}-\mathrm{c})$ that are needed for our convergence analysis in $\$ 3$.

Lemma 2.6. Let the assumptions $(A)$ hold and $\Theta_{\varepsilon}^{n-1} \in K^{h}$. Then for all $\varepsilon \in(0,1)$ and for all $h, \tau_{n}>0$ there exists a solution $\left\{\underline{U}_{\varepsilon}^{n}, \Theta_{\varepsilon}^{n}, W_{\varepsilon}^{n}\right\}$ to the $n$-th step of $\left(\mathrm{P}_{\varepsilon}^{h, \tau}\right)$ 
with $f \Theta_{\varepsilon}^{n}=f \Theta_{\varepsilon}^{n-1} .\left\{\underline{U}_{\varepsilon}^{n}, \Theta_{\varepsilon}^{n}\right\}$ is unique. In addition, $W_{\varepsilon}^{n}$ is unique if there exists $j \in J$ such that $\Theta_{\varepsilon}^{n}\left(p_{j}\right) \in(-1,1)$. Moreover, it follows that

$$
\begin{aligned}
\mathcal{J}\left(\Theta_{\varepsilon}^{n}, \underline{U}_{\varepsilon}^{n}\right)+ & \frac{1}{2}\left[\gamma\left|\Theta_{\varepsilon}^{n}-\Theta_{\varepsilon}^{n-1}\right|_{1}^{2}+\gamma^{-1}\left|\Theta_{\varepsilon}^{n}-\Theta_{\varepsilon}^{n-1}\right|_{h}^{2}\right] \\
& +\frac{1}{2} \gamma^{-1} \tau_{n}\left(\Xi_{\varepsilon}\left(\Theta_{\varepsilon}^{n-1}\right) \nabla W_{\varepsilon}^{n}, \nabla W_{\varepsilon}^{n}\right) \leq \mathcal{J}\left(\Theta_{\varepsilon}^{n-1}, \underline{U}_{\varepsilon}^{n}\right),
\end{aligned}
$$

where

$\mathcal{J}\left(\Theta_{\varepsilon}^{n}, \underline{U}_{\varepsilon}^{n}\right):=\frac{1}{2}\left[\gamma\left|\Theta_{\varepsilon}^{n}\right|_{1}^{2}-\gamma^{-1}\left|\Theta_{\varepsilon}^{n}\right|_{h}^{2}\right]+\left[\int_{\Omega} E\left(\Theta_{\varepsilon}^{n}, \underline{U}_{\varepsilon}^{n}\right) \mathrm{d} x-\int_{\partial \Omega} \underline{g} \cdot \underline{U}_{\varepsilon}^{n} \mathrm{~d} s\right] \geq \mathcal{J}_{0}$,

with $\mathcal{J}_{0} \in \mathbb{R}$. Furthermore it follows that

$$
\begin{aligned}
& \gamma^{2}\left(G_{\varepsilon}\left(\Theta_{\varepsilon}^{n}\right)-G_{\varepsilon}\left(\Theta_{\varepsilon}^{n-1}\right), 1\right)^{h}+\frac{\gamma^{2}}{2} \tau_{n}\left|\Delta^{h} \Theta_{\varepsilon}^{n}\right|_{h}^{2} \\
& \leq \varepsilon^{-1} \gamma^{2}\left|\Theta_{\varepsilon}^{n}-\Theta_{\varepsilon}^{n-1}\right|_{h}^{2}+\gamma \tau_{n}\left(\nabla W_{\varepsilon}^{n}, \nabla\left[\Theta_{\varepsilon}^{n}-\Theta_{\varepsilon}^{n-1}\right]\right) \\
&+\tau_{n}\left[\left(\nabla \Theta_{\varepsilon}^{n}, \nabla \Theta_{\varepsilon}^{n-1}\right)+C\left|\underline{\underline{\mathcal{E}}}\left(\underline{U}_{\varepsilon}^{n}\right)\right|_{0,4}^{4}\right] .
\end{aligned}
$$

Proof. As (2.3a) is a linear finite-dimensional system, existence of $\underline{U}_{\varepsilon}^{n}$ follows from uniqueness. Given $\Theta_{\varepsilon}^{n-1} \in K^{h}$, it follows from (1.4), (1.10) and (1.19) that

$\left(c\left(\Theta_{\varepsilon}^{n-1}\right) \mathcal{C} \underline{\underline{\mathcal{E}}}(\underline{U}), \underline{\underline{\mathcal{E}}}(\underline{U})\right) \geq c_{0}(\mathcal{C} \underline{\underline{\mathcal{E}}}(\underline{U}), \underline{\underline{\mathcal{E}}}(\underline{U})) \geq c_{0} m_{\mathcal{C}}|\underline{\underline{\mathcal{E}}}(\underline{U})|_{0}^{2} \geq C\|\underline{U}\|_{1}^{2} \quad \forall \underline{U} \in \underline{\widehat{S}}^{h}$.

Hence we have existence and uniqueness of $\underline{U}_{\varepsilon}^{n} \in \underline{\widehat{S}}^{h}$ solving (2.3a). The existence and uniqueness results on $\left\{\Theta_{\varepsilon}^{n}, W_{\varepsilon}^{n}\right\} \in K^{h} \times S^{h}$ can be shown with the techniques in [12, Lemma 2.4]. The details are omitted here for the sake of brevity.

It follows from (1.3), (1.10), (1.13), a trace inequality and (1.19) that

$$
\begin{aligned}
\mathcal{J}\left(\Theta_{\varepsilon}^{n}, \underline{U}_{\varepsilon}^{n}\right) & \geq-\frac{1}{2} \gamma^{-1} \underline{m}(\Omega)+\left[\int_{\Omega} E\left(\Theta_{\varepsilon}^{n}, \underline{U}_{\varepsilon}^{n}\right) \mathrm{d} x-\int_{\partial \Omega} \underline{g} \cdot \underline{U_{\varepsilon}^{n}} \mathrm{~d} s\right] \\
& \geq-\frac{1}{2} \gamma^{-1} \underline{m}(\Omega)+\frac{1}{2} c_{0} m_{\mathcal{C}}\left|\underline{\underline{\mathcal{E}}}\left(\underline{U}_{\varepsilon}^{n}\right)\right|_{0}^{2}-\|\underline{g}\|_{0, \infty, \partial \Omega}\left\|\underline{U}_{\varepsilon}^{n}\right\|_{0,1, \partial \Omega} \\
& \geq-\frac{1}{2} \gamma^{-1} \underline{\underline{m}}(\Omega)+\frac{1}{2} c_{0} m_{\mathcal{C}}\left|\underline{\underline{\mathcal{E}}}\left(\underline{U_{\varepsilon}^{n}}\right)\right|_{0}^{2}-C\left|\underline{\underline{\mathcal{E}}}\left(\underline{U}_{\varepsilon}^{n}\right)\right|_{0} \geq \mathcal{J}_{0} .
\end{aligned}
$$

Furthermore, choosing $\chi \equiv W_{\varepsilon}^{n}$ in (2.3b), $\chi \equiv \Theta_{\varepsilon}^{n-1}$ in (2.3c) and noting the fact that $c^{\prime}\left(\Theta_{\varepsilon}^{n-1}\right)\left[\Theta_{\varepsilon}^{n}-\Theta_{\varepsilon}^{n-1}\right]=c\left(\Theta_{\varepsilon}^{n}\right)-c\left(\Theta_{\varepsilon}^{n-1}\right)$, as $c$ is affine linear, and the elementary identity $2 r(r-s)=\left(r^{2}-s^{2}\right)+(r-s)^{2}$, it follows from (1.3) and (2.69) that the desired results (2.67a, b) hold.

Choosing $\chi \equiv \pi^{h}\left[G_{\varepsilon}^{\prime}\left(\Theta_{\varepsilon}^{n-1}\right)\right]$ in (2.3b) , and noting (2.2) yields that

$$
\gamma\left(\Theta_{\varepsilon}^{n}-\Theta_{\varepsilon}^{n-1}, G_{\varepsilon}^{\prime}\left(\Theta_{\varepsilon}^{n-1}\right)\right)^{h}+\tau_{n}\left(\nabla W_{\varepsilon}^{n}, \nabla \Theta_{\varepsilon}^{n-1}\right)=0 .
$$

We now apply an argument similar to that in [6. Theorem 2.3]. From (2.3c) we have for all $j \in J$ on choosing $\chi \equiv \Theta_{\varepsilon}^{n}+\varsigma \chi_{j}, \Theta_{\varepsilon}^{n} \pm \varsigma \chi_{j}, \Theta_{\varepsilon}^{n}-\varsigma \chi_{j} \in K^{h}$, respectively for $\varsigma>0$ sufficiently small, that

$$
\begin{array}{r}
\gamma\left(\nabla \Theta_{\varepsilon}^{n}, \nabla \chi_{j}\right)-\left(W_{\varepsilon}^{n}+\gamma^{-1} \Theta_{\varepsilon}^{n-1}, \chi_{j}\right)^{h}+\frac{1}{2}\left(c^{\prime}\left(\Theta_{\varepsilon}^{n-1}\right) \mathcal{C} \underline{\underline{\mathcal{E}}}\left(\underline{U}_{\varepsilon}^{n}\right): \underline{\underline{\mathcal{E}}}\left(\underline{U}_{\varepsilon}^{n}\right), \chi_{j}\right) \\
\left\{\begin{array} { l } 
{ \geq 0 } \\
{ = 0 } \\
{ \leq 0 }
\end{array} \quad \text { if } \Theta _ { \varepsilon } ^ { n } ( p _ { j } ) \left\{\begin{array}{l}
=-1, \\
\in(-1,1), \\
=1 .
\end{array}\right.\right.
\end{array}
$$

From (2.14) and (2.1) it follows for all $j \in J$ that

$$
\Theta_{\varepsilon}^{n}\left(p_{j}\right)= \pm 1 \Longrightarrow \pm \Theta_{\varepsilon}^{n}\left(p_{j}\right) \geq \pm \Theta_{\varepsilon}^{n}\left(p_{i}\right) \quad \forall i \in J \Longrightarrow \pm \Delta^{h} \Theta_{\varepsilon}^{n}\left(p_{j}\right) \leq 0
$$


Combining (2.71) and (2.72), and noting (2.14), (1.10) and (2.8), yields that

$$
\begin{aligned}
\gamma^{2}\left|\Delta^{h} \Theta_{\varepsilon}^{n}\right|_{h}^{2} & =-\gamma^{2}\left(\nabla \Theta_{\varepsilon}^{n}, \nabla\left(\Delta^{h} \Theta_{\varepsilon}^{n}\right)\right) \\
& \leq-\left(\gamma W_{\varepsilon}^{n}+\Theta_{\varepsilon}^{n-1}, \Delta^{h} \Theta_{\varepsilon}^{n}\right)^{h}+\frac{\gamma}{2}\left(c^{\prime}\left(\Theta_{\varepsilon}^{n-1}\right) \mathcal{C} \underline{\underline{\mathcal{E}}}\left(\underline{U}_{\varepsilon}^{n}\right): \underline{\underline{\mathcal{E}}}\left(\underline{U}_{\varepsilon}^{n}\right), \Delta^{h} \Theta_{\varepsilon}^{n}\right) \\
& \leq\left(\nabla\left[\gamma W_{\varepsilon}^{n}+\Theta_{\varepsilon}^{n-1}\right], \nabla \Theta_{\varepsilon}^{n}\right)+\frac{\gamma^{2}}{2}\left|\Delta^{h} \Theta_{\varepsilon}^{n}\right|_{h}^{2}+C\left|\underline{\underline{\mathcal{E}}}\left(\underline{U}_{\varepsilon}^{n}\right)\right|_{0,4}^{4} .
\end{aligned}
$$

It follows from (2.70), [12, (2.8a)] and (2.73) that

$$
\begin{gathered}
\gamma^{2}\left(G_{\varepsilon}\left(\Theta_{\varepsilon}^{n}\right)-G_{\varepsilon}\left(\Theta_{\varepsilon}^{n-1}\right), 1\right)^{h}+\frac{\gamma^{2}}{2} \tau_{n}\left|\Delta^{h} \Theta_{\varepsilon}^{n}\right|_{h}^{2} \\
\leq \gamma^{2}\left(\Theta_{\varepsilon}^{n}-\Theta_{\varepsilon}^{n-1}, G_{\varepsilon}^{\prime}\left(\Theta_{\varepsilon}^{n}\right)\right)^{h}+\tau_{n}\left(\nabla\left[\gamma W_{\varepsilon}^{n}+\Theta_{\varepsilon}^{n-1}\right], \nabla \Theta_{\varepsilon}^{n}\right)+C \tau_{n}\left|\underline{\underline{\mathcal{E}}}\left(\underline{U}_{\varepsilon}^{n}\right)\right|_{0,4}^{4} \\
\leq \gamma^{2}\left(\Theta_{\varepsilon}^{n}-\Theta_{\varepsilon}^{n-1}, G_{\varepsilon}^{\prime}\left(\Theta_{\varepsilon}^{n}\right)-G_{\varepsilon}^{\prime}\left(\Theta_{\varepsilon}^{n-1}\right)\right)^{h}+\tau_{n} \gamma\left(\nabla W_{\varepsilon}^{n}, \nabla\left[\Theta_{\varepsilon}^{n}-\Theta_{\varepsilon}^{n-1}\right]\right) \\
+\tau_{n}\left(\nabla \Theta_{\varepsilon}^{n}, \nabla \Theta_{\varepsilon}^{n-1}\right)+C \tau_{n}\left|\underline{\underline{\mathcal{E}}}\left(\underline{U}_{\varepsilon}^{n}\right)\right|_{0,4}^{4} \\
\leq \varepsilon^{-1} \gamma^{2}\left|\Theta_{\varepsilon}^{n}-\Theta_{\varepsilon}^{n-1}\right|_{h}^{2}+\tau_{n}\left[\gamma \left(\nabla W_{\varepsilon}^{n},\right.\right. \\
\left.\nabla\left[\Theta_{\varepsilon}^{n}-\Theta_{\varepsilon}^{n-1}\right]\right) \\
\left.+\left(\nabla \Theta_{\varepsilon}^{n}, \nabla \Theta_{\varepsilon}^{n-1}\right)+C\left|\underline{\underline{E}}\left(\underline{U}_{\varepsilon}^{n}\right)\right|_{0,4}^{4}\right]
\end{gathered}
$$

and hence the desired result (2.68).

Remark 2.3. We note that $(2.67 \mathrm{a}, \mathrm{b})$ and $(2.68)$ are the discrete analogues of the energy estimates (1.15) and (1.16), respectively.

Lemma 2.7. Let the assumptions of Lemmas 2.4 and 2.6 hold. Then for all $p \in\left[2,2+\delta_{1}\right]$ and for all $h \in\left(0, h_{0}\right)$,

$$
\left.\underline{\underline{\mathcal{E}}}\left(\underline{U}_{\varepsilon}^{n}\right)\right|_{0, p} \leq C .
$$

Proof. Similarly to (2.64), it follows from (2.60), (2.3a), (1.13), (1.19) and a trace inequality that

$$
\left|\underline{\underline{\mathcal{E}}}\left(\underline{U}_{\varepsilon}^{n}\right)\right|_{0, p} \leq C \sup _{\underline{0} \neq \underline{\chi} \in \underline{\widehat{S}}^{h}} \frac{\left|\int_{\partial \Omega} \underline{g} \cdot \underline{\chi} \mathrm{d} s\right|}{|\underline{\underline{\mathcal{E}}}(\underline{\chi})|_{0, q}} \leq C \sup _{\underline{0} \neq \underline{\chi} \in \underline{\widehat{S}}^{h}} \frac{|\underline{\chi}|_{0,1, \partial \Omega}}{|\underline{\underline{\mathcal{E}}}(\underline{\chi})|_{0, q}} \leq C \sup _{\underline{0} \neq \underline{\chi} \in \underline{\widehat{S}}^{h}} \frac{|\underline{\chi}|_{0,1, \partial \Omega}}{\|\underline{\chi}\|_{1, q}} \leq C
$$

and hence the desired result (2.74).

On recalling (1.13), we set

$$
\underline{\widetilde{U}}_{\varepsilon}^{n}:=\underline{U}_{\varepsilon}^{n}-\underline{\underline{S}}^{*} \underline{x}
$$

as it is easier, by exploiting (2.25), to bound $\left|L^{h} \widetilde{U}_{\varepsilon}^{n}\right|_{0}$ than to bound $\left|L^{h} \underline{U}_{\varepsilon}^{n}\right|_{0}$; see the lemma below.

Lemma 2.8. Let the assumptions of Lemmas 2.2 and 2.7 hold. Assuming that $\Theta_{\varepsilon}^{n-1}=1$ on $\partial \Omega$, it follows that

$$
\left|L^{h}\left(\underline{\widetilde{U}}_{\varepsilon}^{n}\right)\right|_{0} \leq C\left|\Theta_{\varepsilon}^{n-1}\right|_{1,4}^{2} .
$$

Moreover, for all $h \in\left(0, h_{0}\right)$,

$$
\left|\underline{\underline{\mathcal{E}}}\left(\underline{U}_{\varepsilon}^{n}\right)\right|_{0,4}^{4} \leq C\left(\delta_{1}\right)\left[\left|\Theta_{\varepsilon}^{n-1}\right|_{1,4}^{4-\delta_{1}}+1\right] .
$$

Proof. For ease of notation, let $c^{n-1}:=c\left(\Theta_{\varepsilon}^{n-1}\right)$. Assuming that $\Theta_{\varepsilon}^{n-1}=1$ on $\partial \Omega$, it follows from (1.13) and $\underline{\underline{\mathcal{E}}}\left(\underline{\underline{S}}^{*} \underline{x}\right)=\underline{\underline{S}}^{*} \underline{\underline{\mathcal{E}}}(\underline{x})=\underline{\underline{S}}^{*}$ that

$$
\begin{aligned}
\int_{\partial \Omega} \underline{g} \cdot \underline{\eta} \mathrm{d} s & =\int_{\partial \Omega} c^{n-1}\left(\mathcal{C} \underline{\underline{S}}^{*} \underline{\nu}\right) \cdot \underline{\eta} \mathrm{d} s=\left(\nabla \cdot\left(c^{n-1} \mathcal{C} \underline{\underline{S}}^{*} \underline{\eta}\right), 1\right) \\
& \left.\left.=\left(c^{n-1} \mathcal{C} \underline{\underline{\mathcal{E}}} \underline{\underline{S}}^{*} \underline{x}\right), \underline{\underline{\mathcal{E}}} \underline{\eta}\right)\right)+\left(\nabla \cdot\left(c^{n-1} \mathcal{C} \underline{\underline{S}}^{*}\right), \underline{\eta}\right) \quad \forall \underline{\eta} \in \underline{H}^{1}(\Omega) .
\end{aligned}
$$


Combining (2.3a), (2.75), and (2.78) yields that

$$
\left(c^{n-1} \mathcal{C} \underline{\underline{\mathcal{E}}}\left(\underline{\widetilde{U}}_{\varepsilon}^{n}\right), \underline{\underline{\mathcal{E}}}(\underline{\chi})\right)=\left(\nabla \cdot\left(c^{n-1} \underline{\underline{S}}\right), \underline{\chi}\right) \quad \forall \underline{\chi} \in \underline{S}^{h} .
$$

For the ensuing analysis it is convenient to introduce $\underline{\tilde{u}}_{\varepsilon}^{n} \in \underline{\underline{H}}^{1}(\Omega)$ such that

$$
\left(c^{n-1} \mathcal{C} \underline{\underline{\mathcal{E}}}\left(\underline{\tilde{u}_{\varepsilon}^{n}}\right), \underline{\underline{\mathcal{E}}}(\underline{\eta})\right)=\left(\nabla \cdot\left(c^{n-1} \underline{\underline{S}}\right), \underline{\eta}\right) \quad \forall \underline{\eta} \in \underline{H}^{1}(\Omega) .
$$

Existence and uniqueness of $\tilde{u}_{\varepsilon}^{n}$, and the bound

$$
\left\|\underline{\tilde{u}}_{\varepsilon}^{n}\right\|_{1} \leq C
$$

are easily established on noting (2.78), (1.13), (1.4), (1.10), (1.19) and a trace inequality.

We now address the $H^{2}(\Omega)$ regularity of $\underline{\tilde{u}}_{\varepsilon}^{n}$. If $\widehat{\underline{\eta}} \in \underline{H}^{1}(\Omega)$, then $\underline{\eta}:=\left[c^{n-1}\right]^{-1} \widehat{\widehat{\eta}}$ satisfies, on noting (1.4) and (1.17),

$$
|\underline{\eta}|_{1} \leq C\left[|\underline{\underline{\eta}}|_{1}+\left|\Theta_{\varepsilon}^{n-1}\right|_{1,2+\varsigma}|\underline{\underline{\eta}}|_{0, \frac{2(2+\varsigma)}{\varsigma}}\right] \leq C\left[1+\left|\Theta_{\varepsilon}^{n-1}\right|_{1,2+\varsigma}\right]\|\underline{\hat{p}}\|_{1}, \quad \varsigma>0,
$$

and hence $\underline{\eta} \in \underline{H}^{1}(\Omega)$. Choosing $\underline{\eta} \equiv\left[c^{n-1}\right]^{-1} \widehat{\widehat{\eta}}$ in (2.80) yields, on noting (2.42), that for all $\underline{\hat{\eta}} \in \underline{H}^{1}(\Omega)$,

$$
\left(\mathcal{C} \underline{\underline{\mathcal{E}}}\left(\underline{\tilde{u}}_{\varepsilon}^{n}\right), \underline{\underline{\mathcal{E}}}(\underline{\widehat{\eta}})\right)=\left(\left[c^{n-1}\right]^{-1} \nabla \cdot\left(c^{n-1} \underline{\underline{S}}\right)-c^{n-1} \mathcal{C} \underline{\underline{\mathcal{E}}}\left(\underline{\tilde{u}}_{\varepsilon}^{n}\right) \nabla\left[c^{n-1}\right]^{-1}, \underline{\widehat{\eta}}\right) .
$$

It follows from (2.82), (2.23), (1.17), (2.81) and (1.4) that

$$
\begin{aligned}
\left\|\underline{\tilde{u}}_{\varepsilon}^{n}\right\|_{2} & \leq C\left[\left|\underline{\underline{\mathcal{E}}}\left(\tilde{u}_{\varepsilon}^{n}\right)\right|_{0,4}\left|c^{n-1}\right|_{1,4}+\left|c^{n-1}\right|_{1}\right] \leq C\left[\left|\underline{\underline{u}}_{\varepsilon}^{n}\right|_{1}^{\frac{1}{2}}\left\|\underline{\tilde{u}}_{\varepsilon}^{n}\right\|_{2}^{\frac{1}{2}}+1\right]\left|c^{n-1}\right|_{1,4} \\
(2.83) & \leq C\left|c^{n-1}\right|_{1,4}^{2} \leq C\left|\Theta_{\varepsilon}^{n-1}\right|_{1,4}^{2} .
\end{aligned}
$$

From (1.10), (1.4), (1.19), (2.80), (2.79), (1.9), (2.6) and (2.83) we have that

$$
\begin{aligned}
C_{1}\left\|\underline{\tilde{u}}_{\varepsilon}^{n}-\underline{\widetilde{U}}_{\varepsilon}^{n}\right\|_{1}^{2} & \leq\left(c^{n-1} \mathcal{C} \underline{\underline{\mathcal{E}}}\left(\underline{\underline{u}}_{\varepsilon}^{n}-\underline{\widetilde{U}}_{\varepsilon}^{n}\right), \underline{\underline{\mathcal{E}}}\left(\underline{\underline{u}}_{\varepsilon}^{n}-\underline{\widetilde{U}}_{\varepsilon}^{n}\right)\right) \\
& \leq\left(c^{n-1} \mathcal{C} \underline{\underline{\mathcal{E}}}\left(\left(I-\pi^{h}\right) \underline{\tilde{u}}_{\varepsilon}^{n}\right), \underline{\underline{\mathcal{E}}}\left(\left(I-\pi^{h}\right) \underline{\tilde{u}}_{\varepsilon}^{n}\right)\right) \\
& \leq C_{2}\left|\left(I-\pi^{h}\right) \underline{\tilde{u}}_{\varepsilon}^{n}\right|_{1}^{2} \leq C_{3} h^{2}\left|\underline{\tilde{u}}_{\varepsilon}^{n}\right|_{2}^{2} .
\end{aligned}
$$

It follows from (2.24) and (2.84) that

$$
\begin{aligned}
& \left|L^{h} \underline{\widetilde{U}}_{\varepsilon}^{n}\right|_{0} \leq\left|L^{h}\left(\underline{\widetilde{U}}_{\varepsilon}^{n}-\pi^{h} \underline{\tilde{u}}_{\varepsilon}^{n}\right)\right|_{0}+\left|L^{h}\left(\pi^{h} \underline{\tilde{u}}_{\varepsilon}^{n}\right)\right|_{0} \leq C h^{-1}\left|\underline{\widetilde{U}}_{\varepsilon}^{n}-\pi^{h} \underline{\tilde{u}}_{\varepsilon}^{n}\right|_{1}+\left|L^{h}\left(\pi^{h} \underline{\tilde{u}}_{\varepsilon}^{n}\right)\right|_{0} \\
& \quad(2.85) \\
& \quad \leq C h^{-1}\left[\left|\underline{\widetilde{U}}_{\varepsilon}^{n}-\underline{\tilde{u}}_{\varepsilon}^{n}\right|_{1}+\left|\left(I-\pi^{h}\right) \underline{\tilde{u}}_{\varepsilon}^{n}\right|_{1}\right]+\left|L^{h}\left(\pi^{h} \underline{\tilde{u}}_{\varepsilon}^{n}\right)\right|_{0} \leq C\left|\underline{\tilde{u}}_{\varepsilon}^{n}\right|_{2}+\left|L^{h}\left(\pi^{h} \underline{\tilde{u}}_{\varepsilon}^{n}\right)\right|_{0} .
\end{aligned}
$$

It follows from (2.80) and (1.4) that

$$
c^{n-1} \mathcal{C} \underline{\underline{\mathcal{E}}}\left(\underline{\tilde{u}}_{\varepsilon}^{n}\right) \underline{\nu}=\underline{0} \quad \text { on } \partial \Omega \quad \Longrightarrow \quad \mathcal{C} \underline{\underline{\mathcal{E}}}\left(\underline{\tilde{u}}_{\varepsilon}^{n}\right) \underline{\nu}=\underline{0} \quad \text { on } \partial \Omega .
$$

The desired result (2.76) then follows from (2.85), (2.86), (2.25) and (2.83).

It follows from (2.75) that

$$
\left|\underline{\underline{\mathcal{E}}}\left(\underline{U}_{\varepsilon}^{n}\right)\right|_{0,4}^{4} \leq C\left[\left|\underline{\underline{\mathcal{E}}}\left(\widetilde{\widetilde{U}}_{\varepsilon}^{n}\right)\right|_{0,4}^{4}+1\right] .
$$

On noting (2.24), we have for any $\alpha \in(0,1)$ that

$$
\left|\underline{\underline{\mathcal{E}}}\left(\underline{\widetilde{U}}_{\varepsilon}^{n}\right)\right|_{0,4}^{4} \leq C(\alpha)\left|\underline{\underline{\mathcal{E}}}\left(\underline{\widetilde{U}}_{\varepsilon}^{n}\right)\right|_{0,2+2 \alpha}^{2+\alpha}\left|L^{h} \underline{\widetilde{U}}_{\varepsilon}^{n}\right|_{0}^{2-\alpha} .
$$

Combining (2.87), (2.88), (2.75), (2.74) and (2.76) yields the result (2.77). 
Lemma 2.9. Let $\theta^{0} \in K \cap H^{2}(\Omega)$ with $\frac{\partial \theta^{0}}{\partial \nu}=0$ on $\partial \Omega$, and the assumptions ( $A$ ) hold. On choosing $\Theta_{\varepsilon}^{0} \equiv \pi^{h} \theta^{0}$ it follows that $\Theta_{\varepsilon}^{0} \in K^{h}$ is such that for all $h>0$,

$$
\left\|\Theta_{\varepsilon}^{0}\right\|_{1}^{2}+\left|\Delta^{h} \Theta_{\varepsilon}^{0}\right|_{h}^{2}+\left(G_{\varepsilon}\left(\Theta_{\varepsilon}^{0}\right), 1\right)^{h} \leq C .
$$

Proof. The desired result (2.89) follows from (2.6), (2.17) and (2.8).

Theorem 2.1. Let the assumptions of Lemma 2.9 hold. Then for all $\varepsilon \in(0,1)$, $h \in\left(0, h_{0}\right)$ and for all time partitions $\left\{\tau_{n}\right\}_{n=1}^{N}$, the solution $\left\{\underline{U}_{\varepsilon}^{n}, \Theta_{\varepsilon}^{n}, W_{\varepsilon}^{n}\right\}_{n=1}^{N}$ to $\left(\mathrm{P}_{\varepsilon}^{h, \tau}\right)$ is such that $f \Theta_{\varepsilon}^{n}=f \Theta_{\varepsilon}^{0}, n=1 \rightarrow N$, and

$$
\begin{aligned}
\gamma \max _{n=1 \rightarrow N}\left\|\Theta_{\varepsilon}^{n}\right\|_{1}^{2}+\sum_{n=1}^{N}\left[\gamma\left|\Theta_{\varepsilon}^{n}-\Theta_{\varepsilon}^{n-1}\right|_{1}^{2}+\gamma^{-1} \tau_{n}\left(\Xi_{\varepsilon}\left(\Theta_{\varepsilon}^{n-1}\right) \nabla W_{\varepsilon}^{n}, \nabla W_{\varepsilon}^{n}\right)\right] \\
+\sum_{n=2}^{N}\left(c\left(\Theta_{\varepsilon}^{n-1}\right) \mathcal{C} \underline{\underline{\mathcal{E}}}\left(\underline{U_{\varepsilon}^{n}}-\underline{U}_{\varepsilon}^{n-1}\right), \underline{\underline{\mathcal{E}}}\left(\underline{U}_{\varepsilon}^{n}-\underline{U}_{\varepsilon}^{n-1}\right)\right) \leq C\left[1+\left\|\Theta_{\varepsilon}^{0}\right\|_{1}^{2}\right] \leq C .
\end{aligned}
$$

In addition,

$$
\gamma \sum_{n=1}^{N} \tau_{n}\left|\mathcal{G}\left[\frac{\Theta_{\varepsilon}^{n}-\Theta_{\varepsilon}^{n-1}}{\tau_{n}}\right]\right|_{1}^{2}+\gamma \tau^{-\frac{1}{2}} \sum_{n=1}^{N}\left|\Theta_{\varepsilon}^{n}-\Theta_{\varepsilon}^{n-1}\right|_{0}^{2} \leq C\left[1+\left\|\Theta_{\varepsilon}^{0}\right\|_{1}^{2}\right] \leq C
$$

Moreover, on assuming (2.23) holds, if $\mathcal{C}$ is anisotropic, $\tau_{n} \leq C \tau_{n-1}, n=2 \rightarrow N$, and $\Theta_{\varepsilon}^{n-1}=1$ on $\partial \Omega, n=1 \rightarrow N$, then

$$
\gamma^{2} \max _{n=1 \rightarrow N}\left(G_{\varepsilon}\left(\Theta_{\varepsilon}^{n}\right), 1\right)^{h}+\sum_{n=1}^{N} \tau_{n}\left[\gamma^{2}\left|\Delta^{h} \Theta_{\varepsilon}^{n}\right|_{h}^{2}+\left\|\underline{U}_{\varepsilon}^{n}\right\|_{1,4}^{4}\right] \leq C(T)\left[1+\varepsilon^{-1} \tau^{\frac{1}{2}}\right]
$$

Proof. First, it follows from (2.67b), (1.3) and (2.3a) that for $n=2 \rightarrow N$, $(2.93)$

$$
\mathcal{J}\left(\Theta_{\varepsilon}^{n-1}, \underline{U}_{\varepsilon}^{n}\right)=\mathcal{J}\left(\Theta_{\varepsilon}^{n-1}, \underline{U}_{\varepsilon}^{n-1}\right)-\frac{1}{2}\left(c\left(\Theta_{\varepsilon}^{n-1}\right) \mathcal{C} \underline{\underline{\mathcal{E}}}\left(\underline{U}_{\varepsilon}^{n}-\underline{U}_{\varepsilon}^{n-1}\right), \underline{\underline{\mathcal{E}}}\left(\underline{U}_{\varepsilon}^{n}-\underline{U}_{\varepsilon}^{n-1}\right)\right) .
$$

Summing (2.67a) from $n=1 \rightarrow k$ and noting (2.93), (2.67b), (1.3), (1.10), (1.4) and a trace inequality, yields for $k=2 \rightarrow N$ that

$$
\begin{aligned}
\mathcal{J}\left(\Theta_{\varepsilon}^{k}, \underline{U}_{\varepsilon}^{k}\right)+ & \frac{1}{2} \sum_{n=1}^{k}\left[\gamma\left|\Theta_{\varepsilon}^{n}-\Theta_{\varepsilon}^{n-1}\right|_{1}^{2}+\gamma^{-1} \tau_{n}\left(\Xi_{\varepsilon}\left(\Theta_{\varepsilon}^{n-1}\right) \nabla W_{\varepsilon}^{n}, \nabla W_{\varepsilon}^{n}\right)\right] \\
& +\frac{1}{2} \sum_{n=2}^{k}\left(c\left(\Theta_{\varepsilon}^{n-1}\right) \mathcal{C} \underline{\underline{\mathcal{E}}}\left(\underline{U}_{\varepsilon}^{n}-\underline{U}_{\varepsilon}^{n-1}\right), \underline{\underline{\mathcal{E}}}\left(\underline{U}_{\varepsilon}^{n}-\underline{U}_{\varepsilon}^{n-1}\right)\right) \\
\leq & \mathcal{J}\left(\Theta_{\varepsilon}^{0}, \underline{U}_{\varepsilon}^{1}\right) \leq C\left[1+\left\|\Theta_{\varepsilon}^{0}\right\|_{1}+\left\|\underline{U}_{\varepsilon}^{1}\right\|_{1}^{2}\right] .
\end{aligned}
$$

The desired result (2.90) then follows from (2.67a) for $n=1$, (2.94) for $k=2 \rightarrow N$, (2.67b), (2.8) and the fact that $\Theta_{\varepsilon}^{n} \in K^{h}, n=0 \rightarrow N$, (2.74), (1.19) and (2.89).

The proof of (2.91) is the same as the proof of [12, (2.43)] and is hence omitted here. Finally, summing (2.68) from $n=1 \rightarrow k$ and noting (2.8), (2.66a), (2.90), (2.91), (2.89), (2.77), (2.16b), our assumption on $\tau_{n}$, and (1.23) yields for any $k \leq N$ 
that

$$
\begin{aligned}
\gamma^{2}\left(G_{\varepsilon}\left(\Theta_{\varepsilon}^{k}\right), 1\right)^{h}+\frac{\gamma^{2}}{2} \sum_{n=1}^{k} \tau_{n}\left|\Delta^{h} \Theta_{\varepsilon}^{n}\right|_{h}^{2} \leq \gamma^{2}\left(G_{\varepsilon}\left(\Theta_{\varepsilon}^{0}\right), 1\right)^{h} \\
\quad+4 \varepsilon^{-1} \gamma^{2} \sum_{n=1}^{k}\left|\Theta_{\varepsilon}^{n}-\Theta_{\varepsilon}^{n-1}\right|_{0}^{2}+t_{k} \max _{n=0 \rightarrow k}\left\|\Theta_{\varepsilon}^{n}\right\|_{1}^{2}+C \sum_{n=1}^{k} \tau_{n}\left|\underline{\underline{\mathcal{E}}}\left(\underline{U_{\varepsilon}^{n}}\right)\right|_{0,4}^{4} \\
\quad+\left[\varepsilon^{-1} \sum_{n=1}^{k} \tau_{n}\left|\left[\Xi_{\varepsilon}\left(\Theta_{\varepsilon}^{n-1}\right)\right]^{\frac{1}{2}} \nabla W_{\varepsilon}^{n}\right|_{0}^{2}\right]^{\frac{1}{2}}\left[\gamma \sum_{n=1}^{k} \tau_{n}\left|\Theta_{\varepsilon}^{n}-\Theta_{\varepsilon}^{n-1}\right|_{1}^{2}\right]^{\frac{1}{2}} \\
\leq C(T)\left[1+\varepsilon^{-1} \tau^{\frac{1}{2}}\right]+C \sum_{n=1}^{k} \tau_{n}\left|\Theta_{\varepsilon}^{n-1}\right|_{1,4}^{4-\delta_{1}} \\
\leq C(T)\left[1+\varepsilon^{-1} \tau^{\frac{1}{2}}\right]+C \sum_{n=2}^{k} \tau_{n-1}\left|\Delta^{h} \Theta_{\varepsilon}^{n-1}\right|_{h}^{2-\frac{\delta_{1}}{2}} \leq C(T)\left[1+\varepsilon^{-1} \tau^{\frac{1}{2}}\right] .
\end{aligned}
$$

Hence the desired result (2.92) follows immediately from (2.95) and (1.19).

\section{Convergence}

In this section we will show convergence of the discrete solutions obtained in Section 2 to a weak solution of problem (P). We will use methods developed in 4, 34] and [12] to deal with the degeneracy of $b$. Furthermore, it will be crucial to show strong convergence of $\underline{\underline{\mathcal{E}}}\left(\underline{U}_{\varepsilon}^{n}\right)$ in order to pass to the limit in the nonlinearity $\mathcal{C} \underline{\underline{\mathcal{E}}}\left(\underline{U_{\varepsilon}^{n}}\right): \underline{\underline{\mathcal{E}}}\left(\underline{U_{\varepsilon}^{n}}\right)$. Let

$$
\begin{array}{ll}
\Theta_{\varepsilon}(\cdot, t):=\frac{t-t_{n-1}}{\tau_{n}} \Theta_{\varepsilon}^{n}(\cdot)+\frac{t_{n}-t}{\tau_{n}} \Theta_{\varepsilon}^{n-1}(\cdot), & t \in\left[t_{n-1}, t_{n}\right], \quad n \geq 1, \\
\Theta_{\varepsilon}^{+}(\cdot, t):=\Theta_{\varepsilon}^{n}(\cdot), \quad \Theta_{\varepsilon}^{-}(\cdot, t):=\Theta_{\varepsilon}^{n-1}(\cdot), \quad t \in\left(t_{n-1}, t_{n}\right], \quad n \geq 1 .
\end{array}
$$

We note for future reference that

$$
\Theta_{\varepsilon}(\cdot, t)-\Theta_{\varepsilon}^{ \pm}(\cdot, t)=\left(t-t_{n}^{ \pm}\right) \frac{\partial \Theta_{\varepsilon}}{\partial t}(\cdot, t), \quad t \in\left(t_{n-1}, t_{n}\right), \quad n \geq 1,
$$

where $t_{n}^{+}:=t_{n}$ and $t_{n}^{-}:=t_{n-1}$. We introduce also $\bar{\tau}(t):=\tau_{n}$ for $t \in\left(t_{n-1}, t_{n}\right]$ and $n \geq 1$.

Using the above notation, and introducing analogous notation for $W_{\varepsilon}$ and $\underline{U}_{\varepsilon}$, $\left(\mathrm{P}_{\varepsilon}^{h, \tau}\right)$ can be restated as: Find $\left\{\underline{U}_{\varepsilon}^{+}, \Theta_{\varepsilon}, W_{\varepsilon}^{+}\right\} \in L^{\infty}\left(0, T ; \underline{\widehat{S}}^{h}\right) \times C\left([0, T] ; K^{h}\right) \times$ $L^{\infty}\left(0, T ; S^{h}\right)$ such that

$$
\begin{array}{cc}
\left.\int_{0}^{T}\left(c\left(\Theta_{\varepsilon}^{-}\right) \mathcal{C} \underline{\underline{\mathcal{E}}}\left(\underline{U}_{\varepsilon}^{+}\right), \underline{\underline{\mathcal{E}}} \underline{\chi}\right)\right) \mathrm{d} t=\int_{0}^{T} \int_{\partial \Omega} \underline{g} \cdot \underline{\chi} \mathrm{d} s \mathrm{~d} t & \forall \underline{\chi} \in L^{\infty}\left(0, T ; \underline{S}^{h}\right), \\
\int_{0}^{T}\left[\gamma\left(\frac{\partial \Theta_{\varepsilon}}{\partial t}, \chi\right)^{h}+\left(\Xi_{\varepsilon}\left(\Theta_{\varepsilon}^{-}\right) \nabla W_{\varepsilon}^{+}, \nabla \chi\right)\right] \mathrm{d} t=0 & \forall \chi \in L^{\infty}\left(0, T ; S^{h}\right), \\
\gamma \int_{0}^{T}\left(\nabla \Theta_{\varepsilon}^{+}, \nabla\left[\chi-\Theta_{\varepsilon}^{+}\right]\right) \mathrm{d} t \geq \int_{0}^{T}\left(W_{\varepsilon}^{+}+\gamma^{-1} \Theta_{\varepsilon}^{-}, \chi-\Theta_{\varepsilon}^{+}\right)^{h} \mathrm{~d} t \\
-\frac{1}{2} \int_{0}^{T}\left(c^{\prime}\left(\Theta_{\varepsilon}^{-}\right) \mathcal{C} \underline{\underline{\mathcal{E}}}\left(\underline{U}_{\varepsilon}^{+}\right): \underline{\underline{\mathcal{E}}}\left(\underline{U}_{\varepsilon}^{+}\right), \chi-\Theta_{\varepsilon}^{+}\right) \mathrm{d} t & \forall \chi \in L^{\infty}\left(0, T ; K^{h}\right) .
\end{array}
$$

Lemma 3.1. Let $\theta^{0} \in K \cap H^{2}(\Omega)$ with $\frac{\partial \theta^{0}}{\partial \nu}=0$ on $\partial \Omega$ and $f \theta^{0} \in(-1,1)$. Let $\left\{\mathcal{T}^{h}, \varepsilon,\left\{\tau_{n}\right\}_{n=1}^{N},\right\}_{h>0}$ be such that $\Omega$ and $\left\{\mathcal{T}^{h}\right\}_{h>0}$ fulfill assumptions $(A), \varepsilon \in(0,1)$ 
with $\varepsilon \rightarrow 0$ as $h \rightarrow 0$ and $\tau_{n} \leq C \tau_{n-1} \leq C \varepsilon^{2}, n=2 \rightarrow N$. Let $\Theta_{\varepsilon}^{0} \equiv \pi^{h} \theta^{0}$. Then there exists a subsequence of $\left\{\underline{U}_{\varepsilon}^{+}, \Theta_{\varepsilon}, W_{\varepsilon}^{+}\right\}_{h}$, where $\left\{\underline{U}_{\varepsilon}^{+}, \Theta_{\varepsilon}, W_{\varepsilon}^{+}\right\}$solve $\left(\mathrm{P}_{\varepsilon}^{h, \tau}\right)$, and functions

(3.4) $\theta \in L^{\infty}(0, T ; K) \cap H^{1}\left(0, T ;\left(H^{1}(\Omega)\right)^{\prime}\right) \quad$ and $\underline{u} \in L^{\infty}\left(0, T ; \widehat{\underline{V}}_{2+\delta_{1}}\right), \delta_{1}>0$, with $\theta(\cdot, 0)=\theta^{0}(\cdot)$ in $L^{2}(\Omega)$ and $f \theta(\cdot, t)=f \theta^{0}$ for a.a. $t \in(0, T)$, such that as $h \rightarrow 0$,

$$
\begin{aligned}
\Theta_{\varepsilon}, \Theta_{\varepsilon}^{ \pm} & \rightarrow \theta & & \text { weak-* in } L^{\infty}\left(0, T ; H^{1}(\Omega)\right), \\
\mathcal{G} \frac{\partial \Theta_{\varepsilon}}{\partial t} & \rightarrow \mathcal{G} \frac{\partial \theta}{\partial t} & & \text { weakly in } L^{2}\left(0, T ; H^{1}(\Omega)\right), \\
\underline{U}_{\varepsilon}^{+} & \rightarrow \underline{u} & & \text { weak-* in } L^{\infty}\left(0, T ; \underline{W}^{1,2+\delta_{1}}(\Omega)\right), \\
\Theta_{\varepsilon}, \Theta_{\varepsilon}^{ \pm} & \rightarrow \theta & & \text { strongly in } L^{2}\left(0, T ; L^{s}(\Omega)\right), \\
\Xi_{\varepsilon}\left(\Theta_{\varepsilon}^{-}\right) & \rightarrow b(\theta) \mathcal{I} & & \text { strongly in } L^{2}\left(0, T ; L^{s}(\Omega)\right), \\
c\left(\Theta_{\varepsilon}^{-}\right) & \rightarrow c(\theta) & & \text { strongly in } L^{2}\left(0, T ; L^{s}(\Omega)\right), \\
\underline{U}_{\varepsilon}^{+} & \rightarrow \underline{u} & & \text { strongly in } L^{2}\left(0, T ; \underline{H}^{1}(\Omega)\right),
\end{aligned}
$$

for all $s \in[2, \infty)$. Moreover, $\{\underline{u}, \theta\}$ satisfy

$$
\int_{\Omega_{T}} c(\theta) \mathcal{C} \underline{\underline{\mathcal{E}}}(\underline{u}): \underline{\underline{\mathcal{E}}}(\underline{\eta}) \mathrm{d} x \mathrm{~d} t=\int_{0}^{T} \int_{\partial \Omega} \underline{g} \cdot \underline{\eta} \mathrm{d} s \mathrm{~d} t \quad \forall \underline{\eta} \in L^{2}\left(0, T ; \underline{H}^{1}(\Omega)\right) .
$$

Furthermore, on assuming (2.23) holds, if $\mathcal{C}$ is anisotropic, and if

$$
\Theta_{\varepsilon}=1 \text { on } \partial \Omega,
$$

then $\{\theta, \underline{u}\}$, in addition to (3.4), satisfy

$$
\theta \in L^{2}\left(0, T ; H^{2}(\Omega)\right) \quad \text { and } \quad \underline{u} \in L^{4}\left(0, T ; \underline{W}^{1,4}(\Omega)\right) ;
$$

and there exists a subsequence of $\left\{\underline{U}_{\varepsilon}^{+}, \Theta_{\varepsilon}, W_{\varepsilon}^{+}\right\}_{h}$ satisfying $(3.5 \mathrm{a}-c)$, (3.6a- $\left.d\right)$ and as $h \rightarrow 0$,

(3.10a) $\Delta^{h} \Theta_{\varepsilon}, \Delta^{h} \Theta_{\varepsilon}^{ \pm} \rightarrow \Delta \theta \quad$ weakly in $L^{2}\left(\Omega_{T}\right)$,

$$
\begin{aligned}
\Theta_{\varepsilon}, \Theta_{\varepsilon}^{ \pm} \rightarrow \theta & \text { weakly in } L^{2}\left(0, T ; W^{1, s}(\Omega)\right), \quad \text { for any } s \in[2, \infty), \\
\Theta_{\varepsilon}, \Theta_{\varepsilon}^{ \pm} \rightarrow \theta & \text { strongly in } L^{2}\left(0, T ; C^{0, \beta}(\bar{\Omega})\right), \quad \text { for any } \beta \in(0,1), \\
\underline{U}_{\varepsilon}^{+} \rightarrow \underline{u} & \text { weakly in } L^{4}\left(0, T ; \underline{W}^{1,4}(\Omega)\right) .
\end{aligned}
$$

Finally, on extracting a further subsequence, it follows for a.a. $t \in(0, T)$ that

$$
\Theta_{\varepsilon}(\cdot, t), \Theta_{\varepsilon}^{ \pm}(\cdot, t) \rightarrow \theta(\cdot, t) \quad \text { strongly in } C^{0, \beta}(\bar{\Omega}) \quad \text { as } h \rightarrow 0 .
$$

Proof. On noting (3.1a, b) and (1.19), the bounds (2.74), (2.90) and (2.91) imply that

$$
\begin{gathered}
\left\|\underline{U}_{\varepsilon}^{+}\right\|_{L^{\infty}\left(0, T ; \underline{W}^{\left.1,2+\delta_{1}(\Omega)\right)}\right.}^{2}+\left\|\Theta_{\varepsilon}^{( \pm)}\right\|_{L^{\infty}\left(0, T ; H^{1}(\Omega)\right)}^{2}+\left\|\left[\Xi_{\varepsilon}\left(\Theta_{\varepsilon}^{-}\right)\right]^{\frac{1}{2}} \nabla W_{\varepsilon}^{+}\right\|_{L^{2}\left(0, T ; \underline{L}^{2}(\Omega)\right)}^{2} \\
(3.12)+\left\|\bar{\tau}^{\frac{1}{2}} \frac{\partial \Theta_{\varepsilon}}{\partial t}\right\|_{L^{2}\left(0, T ; H^{1}(\Omega)\right)}^{2}+\left\|\mathcal{G} \frac{\partial \Theta_{\varepsilon}}{\partial t}\right\|_{L^{2}\left(0, T ; H^{1}(\Omega)\right)}^{2}+\tau^{-\frac{1}{2}}\left\|\bar{\tau}^{\frac{1}{2}} \frac{\partial \Theta_{\varepsilon}}{\partial t}\right\|_{L^{2}\left(\Omega_{T}\right)}^{2} \leq C .
\end{gathered}
$$

Furthermore, we deduce from (3.2) and (3.12) that

$$
\left\|\Theta_{\varepsilon}-\Theta_{\varepsilon}^{ \pm}\right\|_{L^{2}\left(0, T ; H^{1}(\Omega)\right)}^{2} \leq\left\|\bar{\tau} \frac{\partial \Theta_{\varepsilon}}{\partial t}\right\|_{L^{2}\left(0, T ; H^{1}(\Omega)\right)}^{2} \leq C \tau .
$$

Hence on noting (3.12), (3.13), $\Theta_{\varepsilon}(\cdot, t) \in K^{h}, \underline{U}_{\varepsilon}^{+}(\cdot, t) \in \underline{\widehat{S}}^{h}$, and the compactness result [12, (1.12a)] we can choose a subsequence $\left\{\underline{U}_{\varepsilon}^{+}, \bar{\Theta}_{\varepsilon}, W_{\varepsilon}^{+}\right\}_{h}$ such that the convergence results (3.4), (3.5a-c) and (3.6a) hold. Then (3.4) and Theorem 2.1 
yield, on noting [12, (1.12b)], our assumption on $\Theta_{\varepsilon}^{0}$ and (2.7) that the subsequence satisfies the additional initial and integral conditions.

The desired results (3.6b c) follow from (2.66b), (2.5), (3.12), (2.65), (1.7) and (1.4); see [12, Lemma 3.1] for details.

For any $\underline{\eta} \in L^{2}\left(0, T ; \underline{H}^{2}(\Omega)\right)$, we choose $\underline{\chi} \equiv \pi^{h} \eta$ in (2.3a). The desired result (3.7) then follows from (2.6), a trace inequality, (3.12), (1.4), (3.6c), (3.5c) and a density result. We have from (3.3a) and (3.7) that

$$
\begin{gathered}
\int_{\Omega_{T}} c(\theta) \mathcal{C} \underline{\underline{\mathcal{E}}}\left(\underline{u}-\underline{U}_{\varepsilon}^{+}\right): \underline{\underline{\mathcal{E}}}\left(\underline{u}-\underline{U}_{\varepsilon}^{+}\right) \mathrm{d} x \mathrm{~d} t \\
(3.14)=\int_{\Omega_{T}}\left[c(\theta) \mathcal{C} \underline{\underline{\mathcal{E}}}\left(\underline{u}-\underline{U}_{\varepsilon}^{+}\right): \underline{\underline{\mathcal{E}}}(\underline{u})+\left[c(\theta)-c\left(\Theta_{\varepsilon}^{-}\right)\right] \mathcal{C} \underline{\underline{\mathcal{E}}}\left(\underline{U}_{\varepsilon}^{+}\right): \underline{\underline{\mathcal{E}}}\left(\underline{U}_{\varepsilon}^{+}\right)\right] \mathrm{d} x \mathrm{~d} t .
\end{gathered}
$$

The desired result (3.6d) then follows from (3.14), on noting (1.4), (1.19), (3.5c) and (3.6c).

It follows from (2.92), (2.89), (2.8), (3.1a) b) and our assumptions on $\left\{\tau_{n}\right\}_{n=1}^{N}$ and $\varepsilon$ that

$$
\left\|\Delta^{h} \Theta_{\varepsilon}^{( \pm)}\right\|_{L^{2}\left(\Omega_{T}\right)}^{2}+\left\|\underline{U}_{\varepsilon}^{+}\right\|_{L^{4}\left(0, T ; \underline{W}^{1,4}(\Omega)\right)}^{4} \leq C(T) .
$$

The desired results (3.9) and (3.10a,d) then follow from (3.15), (2.14), (2.7), (2.9), (3.12), (3.5a), elliptic regularity as $\Omega$ is convex polygonal, and (3.4); see [12, Lemma 3.1] for details. Furthermore, it follows from (3.10a) and (2.16a) that (3.10b) holds on extracting a further subsequence.

Finally, (3.10c) for $\Theta_{\varepsilon}$ follows from (3.10b), (3.5b), [12, (1.12a)] and the compact embedding $W^{1, s}(\Omega) \hookrightarrow C^{0, \beta}(\bar{\Omega})$, where $\beta<1-\frac{2}{s}$. To prove the result on $\Theta_{\varepsilon}^{ \pm}$in (3.10c), we note the following. For any $\beta \in(0,1)$ and any $\bar{s} \in\left(\frac{2}{1-\beta}, s\right)$ it follows on noting the above compact embedding, (3.13) and (3.10b) that

$$
\begin{aligned}
& \left\|\Theta_{\varepsilon}-\Theta_{\varepsilon}^{ \pm}\right\|_{L^{2}\left(0, T ; C^{0, \beta}(\bar{\Omega})\right)} \leq\left\|\Theta_{\varepsilon}-\Theta_{\varepsilon}^{ \pm}\right\|_{L^{2}\left(0, T ; W^{1, \bar{s}}(\Omega)\right)} \\
& \quad \leq\left\|\Theta_{\varepsilon}-\Theta_{\varepsilon}^{ \pm}\right\|_{L^{2}\left(0, T ; H^{1}(\Omega)\right)}^{\alpha}\left\|\Theta_{\varepsilon}-\Theta_{\varepsilon}^{ \pm}\right\|_{L^{2}\left(0, T ; W^{1, s}(\Omega)\right)}^{1-\alpha} \leq C \tau^{\frac{\alpha}{2}}
\end{aligned}
$$

where $\alpha=\frac{2(s-\bar{s})}{(s-2) \bar{s}} \in(0,1)$. Combining (3.16) $), \tau \rightarrow 0$ and the established result on $\Theta_{\varepsilon}$ in (3.10c) yields the desired result (3.10c) for $\Theta_{\varepsilon}^{ \pm}$. The final result (3.11) follows immediately from (3.10c).

Remark 3.1. The condition $\theta^{0} \in H^{2}(\Omega)$ with $\frac{\partial \theta^{0}}{\partial \nu}=0$ can be relaxed, but it is not particularly restrictive; see, e.g., [11.

From (3.12), (2.66a), (2.65), (1.7) and (3.11) we see that we can only control $\nabla W_{\varepsilon}^{+}$on the set where $\Xi_{\varepsilon}\left(\Theta_{\varepsilon}^{-}\right)$is bounded below independently of $\varepsilon$, and hence $h$, as $\varepsilon \rightarrow 0$ and $h \rightarrow 0$, i.e., on the set where $|\theta|<1$. Therefore in order to construct the appropriate limits as $h \rightarrow 0$, we introduce the following open subsets of $\bar{\Omega}$. For any $\rho \in(0,1)$, we define for a.a. $t \in(0, T)$,

$$
B_{\rho}(t):=\{x \in \bar{\Omega}:|\theta(x, t)|<1-\rho\} .
$$

We have from (3.11) (see [12] for details) that for a.a. $t \in(0, T)$ and any $\rho \in\left(0, \rho_{0}\right)$, there exists an $h_{0}(\rho, t)$ such that for all $h \leq h_{0}(\rho, t)$ there exist collections of simplices $\mathcal{T}_{B, \rho}^{h}(t) \subset \mathcal{T}^{h}$ such that

$$
B_{\rho}(t) \subset B_{\rho}^{h}(t):=\bigcup_{\sigma \in \mathcal{T}_{B, \rho}^{h}(t)} \bar{\sigma} \subset B_{\frac{\rho}{2}}(t) .
$$


In addition for a.a. $t \in(0, T)$ and any fixed $\rho \in\left(0, \widehat{\rho}_{0}\right)$, where $\widehat{\rho}_{0}:=\min \left\{\rho_{0}, \frac{1}{2}\right\}$, it follows from (3.17), (3.11) and our assumption on $\varepsilon$ in Lemma 3.1 that there exists an $\widehat{h}_{0}(\rho, t) \leq h_{0}(\rho, t)$ such that for $h \leq \widehat{h}_{0}(\rho, t)$,

$1-2 \rho \leq\left|\Theta_{\varepsilon}^{ \pm}(x, t)\right| \quad \forall x \notin B_{\rho}(t), \quad\left|\Theta_{\varepsilon}^{ \pm}(x, t)\right| \leq 1-\frac{\rho}{2} \quad \forall x \in B_{\rho}(t) \quad$ and $\quad \varepsilon \leq \rho$.

Lemma 3.2. Let all the assumptions of Lemma 3.1 hold. Then for a.a. $t \in(0, T)$ there exists a function

$w(\cdot, t) \equiv-\gamma \Delta \theta(\cdot, t)-\gamma^{-1} \theta(\cdot, t)+\frac{1}{2}\left[c^{\prime}(\theta) \mathcal{C} \underline{\underline{\mathcal{E}}}(\underline{u}): \underline{\underline{\mathcal{E}}}(\underline{u})\right](\cdot, t) \in H_{l o c}^{1}(\{|\theta(\cdot, t)|<1\})$, where $\{|\theta(\cdot, t)|<1\}:=\{x \in \bar{\Omega}:|\theta(x, t)|<1\}$. Moreover, on extracting a further subsequence from the subsequence $\left\{\underline{U}_{\varepsilon}^{+}, \Theta_{\varepsilon}, W_{\varepsilon}^{+}\right\}_{h}$ in Lemma 3.1, it follows as $h \rightarrow$ 0 that

$$
\Xi_{\varepsilon}\left(\Theta_{\varepsilon}^{-}\right) \nabla W_{\varepsilon}^{+} \rightarrow \mathcal{H}_{\{|\theta|<1\}} b(\theta) \nabla w \quad \text { weakly in } L^{2}\left(0, T ; \underline{L}^{2}(\Omega)\right),
$$

where $\mathcal{H}_{\{|\theta|<1\}}$ is the characteristic function of the set $\{|\theta|<1\}:=\left\{(x, t) \in \Omega_{T}\right.$ : $|\theta(x, t)|<1\}$.

Proof. It follows from (3.12) and (2.66a) that

$$
\left\|\Xi_{\varepsilon}\left(\Theta_{\varepsilon}^{-}\right) \nabla W_{\varepsilon}^{+}\right\|_{L^{2}\left(0, T ; L^{2}(\Omega)\right)}^{2} \leq C .
$$

Hence (3.22) implies that there exists a function $\underline{z} \in L^{2}\left(0, T ; \underline{L}^{2}(\Omega)\right)$, and on extracting a further subsequence from the subsequence $\left\{\underline{U}_{\varepsilon}^{+}, \Theta_{\varepsilon}, W_{\varepsilon}^{+}\right\}_{h}$ in Lemma 3.1 . it follows as $h \rightarrow 0$ that

$$
\Xi_{\varepsilon}\left(\Theta_{\varepsilon}^{-}\right) \nabla W_{\varepsilon}^{+} \rightarrow \underline{z} \quad \text { weakly in } L^{2}\left(0, T ; \underline{L}^{2}(\Omega)\right) .
$$

We now identify $\underline{z}$.

First, we consider a fixed $\rho \in\left(0, \rho_{0}\right)$. It follows from (1.7), (2.65), (2.66a), (3.19) and (3.12) that for a.a. $t \in(0, T)$ and for all $h \leq \widehat{h}_{0}(\rho, t)$,

$$
\begin{aligned}
& \rho\left(1-\frac{\rho}{4}\right)\left|\nabla W_{\varepsilon}^{+}(\cdot, t)\right|_{0, B_{\rho}(t)}^{2}=b\left(1-\frac{\rho}{2}\right)\left|\nabla W_{\varepsilon}^{+}(\cdot, t)\right|_{0, B_{\rho}(t)}^{2} \\
& \quad \leq b_{\varepsilon}\left(1-\frac{\rho}{2}\right)\left|\nabla W_{\varepsilon}^{+}(\cdot, t)\right|_{0, B_{\rho}(t)}^{2} \leq\left|\left(\left[\Xi_{\varepsilon}\left(\Theta_{\varepsilon}^{-}\right)\right]^{\frac{1}{2}} \nabla W_{\varepsilon}^{+}\right)(\cdot, t)\right|_{0}^{2} \leq C(t) .
\end{aligned}
$$

From (3.24), (3.18), (2.66a) and (3.19) we have for a.a. $t \in(0, T)$ and for all $h \leq \widehat{h}_{0}(\rho, t)$,

$$
\begin{gathered}
\left|\left(\Xi_{\varepsilon}\left(\Theta_{\varepsilon}^{-}\right) \nabla W_{\varepsilon}^{+}\right)(\cdot, t)\right|_{0, \Omega \backslash B_{\rho}(t)}^{2} \leq \max _{x \in \Omega \backslash B_{2 \rho}(t)} b_{\varepsilon}\left(\Theta_{\varepsilon}^{-}(x)\right)\left|\left(\left[\Xi_{\varepsilon}\left(\Theta_{\varepsilon}^{-}\right)\right]^{\frac{1}{2}} \nabla W_{\varepsilon}^{+}\right)(\cdot, t)\right|_{0, \Omega \backslash B_{\rho}(t)}^{2} \\
(3.25) \quad \leq C(t) b_{\varepsilon}(1-4 \rho) \leq C(t) \rho .
\end{gathered}
$$

On noting (3.15) we have for a.a. $t \in(0, T)$ that

$$
\left|\Delta^{h} \Theta_{\varepsilon}^{+}(\cdot, t)\right|_{0}^{2}+\left\|\underline{U}_{\varepsilon}^{+}(\cdot, t)\right\|_{1,4}^{4} \leq C(t) .
$$

It follows from (3.26) and (3.6d), on extracting a further subsequence, that for a.a. $t \in(0, T)$ and as $h \rightarrow 0$,

$$
\begin{array}{rlrl}
\Delta^{h} \Theta_{\varepsilon}^{+}(\cdot, t) & \rightarrow \Delta \theta(\cdot, t) & & \text { weakly in } L^{2}(\Omega), \\
\underline{U}_{\varepsilon}^{+}(\cdot, t) & \rightarrow \underline{u}(\cdot, t) & \text { weakly in } \underline{W}^{1,4}(\Omega) \text { and strongly in } \underline{H}^{1}(\Omega) ;
\end{array}
$$


see [12, as for (3.10a), for details of the former. Combining (2.71), (2.14), (3.1b), (3.19) and (3.18) yields for a.a. $t \in(0, T)$ and for all $h \leq \widehat{h}_{0}\left(\frac{\rho}{4}, t\right)$ that on $B_{\rho}(t)$, (3.28)

$$
W_{\varepsilon}^{+}(\cdot, t) \equiv-\gamma \Delta^{h} \Theta_{\varepsilon}^{+}(\cdot, t)-\gamma^{-1} \Theta_{\varepsilon}^{-}(\cdot, t)+\frac{1}{2}\left(Q^{h}\left[c^{\prime}\left(\Theta_{\varepsilon}^{-}\right) \mathcal{C} \underline{\underline{\mathcal{E}}}\left(\underline{U}_{\varepsilon}^{+}\right): \underline{\underline{\mathcal{E}}}\left(\underline{U}_{\varepsilon}^{+}\right)\right]\right)(\cdot, t) .
$$

If $v_{i}^{(h)} \in L^{4}(\Omega), i=1,2$, then for any $\eta \in H^{2}(\Omega)$ we have that

$$
\left(Q^{h}\left[v_{1}^{h} v_{2}^{h}\right]-v_{1} v_{2}, \eta\right)=\left(v_{1}^{h} v_{2}^{h}-v_{1} v_{2}, \eta\right)+\left(\left(Q^{h}-I\right)\left[v_{1}^{h} v_{2}^{h}\right],\left(I-\pi^{h}\right) \eta\right)
$$

$$
+\left[\left(Q^{h}\left[v_{1}^{h} v_{2}^{h}\right], \pi^{h} \eta\right)-\left(Q^{h}\left[v_{1}^{h} v_{2}^{h}\right], \pi^{h} \eta\right)^{h}\right] .
$$

It then follows from (3.29), (2.6), (2.9) and a density argument that for $i=1,2$,

$$
\begin{array}{rlrl}
v_{i}^{h} & \rightarrow v_{i} & \text { strongly in } L^{2}(\Omega) \text { and weakly in } L^{4}(\Omega), \\
\Longrightarrow Q^{h}\left[v_{1}^{h} v_{2}^{h}\right] & \rightarrow v_{1} v_{2} \quad \text { weakly in } L^{2}(\Omega) .
\end{array}
$$

We then have from (3.28), (3.27a, b), (3.11), (1.4) and (3.30) for a.a.t $t \in(0, T)$ that as $h \rightarrow 0$,

$W_{\varepsilon}^{+}(\cdot, t) \rightarrow-\gamma \Delta \theta(\cdot, t)-\gamma^{-1} \theta(\cdot, t)+\frac{\gamma}{2}\left[c^{\prime}(\theta) \mathcal{C} \underline{\underline{\mathcal{E}}}(\underline{u}): \underline{\underline{\mathcal{E}}}(\underline{u})\right](\cdot, t)$ weakly in $L^{2}\left(B_{\rho}(t)\right)$.

This together with (3.24) yields

$$
W_{\varepsilon}^{+}(\cdot, t) \rightarrow w(\cdot, t) \quad \text { weakly in } H^{1}\left(B_{\rho}(t)\right) .
$$

Combining (3.23), (3.31) and (3.6b) yields for a.a. $t \in(0, T)$ that as $h \rightarrow 0$,

$$
\left[\Xi_{\varepsilon}\left(\Theta_{\varepsilon}^{-}\right) \nabla W_{\varepsilon}^{+}\right](\cdot, t) \rightarrow b(\theta(\cdot, t)) \nabla w(\cdot, t) \quad \text { weakly in } \underline{L}^{2}\left(B_{\rho}(t)\right) .
$$

Repeating (3.24) - (3.32) for all $\rho \in\left(0, \widehat{\rho}_{0}\right)$ yields (3.20) and, on noting (3.25) and (3.23), the desired result (3.21).

Theorem 3.1. Let the assumptions of Lemma 3.2 hold. Then there exists a subsequence of $\left\{\underline{U}_{\varepsilon}^{+}, \Theta_{\varepsilon}, W_{\varepsilon}^{+}\right\}_{h}$, where $\left\{\underline{U}_{\varepsilon}^{+}, \Theta_{\varepsilon}, W_{\varepsilon}^{+}\right\}$solve $\left(\mathrm{P}_{\varepsilon}^{h, \tau}\right)$, and functions $\{\underline{u}, \theta, w\}$ satisfying (3.4), (3.9), and (3.20). In addition, as $h \rightarrow 0$ the following hold: (3.5a-c), (3.6a-d), (3.10a-d), (3.11) for a.a. $t \in(0, T)$, and (3.21). Furthermore, we have that $\{\underline{u}, \theta, w\}$ fulfill $\theta(\cdot, 0)=\theta^{0}(\cdot)$ in $L^{2}(\Omega)$ and satisfy (3.7), (3.20) and

$$
\gamma \int_{0}^{T}\left\langle\frac{\partial \theta}{\partial t}, \eta\right\rangle \mathrm{d} t+\int_{\{|\theta|<1\}} b(\theta) \nabla w \cdot \nabla \eta \mathrm{d} x \mathrm{~d} t=0 \quad \forall \eta \in L^{2}\left(0, T ; H^{1}(\Omega)\right) .
$$

Proof. We need to prove only (3.33). For any $\eta \in H^{1}\left(0, T ; H^{2}(\Omega)\right)$ we choose $\chi \equiv \pi^{h} \eta$ in (3.3b). The desired result (3.33) then follows from (2.9), the embedding $H^{1}(0, T ; X) \hookrightarrow C([0, T] ; X),(3.12),(2.6),(2.10)$, (3.5b), (2.66a), (3.21) and the denseness of $H^{1}\left(0, T ; H^{2}(\Omega)\right)$ in $L^{2}\left(0, T ; H^{1}(\Omega)\right)$; see [12, Theorem 3.6] for details.

\section{Numerical Results}

Before presenting some numerical results, we briefly state algorithms for solving the resulting system of algebraic equations for $\left\{\underline{U}_{\varepsilon}^{n}, \Theta_{\varepsilon}^{n}, W_{\varepsilon}^{n}\right\}$ arising at each time level from the approximation $\left(\mathrm{P}_{\varepsilon}^{h, \tau}\right)$. As (2.3a) is independent of $\left\{\Theta_{\varepsilon}^{n}, W_{\varepsilon}^{n}\right\}$, we first solve the resulting linear equation to obtain $\underline{U}_{\varepsilon}^{n}$. To this end we employ a preconditioned conjugate gradient solver. Then the nonlinear equations $(2.3 \mathrm{~b}-\mathrm{c})$ are solved, using the same "Gauss-Seidel type" iteration as in [12, §4]. 
In order to define the initial shape of the void we introduce the following function. Given $z \in \mathbb{R}^{2}$ and $R \in \mathbb{R}_{>0}$ we define

$$
v(z, R ; x):= \begin{cases}-1 & r(x) \leq R-\frac{\gamma \pi}{2} \\ \sin \left(\frac{r(x)-R}{\gamma}\right) & |r(x)-R|<\frac{\gamma \pi}{2}, \quad \text { where } r(x):=|x-z| . \\ 1 & r(x) \geq R+\frac{\gamma \pi}{2}\end{cases}
$$

(4.1) represents a circular void with radius $R$. In line with the asymptotics of the phase field approach (see \$1), the interfacial thickness of $v$ is equal to $\gamma \pi$. For the initial data to $(\mathrm{P})$ we chose $\theta^{0}$ to be either (i) one circle or (ii) two circles; that is,

$$
\theta^{0}(x)=v(z, R ; x) \quad \text { or } \quad(i i) \quad \theta^{0}(x)=\max \{v(z, R ; x)+v(\tilde{z}, \widetilde{R} ; x)-1,-1\} .
$$

We note that in the absence of elastic stresses both these choices of $\theta^{0}$ are close to being steady states of $(\mathrm{P})$, provided that in $(i i)$ the two circular voids are sufficiently far apart, e.g., $|z-\tilde{z}|>R+\widetilde{R}+2 \gamma \pi$.

Throughout the given domain, $\Omega=(-L, L) \times(-L, L)$ is partitioned into rightangled isosceles triangles such that there are approximately 8 mesh points across the interface. On using the adaptive finite element code ALBERT 1.0 (see 43]), we implemented the same mesh refinement strategy as in [12. In particular, to improve efficiency we use a modified approximation $\left(\widetilde{\mathrm{P}}_{\varepsilon}^{h, \tau}\right)$; see [12, Remark 2.10], and set $\Theta_{\varepsilon}^{0} \equiv \pi^{h} \theta^{0}$. Now we have to solve for $\left\{\Theta_{\varepsilon}^{n}, W_{\varepsilon}^{n}\right\}$ only in the interfacial region, $\left|\Theta_{\varepsilon}^{n-1}\right|<1$. Hence we use a refined mesh with mesh size $h_{f}=\frac{2^{\frac{3}{2}} L}{N_{f}}$ in this interfacial region, and a coarser mesh of mesh size $h_{c}=\frac{2^{\frac{3}{2}} L}{N_{c}}$ away from the interface. Here $N_{f}$ and $N_{c}$ are parameters; see [12, §5]. Furthermore, we choose $N_{f}$ such that there are always at least approximately 8 mesh points across the interface in each direction, i.e., $h_{f} \leq \frac{3 \sqrt{2}}{32} \gamma \pi$.

Throughout this section, we restrict ourselves to isotropic elasticity. Hence the assumption (2.23) is satisfied and all our theoretical results in this paper apply. If $\mathcal{C}$ is isotropic, (1.11), then it can be described by its nonzero elements

$$
\mathcal{C}_{1111}=\mathcal{C}_{2222}=2 \mu+\lambda, \mathcal{C}_{1122}=\lambda, \mathcal{C}_{1212}=\mu,
$$

where $\mu \in \mathbb{R}_{>0}$ and $\lambda \in \mathbb{R}_{\geq 0}$ are the Lamé moduli.

The following computations are inspired by the results in [14, Figures 9 and 10]. It was noticed there that the void evolution depends strongly on the dimensionless parameter $\Lambda=\frac{S_{\infty}^{2} R}{\beta \varsigma}$, where $S_{\infty}$ is the maximal stress applied externally, $\beta=\frac{\mu(2 \mu+3 \lambda)}{\mu+\lambda}, R$ is the initial radius of the void, as in (4.1), and $\varsigma$ is surface energy density, which without loss of generality is taken as $\frac{\pi}{2}$ throughout this paper. Unfortunately, the authors did not provide their exact dimensions, but it seems that there $L \approx 4 R$ and $R \approx 7 \gamma$. Throughout our experiments we set $\Omega$ to be the unit square, $L=0.5$; hence these values correspond to $R \approx \frac{1}{8}$ and $\gamma \approx \frac{1}{56} \approx \frac{1}{18 \pi}$. In what follows we set $R=\frac{1}{8}, \underline{\underline{S}}=\left(\begin{array}{ll}0 & 0 \\ 0 & 1\end{array}\right)$ for the pure traction boundary condition (1.13) $\Rightarrow S_{\infty}=1$, and choose $\gamma \in\left\{\frac{1}{12 \pi}, \frac{1}{24 \pi}, \frac{1}{48 \pi}\right\}$. Finally, let $\mathcal{C}$ be defined by (4.3) with $\lambda=\mu=\frac{1}{10 \Lambda \pi}$, where $\Lambda \in\left\{\frac{1}{8}, \frac{1}{5}, \frac{1}{2}\right\}$.

First, we conducted the following convergence experiments. Setting $c_{0}(\gamma)=\gamma^{2}$ in (1.4), we repeated the same experiment with decreasing values of $\gamma$, i.e., $\gamma=$ $\frac{1}{12 \pi}, \frac{1}{24 \pi}, \frac{1}{48 \pi}$. In particular, we set $\Lambda=\frac{1}{8}, T=0.02, \tau_{n}=\tau=(\gamma 24 \pi)^{2} \times 10^{-6}$, 


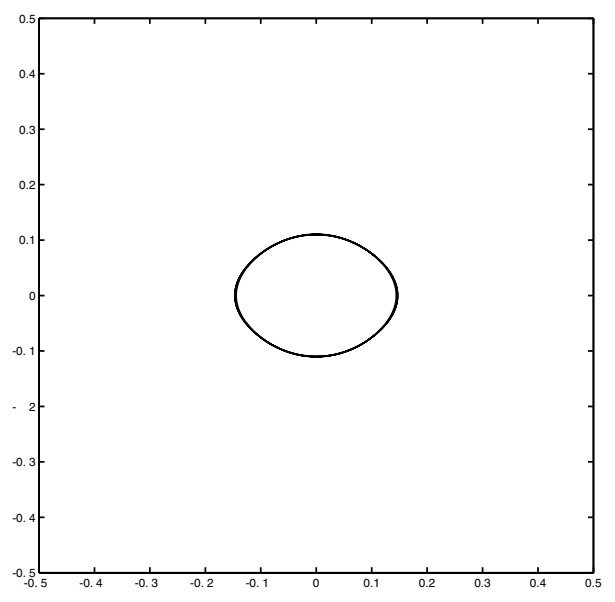

Figure 1. $\left(\underline{\underline{S}}=\left(\begin{array}{ll}0 & 0 \\ 0 & 1\end{array}\right), \Lambda=\frac{1}{8}\right)$ Comparison of zero level sets for $\Theta_{\varepsilon}(x, t)$ at time $T=0.02$ for $\gamma=\frac{1}{24 \pi}$ and $\gamma=\frac{1}{48 \pi}$.

$\varepsilon=\gamma 24 \pi \times 10^{-5}$ and used the appropriate refinement parameters $N_{f}=\frac{32}{3} \frac{1}{\gamma \pi}$, $N_{c}=\frac{N_{f}}{8}$. The steady state solutions for this setup agreed very well for the different values of $\gamma$. Hence we are satisfied that the converged solution is very close to the sharp interface limit. See Figure 1, where we superimpose the steady states for $\gamma=\frac{1}{24 \pi}$ and $\gamma=\frac{1}{48 \pi}$.

For the remaining experiments, we fix $\varepsilon=10^{-5}$ and set $c_{0}=10^{-3}$ in (1.4). In our first run, we chose $\Lambda=\frac{1}{5}$ as in [14, Fig. 9]. This yields $\lambda=\mu=\frac{1}{2 \pi}$. The other parameters were chosen as follows: $\gamma=\frac{1}{12 \pi}, T=0.02, \tau_{n}=\tau=$ $1.5 \times 10^{-5}$. As initial data we chose (4.2) $(i)$ with $z=(0,0), R=\frac{1}{8}$. The refinement parameters were $N_{f}=128$ and $N_{c}=16$. In Figure 2 we plot the zero level sets for $\Theta_{\varepsilon}(x, t)$ at different times. Note that the last plot is a numerical steady state. Furthermore, the figure contains plots of the principal elastic stress field and the elastic energy density at time $t=T$. Here the former is defined as $\max \{|\alpha|$ : $\alpha$ is an eigenvalue of $\left.c\left(\Theta_{\varepsilon}^{-}\right) \mathcal{C} \underline{\underline{\mathcal{E}}}\left(\underline{U}_{\varepsilon}^{+}\right)\right\}$, whereas the elastic energy density is defined as $c\left(\Theta_{\varepsilon}^{-}\right) \mathcal{C} \underline{\underline{\mathcal{E}}}\left(\underline{U}_{\varepsilon}^{+}\right): \underline{\underline{\mathcal{E}}}\left(\underline{U}_{\varepsilon}^{+}\right)$. To simplify matters, both functions were evaluated at the vertices of the triangulation, where we used an arithmetic average of the functions' value on all adjacent triangles. One clearly notices that material is transported away from regions with high elastic energy density.

To check convergence, we repeated the same experiment with finer discretization parameters $\tau_{n}=\tau=5 \times 10^{-6}, N_{f}=256, N_{c}=32$ and the results were graphically indistinguishable from those in Figure 2 ,

For a smaller interfacial parameter $\gamma=\frac{1}{24 \pi}$ we observe a strikingly different behaviour; see Figures 3(a) and 3(b). The elliptic shape is no longer stable, and this leads to the development of a long slit. Here we see that the condition (3.8) need not always be satisfied in practice. Hence our convergence results for $\left(\mathrm{P}_{\varepsilon}^{h, \tau}\right)$ and a fixed $\gamma$ would only hold true until the void reaches the boundary of the domain and the material is separated into two parts. The evolution in this example indicates that the elastic stresses and the curvature would become singular in the sharp interface limit. Hence the sharp interface asymptotics, which assumes a bounded 

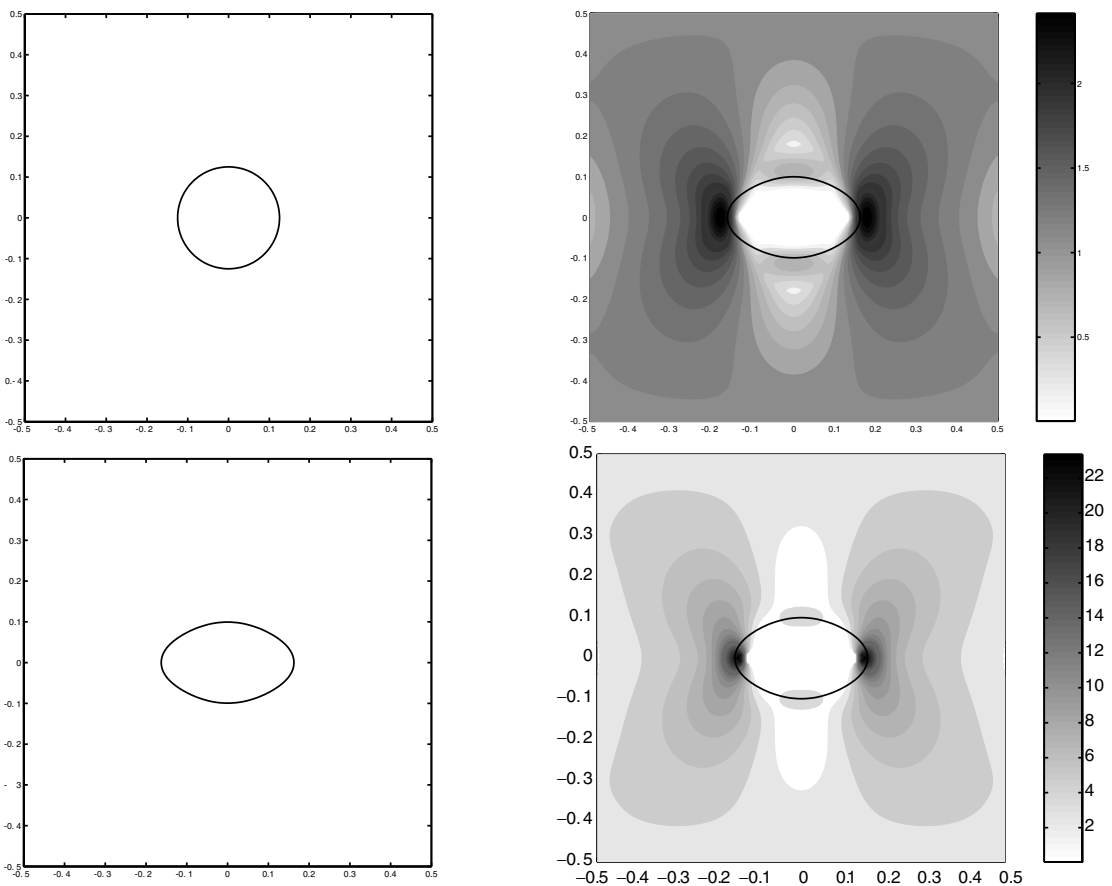

Figure 2. $\left(\underline{\underline{S}}=\left(\begin{array}{ll}0 & 0 \\ 0 & 1\end{array}\right), \Lambda=\frac{1}{5}, \gamma=\frac{1}{12 \pi}\right) \Theta_{\varepsilon}(x, t)$ at times $t=$ $0,0.02$ and elastic stress field and elastic energy density at time $t=0.02$.

curvature, breaks down. These singularities are related to the Asaro-Tiller-Grinfeld instability; see, e.g., 2], 32] and also [45]. Moreover, it is argued in [38] that a phase field model can be interpreted as a regularization of the singularities resulting from these instabilities. In fact there it is claimed that a phase field model might even be more realistic, since it is not clear that the sharp interface model is still plausible in situations where it leads to finite time singularities. We note that our results are in contrast to [14, Fig. 9], where the authors used a larger interfacial parameter $\gamma$. The discretization parameters used for our computation are $\tau_{n}=\tau=2.5 \times 10^{-6}$ and $N_{f}=256, N_{c}=32$.

The next run is for $\Lambda=\frac{1}{2}$ as in [14, Fig.10], i.e., $\lambda=\mu=\frac{1}{5 \pi}$. A computation for $\gamma=\frac{1}{12 \pi}, T=4 \times 10^{-5}, \tau_{n}=\tau=5 \times 10^{-7}$ and refinement parameters $N_{f}=128$, $N_{c}=16$ can be seen in Figure 4

Again we can observe a slightly different evolution for a smaller value of $\gamma$; see Figure 5. In particular, the developing cusps appear sharper and less smoothed out. One can again clearly see that material is transported away from regions with high elastic energy density. The parameters for this computation were $\gamma=\frac{1}{24 \pi}$, $T=10^{-5}, \tau_{n}=\tau=10^{-7}, N_{f}=256$ and $N_{c}=32$.

A run with parameters as in Figure 2 but $\underline{\underline{S}}=\left(\begin{array}{ll}0 & 1 \\ 1 & 0\end{array}\right)$ can be seen in Figure 6 , where the last plot is a numerical steady state.

If we choose a smaller interfacial parameter $\gamma=\frac{1}{24 \pi}$, the elastic effect tends to be more pronounced and the steady state shape is slightly different (see Figure 7), where we used the same discretization parameters as in Figure 3. 

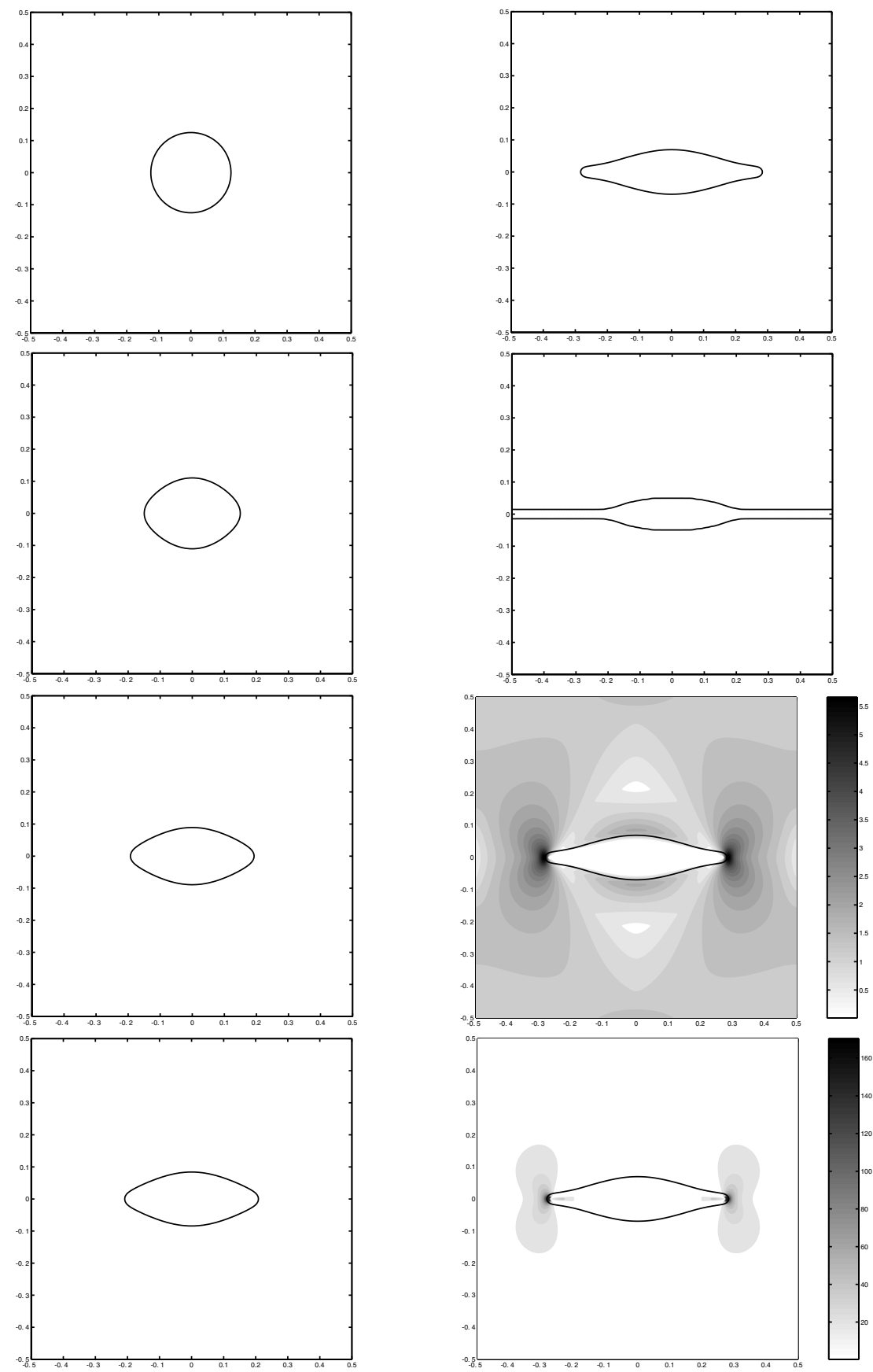

Figure 3. $\left(\underline{\underline{S}}=\left(\begin{array}{ll}0 & 0 \\ 0 & 1\end{array}\right), \Lambda=\frac{1}{5}, \gamma=\frac{1}{24 \pi}\right) \Theta_{\varepsilon}(x, t)$ at $t=0,1.5 \times$ $10^{-4}, 1.5 \times 10^{-3}, 3 \times 10^{-3}, 3.75 \times 10^{-3}, 5.25 \times 10^{-3}$ and elastic stress field and elastic energy density at time $t=3.75 \times 10^{-3}$. 

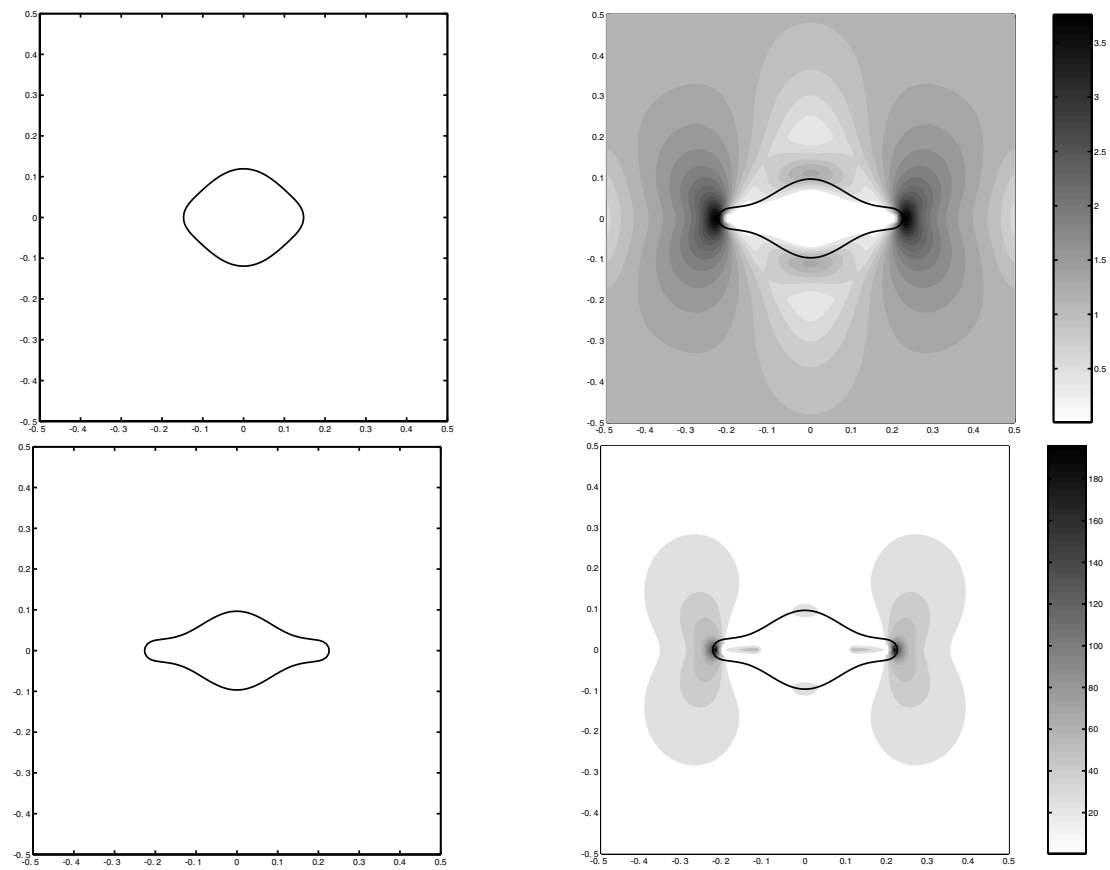

Figure 4. $\left(\underline{\underline{S}}=\left(\begin{array}{ll}0 & 0 \\ 0 & 1\end{array}\right), \Lambda=\frac{1}{2}, \gamma=\frac{1}{12 \pi}\right) \Theta_{\varepsilon}(x, t)$ at $t=10^{-5}, 4 \times 10^{-5}$ and elastic stress field and elastic energy density at time $t=4 \times 10^{-5}$.
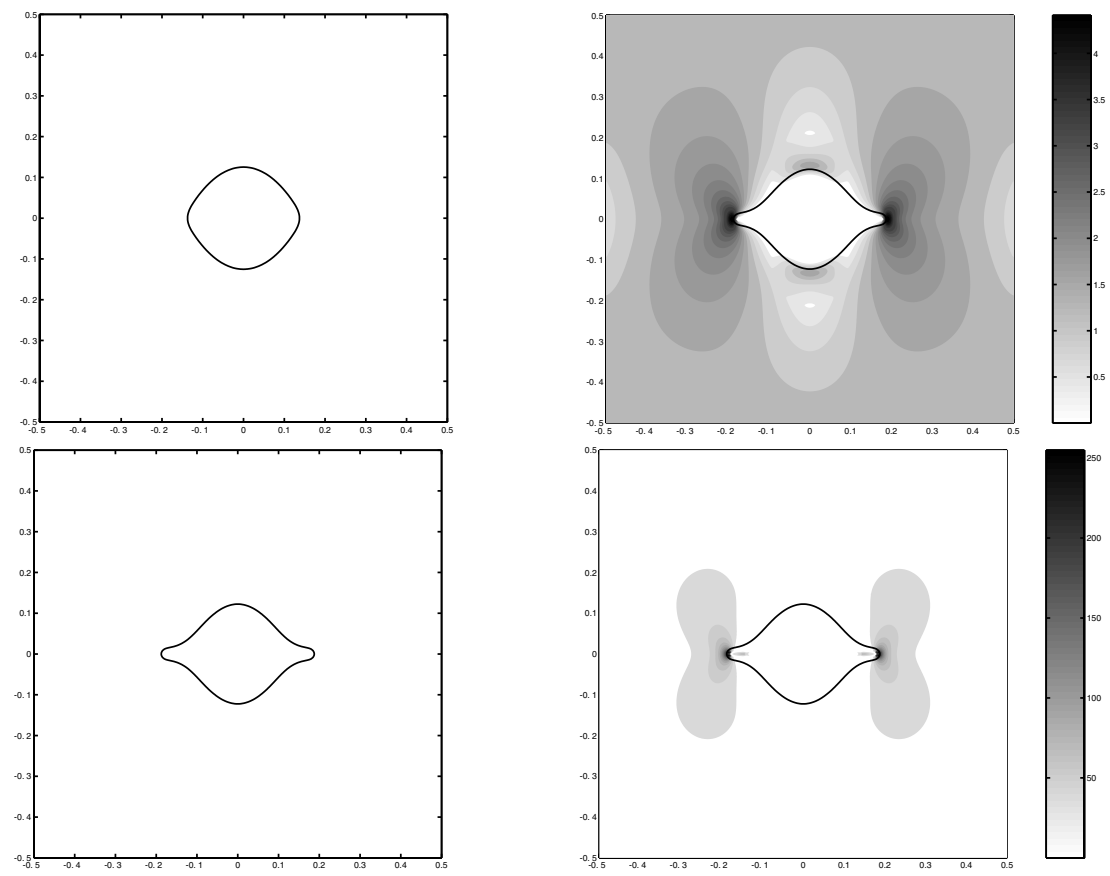

Figure 5. $\left(\underline{\underline{S}}=\left(\begin{array}{ll}0 & 0 \\ 0 & 1\end{array}\right), \Lambda=\frac{1}{2}, \gamma=\frac{1}{24 \pi}\right) \Theta_{\varepsilon}(x, t)$ at $t=3 \times 10^{-6}, 10^{-5}$ and elastic stress field and elastic energy density at $t=10^{-5}$. 

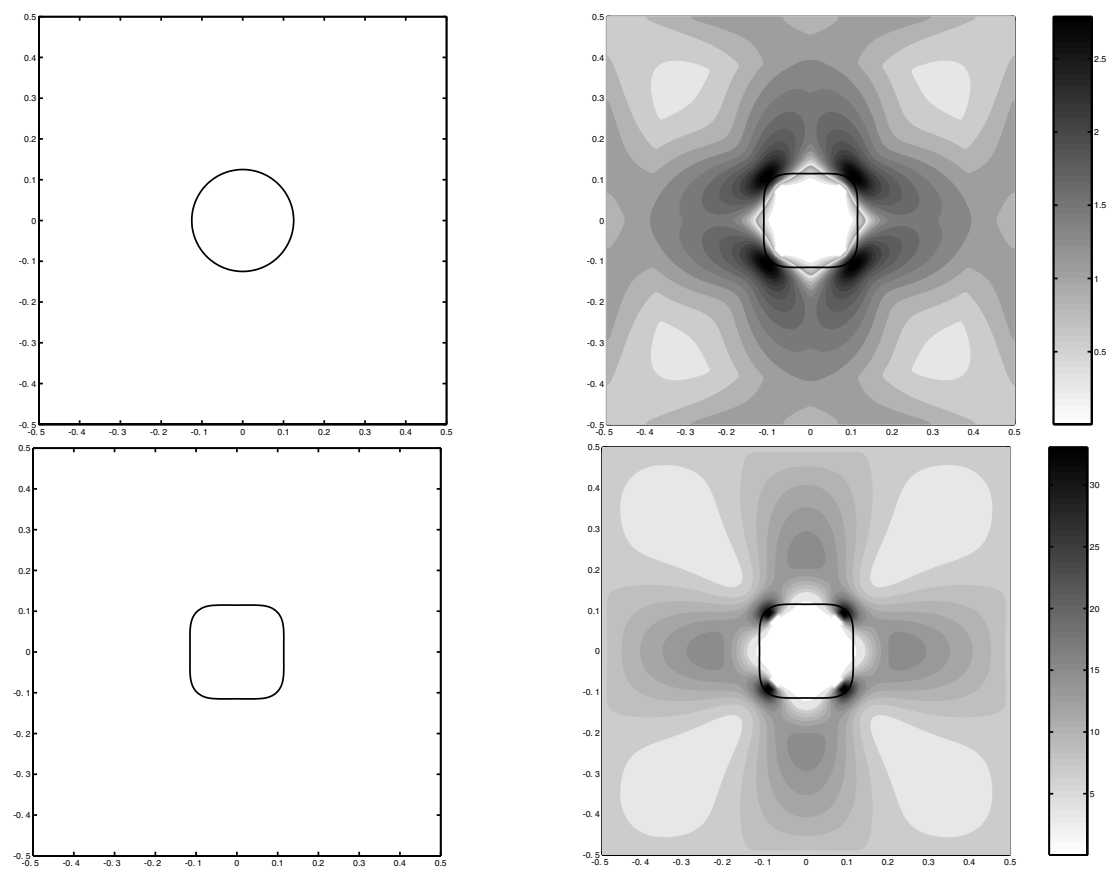

Figure $6 .\left(\underline{\underline{S}}=\left(\begin{array}{ll}0 & 1 \\ 1 & 0\end{array}\right), \Lambda=\frac{1}{5}, \gamma=\frac{1}{12 \pi}\right) \Theta_{\varepsilon}(x, t)$ at $t=0,0.02$ and elastic stress field and elastic energy density at time $t=0.02$.
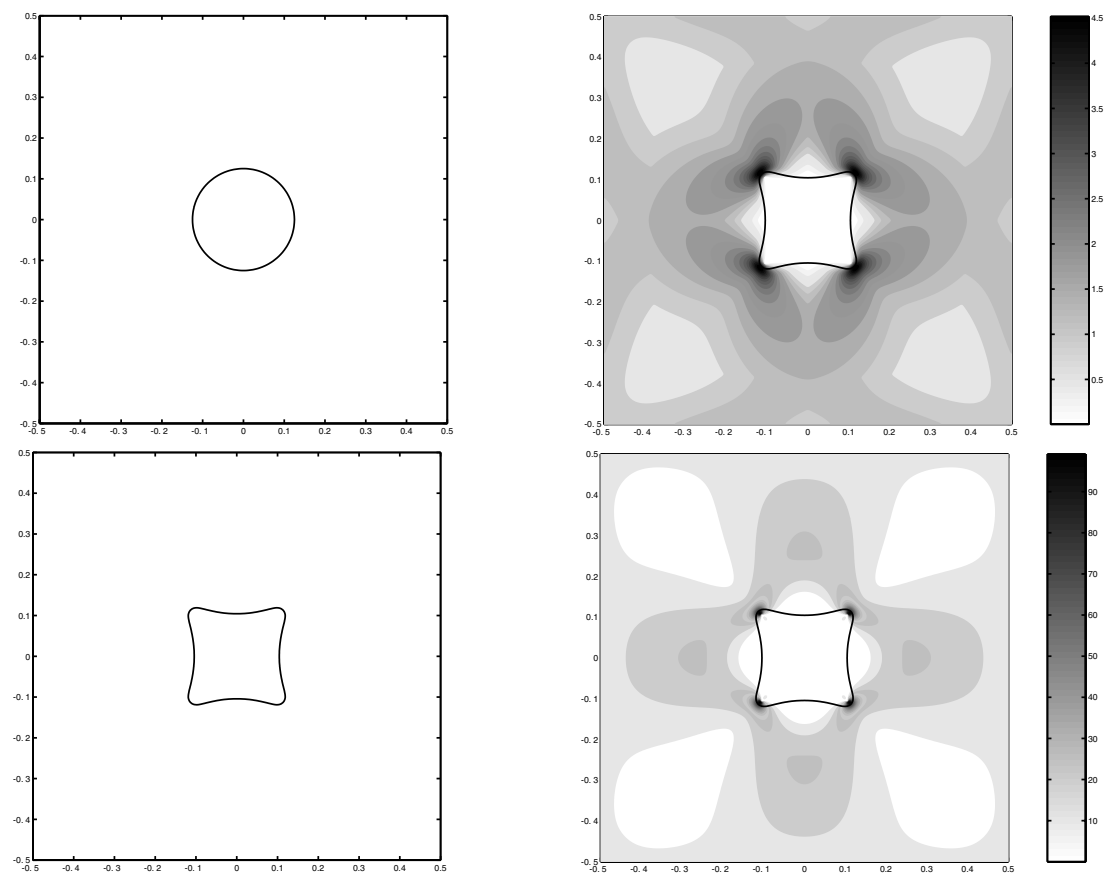

Figure 7. $\left(\underline{\underline{S}}=\left(\begin{array}{ll}0 & 1 \\ 1 & 0\end{array}\right), \Lambda=\frac{1}{5}, \gamma=\frac{1}{24 \pi}\right) \Theta_{\varepsilon}(x, t)$ at $t=0,0.02$ and elastic stress field and elastic energy density at time $t=0.02$. 

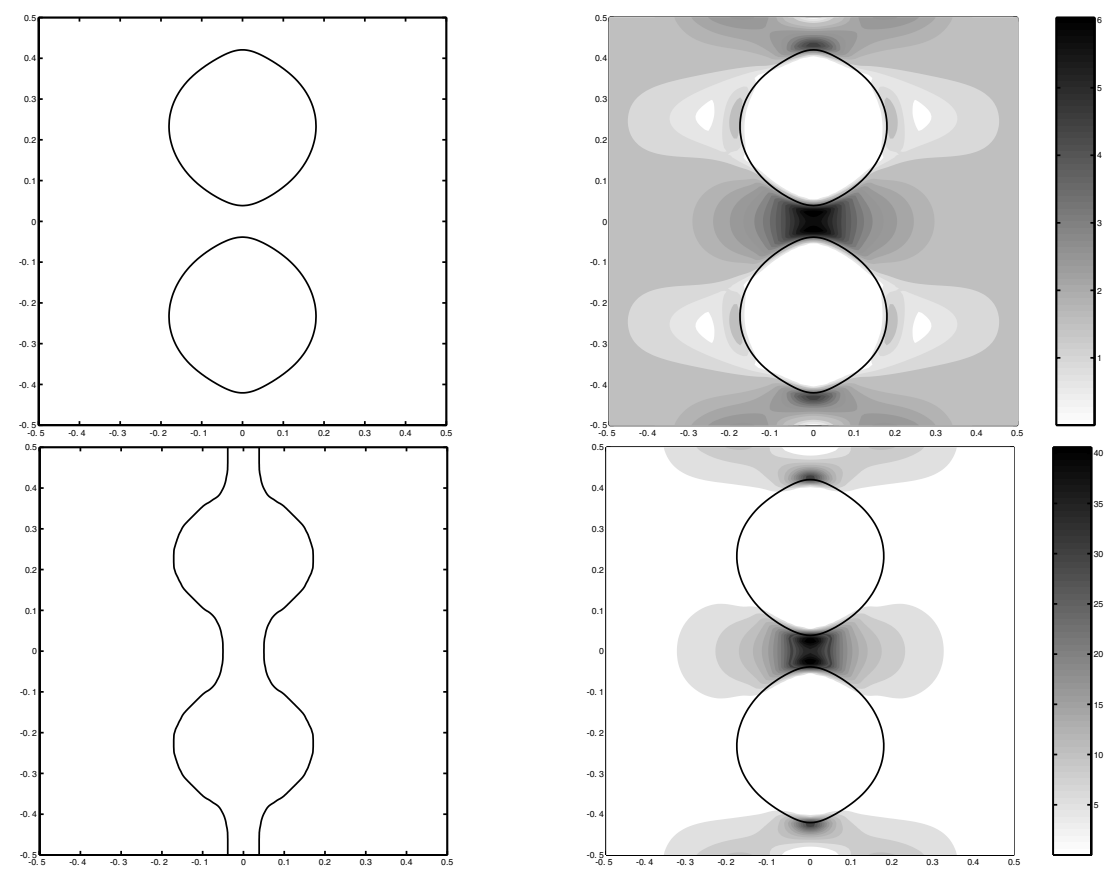

Figure 8. $\left(\underline{\underline{S}}=\left(\begin{array}{ll}1 & 0 \\ 0 & 0\end{array}\right), \mu=\frac{1}{2}, \lambda=0\right) \Theta_{\varepsilon}(x, t)$ at $t=10^{-5}, 5 \times$ $10^{-5}$ and elastic stress field and elastic energy density at time $t=10^{-5}$.

The last plot is a numerical steady state, and it is noteworthy that the steady state is nonconvex in contrast to Wulff shapes, which are minimizers of an anisotropic surface energy under a volume constraint.

For our last example, we chose $\mathcal{C}$ such that $\mathcal{C} \underline{\underline{\mathcal{E}}}(\underline{u})=\underline{\underline{\mathcal{E}}}(\underline{u})$, i.e., $\mu=\frac{1}{2}$ and $\lambda=0$, and set $\underline{\underline{S}}=\left(\begin{array}{ll}1 & 0 \\ 0 & 0\end{array}\right)$. Starting with two initially circular voids, the presence of elastic stresses leads to a vertical split in the material; see Figure 8. We used the following parameters for $\left(\widetilde{\mathrm{P}}_{\varepsilon}^{h, \tau}\right): \gamma=\frac{1}{24 \pi}, T=5 \times 10^{-5}$ and $\tau_{n}=\tau=10^{-7}$. As initial data we chose (4.2) $(i i)$ with $z=-\tilde{z}=(0,0.23), R=\widetilde{R}=0.18$. The refinement parameters were $N_{f}=256$ and $N_{c}=32$.

Further results, including simulations modelling the (combined) effect of surface diffusion, an electric field, grain boundaries and anisotropic elasticity will be reported on elsewhere (see [9]), where we also discuss applications to epitaxial growth.

\section{ACKNowledgments}

Part of this work was carried out while the authors participated in the 2003 programme Computational Challenges in Partial Differential Equations at the Isaac Newton Institute, Cambridge, UK. This work was also supported by the RTN Programme Fronts-Singularities, HPRN-CT-2002-00274 and the DFG Priority Program Analysis, Modeling and Simulation of Multiscale Problems. 


\section{REFERENCES}

1. R. A. Adams and J. Fournier, Cone conditions and properties of Sobolev spaces, J. Math. Anal. Appl. 61 (1977), 713-734. MR0463902(57:3840)

2. R. J. Asaro and W. A. Tiller, Interface morphology development during stress corrosion cracking: Part i. via surface diffusion, Metall. Trans. 3 (1972), 1789-1796.

3. J. W. Barrett and J. F. Blowey, Finite element approximation of a degenerate AllenCahn/Cahn-Hilliard system, SIAM J. Numer. Anal. 39 (2001), 1598-1624. MR1885708 (2003a:65078)

4. J. W. Barrett, J. F. Blowey, and H. Garcke, Finite element approximation of a fourth order nonlinear degenerate parabolic equation, Numer. Math. 80 (1998), 525-556. MR1650035 (99j:65144)

5. , Finite element approximation of the Cahn-Hilliard equation with degenerate mobility, SIAM J. Numer. Anal. 37 (1999), 286-318. MR1742748 (2001c:65118)

6. - On fully practical finite element approximations of degenerate Cahn-Hilliard systems, M2AN Math. Model. Numer. Anal. 35 (2001), 713-748. MR.1863277 (2002k:65149)

7. J. W. Barrett, H. Garcke, and R. Nürnberg, Finite element approximation of surfactant spreading on a thin film, SIAM J. Numer. Anal. 41 (2003), 1427-1464. MR.2034888 (2005a:65098)

8. _ A phase field model for electromigration of intergranular voids, 2005, (submitted for publication).

9. — Phase field models for stress and electromigration induced surface diffusion with applications to epitaxial growth and void evolution, 2005, (in preparation).

10. J. W. Barrett, S. Langdon, and R. Nürnberg, Finite element approximation of a sixth order nonlinear degenerate parabolic equation, Numer. Math. 96 (2004), 401-434. MR.2028722 (2004k:65168)

11. J. W. Barrett and R. Nürnberg, Convergence of a finite element approximation of surfactant spreading on a thin film in the presence of van der Waals forces, IMA J. Numer. Anal. 24 (2004), 323-363. MR2046180(2005a:65099)

12. J. W. Barrett, R. Nürnberg, and V. Styles, Finite element approximation of a phase field model for void electromigration, SIAM J. Numer. Anal. 42 (2004), 738-772. MR2084234

13. J. Bergh and J. Löfström, Interpolation spaces, an introduction, Springer, Berlin, 1976. MR 0482275 (58:2349)

14. D. N. Bhate, A. Kumar, and A. F. Bower, Diffuse interface model for electromigration and stress voiding, J. Appl. Phys. 87 (2000), 1712-1721.

15. J. F. Blowey and C. M. Elliott, The Cahn-Hilliard gradient theory for phase separation with non-smooth free energy. Part I: Mathematical analysis, European J. Appl. Math. 2 (1991), 233-279. MR1123143 (93a:35025)

16. A. F. Bower and D. Craft, Analysis of failure mechanisms in the interconnect lines of microelectronic circuits, Fat. Frac. Eng. Mat. Struct. 21 (1998), 611-630.

17. S. C. Brenner and L. R. Scott, The mathematical theory of finite element methods (second edition), Springer, New York, 2002. MR.1894376 (2003a:65103)

18. J. W. Cahn, C. M. Elliott, and A. Novick-Cohen, The Cahn-Hilliard equation with a concentration dependent mobility: motion by minus the Laplacian of the mean curvature, European J. Appl. Math. 7 (1996), 287-301. MR 1401172 (97g:80010)

19. M. Carrive, A. Miranville, and A. Piétrus, The Cahn-Hilliard equation for deformable elastic media, Adv. Math. Sci. Appl. 10 (2000), 539-569. MR1807441 (2001m:35295)

20. M. Costabel, M. Dauge, and Y. Lafranche, Fast semi-analytic computation of elastic edge singularities, Comp. Meth. Appl. Mech. Eng. 190 (2001), 2111-2134.

21. C. M. Elliott, Approximation of curvature dependent interface motion, The State of the Art in Numerical Analysis (New York) (I. S. Duff and G. A. Watson, eds.), Oxford University Press, 1997, pp. 407-440. MR.1628355 (99c:35120)

22. C. M. Elliott and H. Garcke, On the Cahn-Hilliard equation with degenerate mobility, SIAM J. Math. Anal. 27 (1996), 404-423. MR.1377481 (97c:35081)

23. $465-488$.

24. J. Escher, U. F. Mayer, and G. Simonett, The surface diffusion flow for immersed hypersurfaces, SIAM J. Math. Anal. 29 (1998), 1419-1433. MR.1638074 (99f:58042) 
25. E. Fried and M. E. Gurtin, Dynamic solid-solid transitions with phase characterized by an order parameter, Physica D 72 (1994), 287-308. MR1271571 (95g:73007)

26. H. Garcke, On mathematical models for phase separation in elastically stressed solids, 2000, Habilitation, University Bonn.

27. 307-331. MR1969816 (2004k:74080)

28. _ On a Cahn-Hilliard model for phase separation with elastic misfit, Ann. Inst. H. Poincaré Anal. Non Linéaire 22 (2005), 165-185. MR2124161

29. H. Garcke, M. Rumpf, and U. Weikard, The Cahn-Hilliard equation with elasticity - finite element approximation and qualitative studies, Interfaces Free Bound. 3 (2001), 101-118. MR $1805080(2001 \mathrm{k}: 65149)$

30. H. Garcke and U. Weikard, Numerical approximation of the Cahn-Larché equation, Numer. Math. (2005), (to appear).

31. M. Giaquinta and G. Modica, Regularity results for some classes of higher order nonlinear elliptic systems, J. Reine Angew. Math. 311-312 (1979), 145-169. MR0549962 (81a:35035)

32. M. A. Grinfeld, Instability of the separation boundary between a non-hydrostatically stressed elastic body and a melt, Soviet Phys. Dokl. 31 (1986), 831-834.

33. P. Grisvard, Singularités en elasticité, Arch. Ration. Mech. Anal. 107 (1989), 157-180. MR0996909 (90j:35170)

34. G. Grün, On the convergence of entropy consistent schemes for lubrication type equations in multiple space dimensions, Math. Comp. 72 (2003), 1251-1279. MR1972735(2004c:65109)

35. G. Grün and M. Rumpf, Nonnegativity preserving numerical schemes for the thin film equation, Numer. Math. 87 (2000), 113-152. MR1800156 (2002h:76108)

36. M. R. Gungor, D. Maroudas, and L. J. Gray, Effects of mechanical stress on electromigrationdriven transgranular void dynamics in passivated metallic thin films, Appl. Phys. Lett. 73 (1998), 3848-3850.

37. M. E. Gurtin, The linear theory of elasticity, Handbuch der Physik, Vol. VIa/2 (S. Flügge and C. Truesdell, eds.), Springer, Berlin, 1972.

38. K. Kassner, C. Misbah, J. Müller, J. Kappey, and P. Kohlert, Phase-field modeling of stressinduced instabilities, Phys. Rev. E 63 (2001), 036117 (27 pages).

39. P. H. Leo, J. S. Lowengrub, and H. J. Jou, A diffuse interface model for microstructural evolution in elastically stressed solids, Acta Mater. 46 (1998), 2113-2130.

40. P. P. Mosolov and V. P. Mjasnikov, A proof of Korn's inequality, Soviet Math. Dokl. 12 (1971), 1618-1622. MR0296685 (45:5744)

41. J. Nečas and I. Hlaváček, Mathematical theory of elastic and elasto-plastic bodies: An introduction, Elsevier, Amsterdam, 1981. MR0600655 (82h:73002)

42. R. Rannacher and R. Scott, Some optimal error estimates for piecewise linear finite element approximations, Math. Comp. 38 (1982), 437-445. MR0645661(83e:65180)

43. A. Schmidt and K. G. Siebert, ALBERT - software for scientific computations and applications, Acta Math. Univ. Comenian. 70 (2000), 105-122. MR1865363

44. J. B. Seif, On the Green's function for the biharmonic equation in an infinite wedge, Trans. Amer. Math. Soc. 182 (1973), 241-260. MR0325989(48:4335)

45. B. J. Spencer, P. W. Voorhees, and S. H. Davis, Morphological instability in epitaxially strained dislocation-free solid films: Linear stability theory, J. Appl. Phys. 73 (1993), 4955-4970.

46. L. Xia, A. F. Bower, Z. Suo, and C. Shih, A finite element analysis of the motion and evolution of voids due to strain and electromigration induced surface diffusion, J. Mech. Phys. Solids 45 (1997), 1473-1493.

47. L. Zhornitskaya and A. L. Bertozzi, Positivity preserving numerical schemes for lubricationtype equations, SIAM J. Numer. Anal. 37 (2000), 523-555. MR.1740768(2000m:65100)

Department of Mathematics, Imperial College, London, SW7 2AZ, United Kingdom E-mail address: j.barrett@imperial.ac.uk

NWF I - Mathematik, Universität Regensburg, 93040 Regensburg, Germany

E-mail address: harald.garke@mathematik. uni-regensburg.de

Department of Mathematics, Imperial College, London, SW7 2AZ, United Kingdom

E-mail address: robert.nurnberg@imperial.ac.uk 